\title{
Southern Appalachian \\ Regional Seismic Network
}

Manuscript Completed: June 1994

Date Published: August 1994

Prepared by

S-C. C. Chiu, A. C. Johnston, J-M. Chiu

Center for Earthquake Research and Information Memphis State University

Memphis, TN 38152

Prepared for

Division of Engineering

Office of Nuclear Regulatory Research

U.S. Nuclear Regulatory Commission

Washington, DC 20555-0001

NRC FIN D1553 


\section{DISCLAIMER}

This report was prepared as an account of work sponsored by an agency of the United States Government. Neither the United States Government nor any agency Thereof, nor any of their employees, makes any warranty, express or implied, or assumes any legal liability or responsibility for the accuracy, completeness, or usefulness of any information, apparatus, product, or process disclosed, or represents that its use would not infringe privately owned rights. Reference herein to any specific commercial product, process, or service by trade name, trademark, manufacturer, or otherwise does not necessarily constitute or imply its endorsement, recommendation, or favoring by the United States Government or any agency thereof. The views and opinions of authors expressed herein do not necessarily state or reflect those of the United States Government or any agency thereof. 


\section{DISCLAIMER}

Portions of this document may be illegible in electronic image products. Images are produced from the best available original document. 


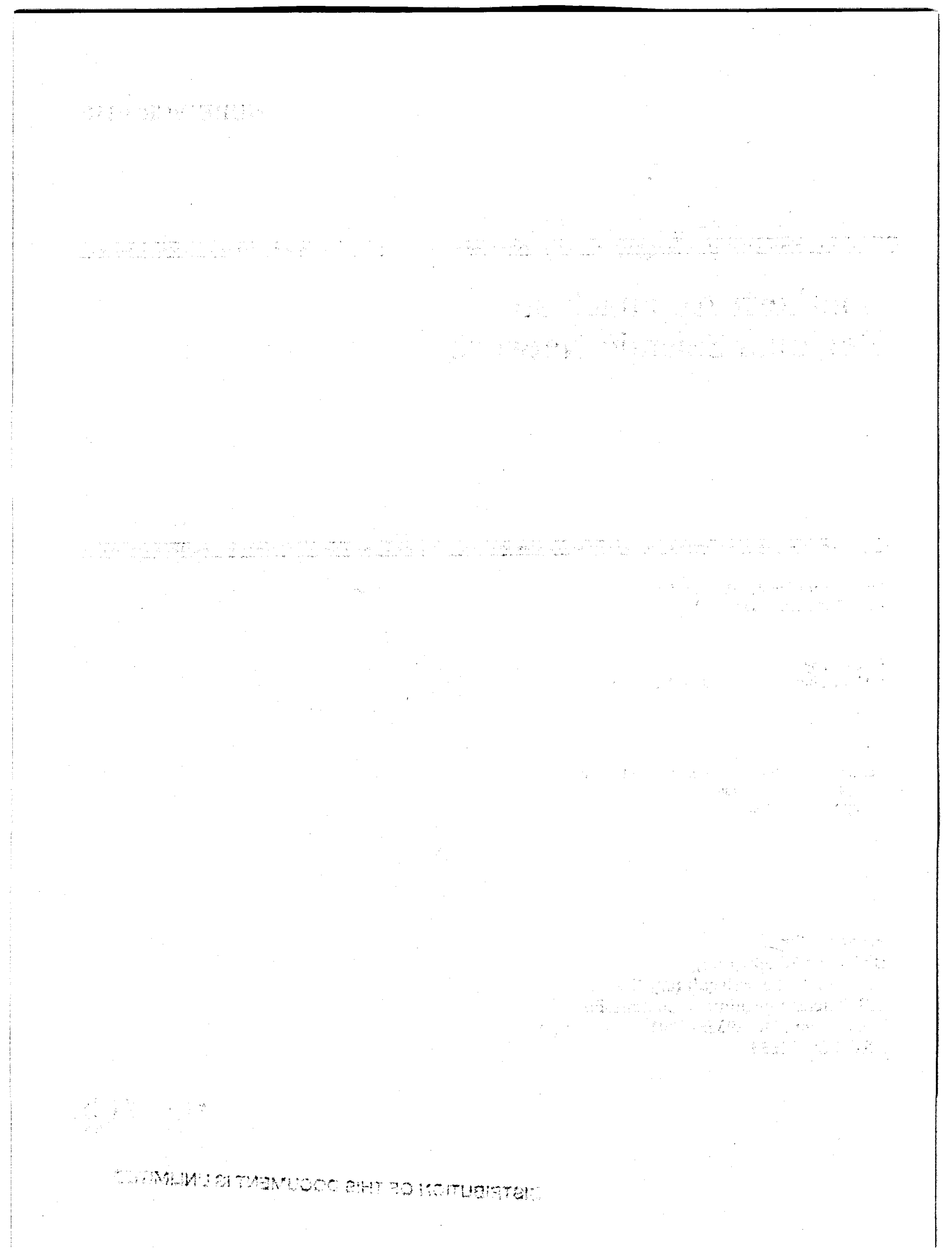




\begin{abstract}
The seismic activity in the southern Appalachian area was monitored by the Southern Appalachian Regional Seismic Network (SARSN) since late 1979 by the Center for Earthquake Research and Information (CERI) at Memphis State University. This network provides good spatial coverage for earthquake locations especially in east Tennessee. The level of activity concentrates more heavily in the Valley and Ridge province of eastern Tennessee, as opposed to the Blue Ridge or Inner Piedmont. The large majority of these events lie between New York - Alabama lineament and the Clingman/Ocoee lineament, magnetic anomalies produced by deep-seated basement structures. Therefore SARSN, even with its wide station spacing, has been able to define the essential first-order seismological characteristics of the Southern Appalachian seismic zone.

The focal depths of the southeastern U. S. earthquakes concentrate between 8 and 16 km, occurring principally beneath the Appalachian overthrust. In cross-sectional views, the average seismicity is shallower to the east beneath the Blue Ridge and Piedmont provinces and deeper to the west beneath the Valley and Ridge and the North American craton.

Results of recent focal mechanism studies by using the CERI digital earthquake catalog between October, 1986 and December, 1991, indicate that the basement of the Valley and Ridge province is under a horizontal, NE-SW compressive stress. Right-lateral strike-slip faulting on nearly north-south fault planes is preferred because it agrees with the trend of the regional magnetic anomaly pattern.
\end{abstract}


Table of Contents

List of Figures

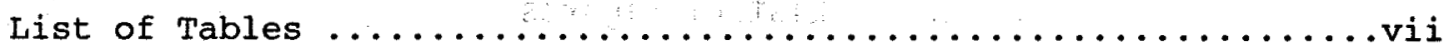

Introduction $\ldots \ldots \ldots \ldots \ldots \ldots \ldots \ldots \ldots \ldots \ldots \ldots \ldots \ldots \ldots \ldots$

Seismic Network Configuration $\ldots \ldots \ldots \ldots \ldots \ldots \ldots \ldots \ldots \ldots \ldots$

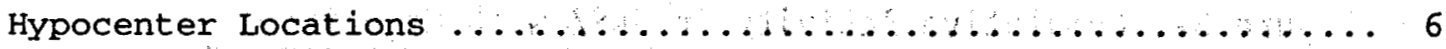

Crustal velocity model $\ldots \ldots \ldots \ldots \ldots \ldots \ldots \ldots \ldots \ldots \ldots \ldots \ldots \ldots$

Seismicity in the $\operatorname{sasz} \ldots \ldots \ldots \ldots \ldots \ldots \ldots \ldots \ldots \ldots \ldots \ldots \ldots \ldots \ldots 7$

Active Faults in Eastern Tennessee $\ldots \ldots \ldots \ldots \ldots \ldots \ldots \ldots \ldots \ldots$

Focal Mechanism Study in Eastern Tennessee $\ldots \ldots \ldots \ldots \ldots \ldots \ldots \ldots 16$

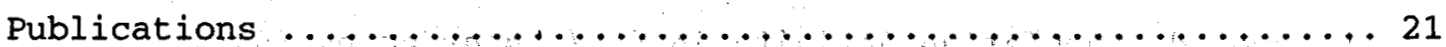

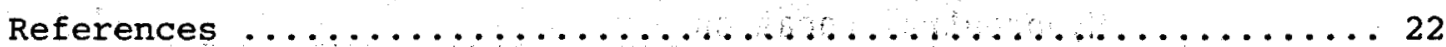

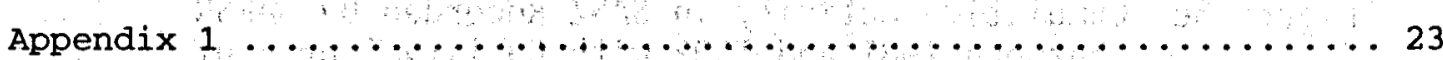




\section{List of Figures}

Figure 1. SARSN Station Map

Figure 2. Seismicity in South Central U.S.A. Recorded by SARSN and MARSN between 1974 and 1992

Figure 3a. Cumulative Activity in SASZ with Magnitude

Greater Than or Equal To 0.0 and Focal Depth

Greater Than or Equal To $5.0 \mathrm{~km}$ Recorded

by SARSN between 1981 and 1992

Figure 3b. Cumulative Activity in SASZ with Magnitude Greater Than or Equal To 3.0 Recorded by SARSN between 1981 and 1992

Figure 3c. Cumulative Activity in SASZ Recorded by SARSN between 1981 and 1992 with Quality A of Hypocentral Location

Figure 3d. Cumulative Activity in SASZ Recorded by SARSN between 1981 and 1992 with Quality B of Hypocentral Location

Figure 3e. Cumulative Activity in SASZ Recorded by SARSN between 1981 and 1992 with Quality A or B of Hypocentral Location

Figure 4a. Depth Distribution of the Analog Earthquake Catalog Between September, 1981 and September, 1986

Figure 4b. Depth Distribution of the Digital Earthquake Catalog between October, 1986 and December, 1991

Figure 5a. Index Map for the Cross-sectional Views in SASZ 15

Figure 5b. Seven Cross-sections of Depth Distribution in SASZ

Figure 6. Locations of 19 Selected Events and Their Focal Mechanisms 
List of Tables

Table 1 Information on SARSN stations $\ldots \ldots \ldots \ldots \ldots \ldots \ldots \ldots$

Table 2 Summary of 19 selected Focal Mechanisms $\ldots \ldots \ldots \ldots \ldots \ldots$ 


\section{INTRODUCTION}

Under U.S. Nuclear Regulatory Commission grant support (original grant NRC-04-78200 followed by NRC-04-85-107), the Southern Appalachian Regional Seismic Network was established in late 1979 by the Center for Earthquake Research and Information (CERI) at Memphis State University to provide supplemental regional seismic monitoring in eastern Tennessee. The first stations were installed in early 1981, a locational ability was achieved in mid-1981, and the network was completed in early 1983. Earthquake data collected by SARSN and by the Tennessee Valley Authority (TVA) have become the primary source for the assessment of regional seismic hazard and more advanced seismological, geological, structural, and tectonic research in the southern Appalachian seismic zone (SASZ). The CERI Quarterly Seismological Bulletin which combined data from SARSN and the Memphis Area Regional Seismic Network(MARSN) was published routinely by the Center for Earthquake Research and Information. Figure 1 shows station locations for SARSN. A map of epicenters located by this regional network is shown in the Figure 3a. The distribution pattern of earthquake locations in the SASZ clearly indicates a heavy concentration of activity in the Valley and Ridge province of east Tennessee.

\section{SEISMIC NETWORK CONFIGURATION}

The southern Appalachian study area is the region between $81^{\circ}$ to $87^{\circ} \mathrm{W}$ longitude and $34.5^{\circ}$ to $37^{\circ} \mathrm{N}$ latitude. As such, it includes the Valley and Ridge and Blue Ridge physiographic provinces, portions of the Inner Piedmont and Charlotte Belt to the east, and the Cumberland Plateau and Nashville Dome to the West (Figure 2). This network has a station density of $50-130 \mathrm{~km}$ in the southern Appalachians of eastern Tennessee and western North Carolina, thus obviating reliable determination of hypocentral depth on a routine basis. However, increased station density, through the joint efforts of CERI, Georgia Institute of Technology, which operated a five-station array in southeastern Tennessee from April, 1982 to recent, and the Tennessee Valley Authority's 18 station network, results in good depth control in some portions of SASZ.

Figure 1 shows the location distribution of SARSN stations. SARSN consists of 18 short period seismic stations that cover parts of 4 states: eastern Tennessee, western North Carolina, southwestern Virginia, and northern Georgia. Signals are telemetered via radio to central receiving sites in Knoxville, Tennessee and Morganton, North Carolina and multiplexed for leased telephone transmission to CERI in Memphis. All but 3 of the 18 permanent seismic stations have vertical single component sensors, and all but a few

have modest dynamic range $(40-60 \mathrm{~dB})$. Table 1 contains information on the station code, location, instrumentation and calibration, operation status, and site geology of the CERI SARSN stations. All short-period data (and long-period data for one station) have been continuously recorded digitally at 50 samples per second on a PDP 11/41 since late 1986. Selected stations are also recorded in analog form on pen-and-ink paper records. Prior to November 11, 1989, analog data were also recorded on 16-mm Develocorder film. Arrival times are read from digital records to an accuracy of 0.01 seconds and from pen-and-ink records to an accuracy of 0.1 seconds. 


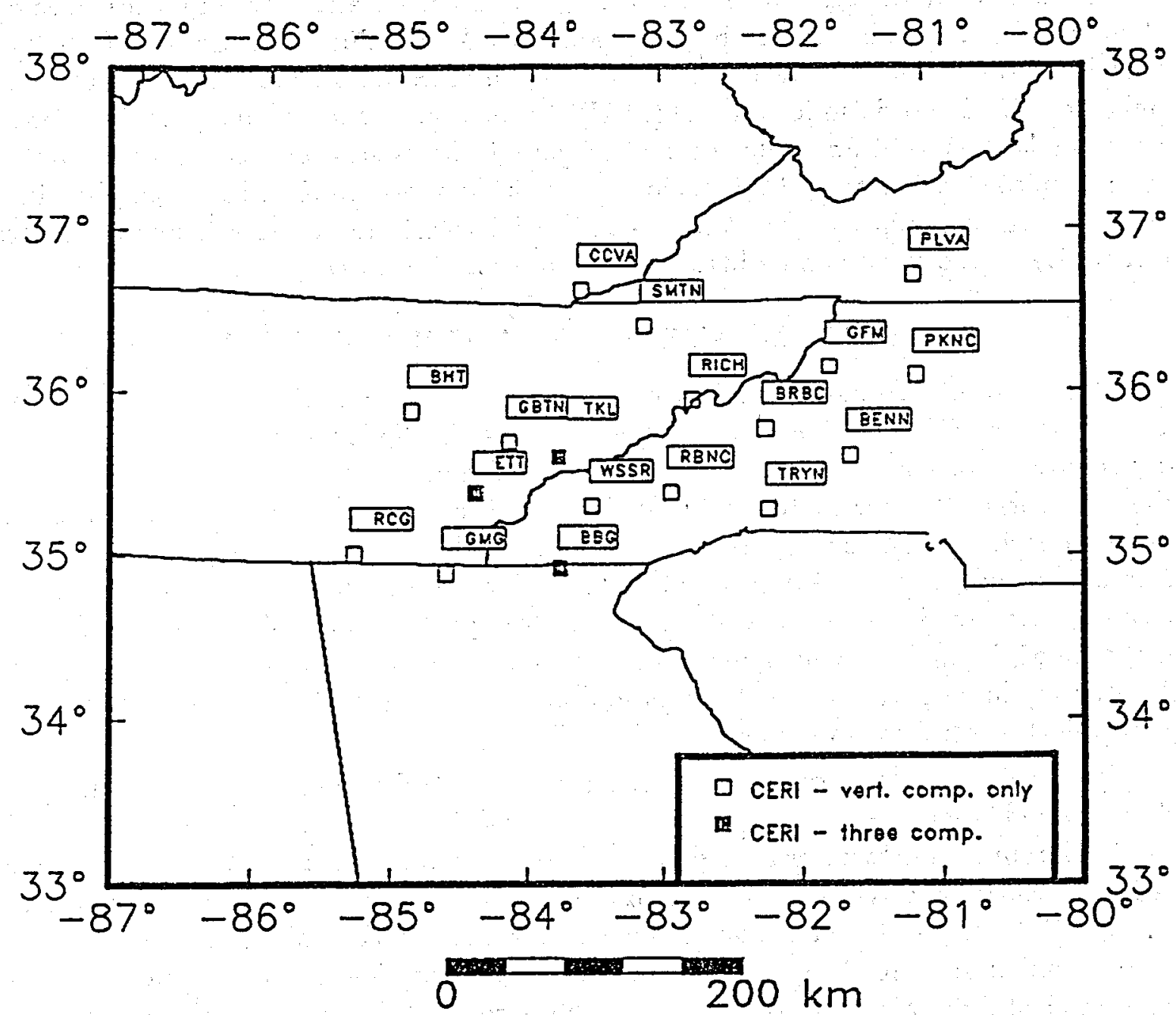

Figure 1. Southern Appalachian Regional Seismic Network. Solid squares denote 3component stations. 


\section{Southcentral United States Seismicity: 1974 - 1992}

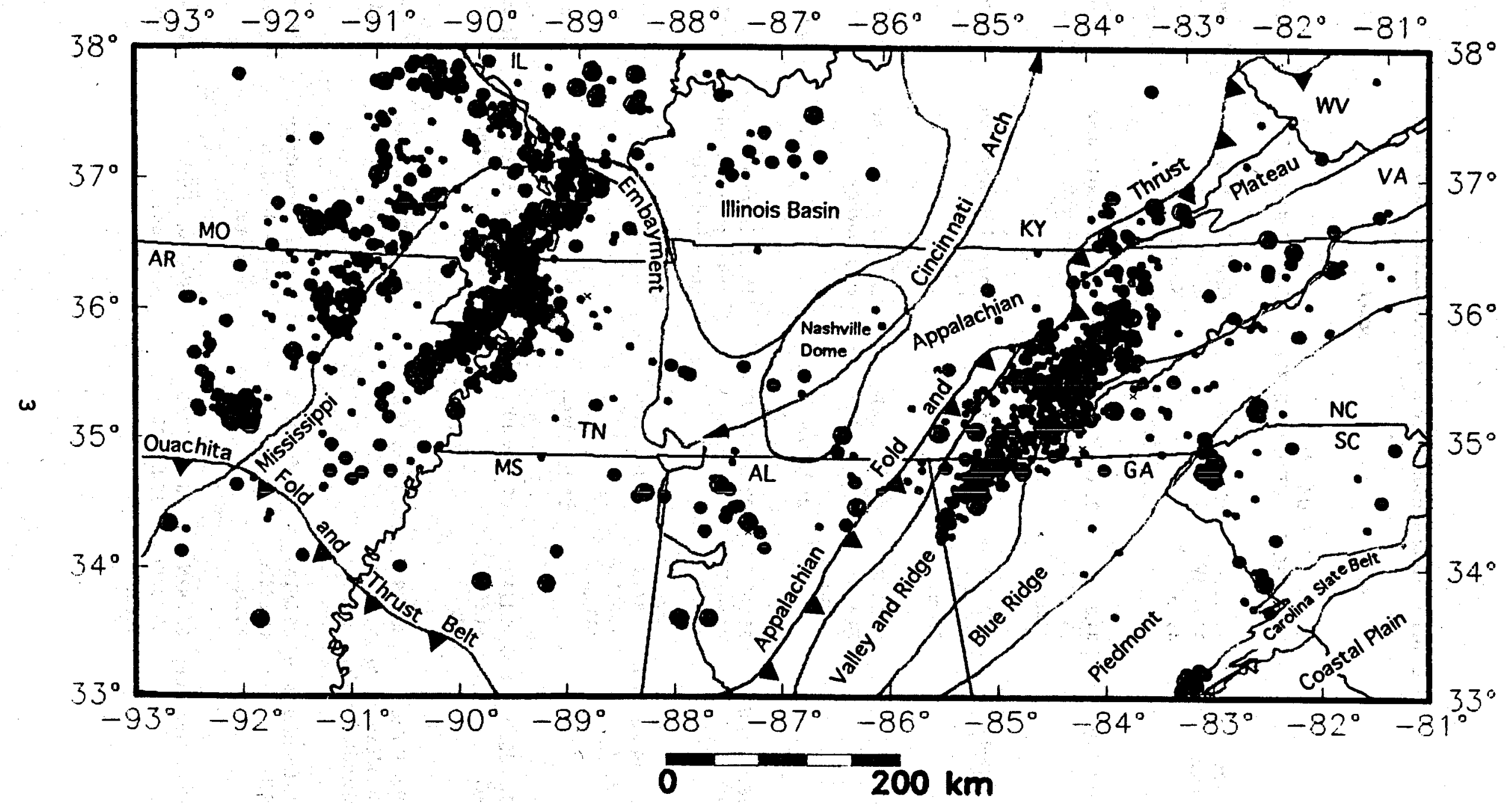

Figure 2. Earthquake activity in the south central United States recorded by SARSN and MARSN between 1974 and 1992. Geological provinces are separated and indicated by lines. Dots represent the epicenters. Magnitude code is as follows:
$4.0 \leq M<5.0$
- $3.0 \leqq M<4.0$;
- $2.0 \leqq M<3.0$;
- $0.0 \leqq M<2.0$;
$\times \quad M<0.0$. 
Table 1: Information on Permanent CERT Stations

SARSN

\begin{tabular}{|c|c|c|c|c|c|}
\hline CODE & STATION & LOCATION & $\begin{array}{l}\text { DATE } \\
\text { OPENED/CLOSED }\end{array}$ & SITE GEOLOGY & INSTRUMENTATION \\
\hline TKL & $\begin{array}{l}\text { Tuckaleechee } \\
\text { Caverns, TN }\end{array}$ & $\begin{array}{l}35.658 \mathrm{~N} \\
83.774 \mathrm{~W} \\
351 \mathrm{~m}\end{array}$ & $\begin{array}{l}30 \text { Jul 1978- } \\
24 \text { Jul 1985- }\end{array}$ & $\begin{array}{l}\text { Cambrian-Ordovician } \\
\text { limestone } \\
\text { Knox Group }\end{array}$ & $\begin{array}{l}\text { Geotech } S-13 \\
T_{o}=1 \mathrm{sec} \\
\text { horizontals: } T_{o}=1 \mathrm{sec}\end{array}$ \\
\hline RCG & Rock City, GA & $\begin{array}{l}34.975 \mathrm{~N} \\
85.348 \mathrm{~W} \\
468 \mathrm{~m}\end{array}$ & 23 Oct 1981- & $\begin{array}{l}\text { Lower Pennsylvanian } \\
\text { conglomerate }\end{array}$ & $\begin{array}{l}\text { Geotech } S-13 \\
T_{0}=1 \mathrm{sec}\end{array}$ \\
\hline BHT & Blowhole, TN & $\begin{array}{l}35.847 \mathrm{~N} \\
84.945 \mathrm{~W} \\
826 \mathrm{~m}\end{array}$ & 23 Oct 1981- & $\begin{array}{l}\text { Lower Pennslyvanian } \\
\text { sandstone }\end{array}$ & $\begin{array}{l}\text { Geotech S-13 } \\
T_{o}=1 \mathrm{sec}\end{array}$ \\
\hline ETT & Etowah, TN & $\begin{array}{l}35.326 \mathrm{~N} \\
84.455 \mathrm{~W} \\
588 \mathrm{~m}\end{array}$ & $\begin{array}{l}25 \text { Nov 1981- } \\
21 \text { Jul 1985- }\end{array}$ & $\begin{array}{l}\text { Lower Pennslyvanian } \\
\text { conglomerate }\end{array}$ & $\begin{array}{l}\text { Geotech S-13 } \\
T_{o}=1 \mathrm{sec} \\
\text { horizontals; } T_{o}=1 \mathrm{se}\end{array}$ \\
\hline BENN & $\begin{array}{l}\text { Benn Knob, } \\
\text { NC }\end{array}$ & $\begin{array}{l}35.565 \mathrm{~N} \\
81.661 \mathrm{~W} \\
878 \mathrm{~m}\end{array}$ & 22 Jan 1982- & $\begin{array}{l}\text { Upper Precambrian } \\
\text { metamorphic complex } \\
\text { gneiss and schist }\end{array}$ & $\begin{array}{l}\text { Geotech S-13 } \\
T_{o}=1 \mathrm{sec}\end{array}$ \\
\hline BBG & $\begin{array}{l}\text { Brasstown Bald, } \\
\text { GA }\end{array}$ & $\begin{array}{l}34.874 \mathrm{~N} \\
83.811 \mathrm{~W} \\
1355 \mathrm{~m}\end{array}$ & 27 Jan 1982- & $\begin{array}{l}\text { Upper Precambrian } \\
\text { schist }\end{array}$ & $\begin{array}{l}\text { Geotech S-13 } \\
T_{o}=1 \mathrm{sec} \\
\text { horizontals; } T_{o}=1 \mathrm{~s}\end{array}$ \\
\hline GFM & $\begin{array}{l}\text { Grandfather Mtn, } \\
\text { NC }\end{array}$ & $\begin{array}{l}36.111 \mathrm{~N} \\
81.807 \mathrm{~W} \\
1726 \mathrm{~m}\end{array}$ & 27 May 1982- & $\begin{array}{l}\text { Upper Precambrian } \\
\text { metamorphic complex }\end{array}$ & $\begin{array}{l}\text { Geotech S-13 } \\
T_{o}=1 \mathrm{sec}\end{array}$ \\
\hline BRBC & $\begin{array}{l}\text { Blue Ridge } \\
\text { Broadcasting Co. } \\
\text { Mt Mitchell, NC }\end{array}$ & $\begin{array}{l}35.739 \mathrm{~N} \\
82.286 \mathrm{~W} \\
1976 \mathrm{~m}\end{array}$ & 26 May 1982- & $\begin{array}{l}\text { Upper Precambrian } \\
\text { metasedimentary } \\
\text { sequence }\end{array}$ & $\begin{array}{c}\text { Geotech S-13 } \\
T_{o}=1 \text { sec }\end{array}$ \\
\hline RBNC & $\begin{array}{l}\text { Richland Balsam, } \\
\text { NC }\end{array}$ & $\begin{array}{l}35.357 \mathrm{~N} \\
82.986 \mathrm{~W} \\
1829 \mathrm{~m}\end{array}$ & 26 May 1982- & $\begin{array}{l}\text { Upper Precambrian } \\
\text { metasedimentary } \\
\text { sequence. }\end{array}$ & $\begin{array}{l}\text { Geotech S-13 } \\
T_{0}=1 \mathrm{sec}\end{array}$ \\
\hline SMTN & $\begin{array}{l}\text { Short Mountain, } \\
\text { TN }\end{array}$ & $\begin{array}{l}36.382 \mathrm{~N} \\
83.182 \mathrm{~W} \\
768 \mathrm{~m}\end{array}$ & $\begin{array}{l}\text { 05 Oct } 1982- \\
19 \text { Dec } 1991 \\
\text { closed }\end{array}$ & $\begin{array}{l}\text { Silurian Clinch } \\
\text { sandstone }\end{array}$ & $\begin{array}{l}\text { Geotech S-13 } \\
T_{o}=1 \mathrm{sec}\end{array}$ \\
\hline
\end{tabular}


Table 1 Continued

\begin{tabular}{|c|c|c|c|c|c|}
\hline CODE & STATION & LOCATION & $\begin{array}{l}\text { DATE } \\
\text { OPENED/CLOSED }\end{array}$ & SITE GEOLOGY & INSTRUMENTATION \\
\hline FGTN & $\begin{array}{l}\text { Flat Gap, } \\
\text { TN }\end{array}$ & $\begin{array}{l}36.434 \mathrm{~N} \\
83.195 \mathrm{~W} \\
499.87 \mathrm{~m}\end{array}$ & 19 Dec 1991- & $\begin{array}{l}\text { Mississippian } \\
\text { limestone }\end{array}$ & $\begin{array}{l}\text { Geotech } \mathrm{S}-13 \\
T_{0}=1 \mathrm{sec}\end{array}$ \\
\hline CCVA & $\begin{array}{l}\text { Cudjo Caverns, } \\
\text { VA }\end{array}$ & $\begin{array}{l}36.602 \mathrm{~N} \\
83.667 \mathrm{~W} \\
509 \mathrm{~m} \\
36.603 \mathrm{~N} \\
83.667 \mathrm{~W} \\
571 \mathrm{~m}\end{array}$ & $\begin{array}{l}06 \text { Oct } 1982- \\
12 \text { Nov } 1982 \\
\text { closed } \\
12 \text { Nov } 1982-\end{array}$ & $\begin{array}{l}\text { Mississippian } \\
\text { Newman } \\
\text { limestone }\end{array}$ & $\begin{array}{l}\text { Geotech S-13 } \\
T_{0}=1 \mathrm{sec}\end{array}$ \\
\hline PKNC & $\begin{array}{l}\text { Pores Knob, } \\
\text { NC }\end{array}$ & $\begin{array}{l}36.046 \mathrm{~N} \\
81.158 \mathrm{~W} \\
785 \mathrm{~m}\end{array}$ & 11 Nov $1882-$ & $\begin{array}{l}\text { Devonian } \\
\text { Heldenberg Group } \\
\text { limestone }\end{array}$ & $\begin{array}{l}\text { Geotech S-13 } \\
T_{o}=1 \mathrm{sec}\end{array}$ \\
\hline PLVA & $\begin{array}{l}\text { Point Lookout, } \\
\text { VA }\end{array}$ & $\begin{array}{l}36.666 \mathrm{~N} \\
81.161 \mathrm{~W} \\
1353 \mathrm{~m}\end{array}$ & 30 Nov 1982 & $\begin{array}{l}\text { Precambrian } \\
\text { Striped Rock } \\
\text { granite }\end{array}$ & $\begin{array}{l}\text { Geotech S-13 } \\
T_{o}=1 \mathrm{sec}\end{array}$ \\
\hline TRYN & $\begin{array}{l}\text { Tryon Peak, } \\
\text { NC } \\
\end{array}$ & $\begin{array}{l}35.267 \mathrm{~N} \\
82.244 \mathrm{~W} \\
968 \mathrm{~m} \\
35.246 \mathrm{~N} \\
82.267 \mathrm{~W} \\
915 \mathrm{~m}\end{array}$ & $\begin{array}{l}30 \text { Nov } 1982- \\
22 \text { Mar } 1983 \\
22 \text { Mar } 1983-\end{array}$ & $\begin{array}{l}\text { Precambrian } \\
\text { metamorphic } \\
\text { gneiss }\end{array}$ & $\begin{array}{l}\text { Geotech S-13 } \\
T_{0}=1 \mathrm{sec}\end{array}$ \\
\hline GBTN & Greenback, TN & $\begin{array}{l}35.666 \mathrm{~N} \\
84.211 \mathrm{~W} \\
326 \mathrm{~m}\end{array}$ & 23 Mar $1983-$ & $\begin{array}{l}\text { Ordovician Holston } \\
\text { limestone }\end{array}$ & $\begin{array}{l}\text { Geotech S-13 } \\
T_{0}=1 \mathrm{sec}\end{array}$ \\
\hline RICH & Rich Mtn, NC & $\begin{array}{l}35.920 \mathrm{~N} \\
82.820 \mathrm{~W} \\
968 \mathrm{~m}\end{array}$ & 18 Jun $1983-$ & $\begin{array}{l}\text { Upper Precambrian } \\
\text { metasedimentary } \\
\text { sequence }\end{array}$ & $\begin{array}{l}\text { Geotech } S-13 \\
T_{o}=1 \mathrm{sec}\end{array}$ \\
\hline GMG & $\begin{array}{l}\text { Grassy Mtn, } \\
\text { GA }\end{array}$ & $\begin{array}{l}34.863 \mathrm{~N} \\
84.670 \mathrm{~W} \\
1097 \mathrm{~m}\end{array}$ & 27 Sep 1985- & Quarte sandstone & $\begin{array}{l}\text { Geotech S-13 } \\
T_{0}=1 \mathrm{sec}\end{array}$ \\
\hline WSSR & $\begin{array}{l}\text { Wesser Bald, } \\
\text { NC }\end{array}$ & $\begin{array}{l}35.278 \mathrm{~N} \\
83.578 \mathrm{~W} \\
1340 \mathrm{~m}\end{array}$ & 04 Oct 1985 & $\begin{array}{l}\text { Gneiss/ mica } \\
\text { schist }\end{array}$ & $\begin{array}{l}\text { Geotech S-13 } \\
T_{o}=1 \mathrm{sec}\end{array}$ \\
\hline
\end{tabular}




\section{HYPOCENTER LOCATIONS}

All earthquakes recorded by a sufficient number of SARSN stations have been located using either HYPO71 (Lee and Lahr, 1975) or HYPOELLIPSE (Lahr, 1984). HYPO71 was used to locate events recorded by the SARSN network from September 1981 through December 1985 and HYPOELLIPSE has been used since then. CERI readings are supplemented by other regional data whenever possible. An earthquake catalog from September, 1982 through December, 1992 is listed in Appendix 1. Events within the area of $81.0^{\circ}-87.0^{\circ} \mathrm{W}$ longitude and $34.5^{\circ}-37.0^{\circ} \mathrm{N}$ latitude are located by SARSN and published in Table II(A) in the CERI Quarterly Seismological Bulletin. Other regional earthquakes hypocenters are published in Table II(B) of the Quarterly Bulletin for informational purposes only. Earthquakes are located to an epicentral accuracy of 1-2 km; in general, depths are uncertain by $5 \mathrm{~km}$ or more unless close-in station control exists. In the case of poor azimuthal station distribution or lack of depth control, the depth is restricted to a value yielding the smallest travel time residual (RMS).

In Appendix 1, several quantities can be used to judge the general quality of the hypocenter solution. ST/PH indicates the number of stations and phases actually used in the hypocenter computation. DMIN is the epicentral distance in kilometers to the closest station. GAP is the maximum arc sector in degrees of azimuth between recording stations. RMS is the root-mean-square in seconds of the travel-time residuals. SQD indicates HYPOELLIPSE two solution quality grades, the first based on travel-time residuals of recorded phases, and the second on station distribution. $Q$, is an overall measure of solution quality based on an average of these two factors. ERH1, ERH2, ERZ, AZM are computed error measures as specified in HYPOELLIPSE. ERH1 and ERH2 are the semi-major and semi-minor axes of the horizontal error ellipse, respectively, and ERZ is the vertically projected semi-major axis of the error ellipsoid. AZM is the bearing, with respect to north, of the semi-major axis (ERH1).

\section{CRUSTAL VELOCITY MODEL}

The crustal velocity model used for the SARSN region is given as the Moore model (MOR) that was developed by VPI and SU (Moore, 1980) for Giles County located in the Virginia Valley and Ridge province. The MOR model has been extensively tested and shown appropriate to the southern Appalachians (Bollinger et al., 1980; Johnston, 1985). It is summarized below:

$\begin{array}{lll}\text { Model } & \begin{array}{l}\text { Depth } \\ (\mathrm{km})\end{array} & \begin{array}{l}\text { P-Velocity } \\ (\mathrm{km} / \mathrm{sec})\end{array} \\ \text { Moore } & & \\ & 0.0-5.7 & 5.63 \\ & 5.7-14.7 & 6.05 \\ & 14.7-50.7 & 6.53 \\ & \text { Halfspace } & 8.18\end{array}$


S-Velocities corresponding to each $\mathrm{P}$-velocity may be calculated by:

$$
V_{s}=V_{p} / \sqrt{3}
$$

SARSN magnitudes are computed using duration formulae developed by others for the eastern United States:

$$
M_{d}=2.23 \log (D)+0.12 \log (k)-2.36
$$

where $D$ is the coda duration in seconds and $k$ is the epicentral distance in kilometers (Vudler and Raica, 1980); or

$$
M_{d}=-3.38+2.74 \log (D)
$$

where $D$ is the coda duration in seconds (Viret, 1980). The first of these is satisfactory for larger events $(M \geq 2.8)$ but overestimates smaller events, for which the second formula is used.

\section{SEISMICITY IN THE SASZ}

A series of the cumulative seismicity maps in the southern Appalachian seismic zone from data collected by SARSN are shown in Figure 3a-3e and cumulative seismicity is listed in Appendix 1. The geological provinces in the southern Appalachian area, published by the U. S. Geological Survey, are indicated by the lines. The solid circles are the locations of the epicenters. The magnitude scale is represented by the size code as follows:

-4 to 4.9 ; -3 to 3.9 ; -2 to $2.9 ;-0.0$ to 2.0 ; $\times-$ less than 0.0 . They show that the level of activity concentrates more heavily in the Valley and Ridge province of eastern Tennessee, as opposed to the Blue Ridge or Inner Piedmont. The large majority of these events lie between the New York - Alabama lineament and the Clingman/Ocoee lineaments, magnetic anomalies produced by deep-seated basement structures. In other words, this regional seismic network has proved adequate to define the essential first-order seismological characteristics of the SASZ.

One of the most important contributions of SARSN to the monitoring of seismicity in the SASZ is to provide good spatial coverage for earthquake locations especially in the eastern Tennessee. A cluster of earthquake epicenters delineating a zone parallel to the geological provinces is evident in Figure 3a-3e.

\section{ACTIVE FAULTS IN EASTERN TENNESSEE}

As stated earlier, this network has a station density of 50-130 $\mathrm{km}$ in eastern Tennessee. All but 3 of the 18 permanent seismic stations have single component vertical sensors, and all but a few have modest dynamic range (40-60 dB). Figure 4(a) and 4(b) are the cumulative focal depth distribution of the southeastern U. S. earthquakes, including reservoir activity, for the digital data between October, 1986 and December, 1991, and analog data between September, 1981 and September, 1986. Both show that depths range down to 30 $\mathrm{km}$ but concentrate between 10 and $16 \mathrm{~km}$, occurring principally beneath the Appalachian overthrust. This pattern of depth distribution is similar to that reported by Johnston et al (1985) based on only two years of SARSN data.:

Figure $5 \mathrm{a}$ is the index map for the cross-sectional views of seismicity in Valley and Ridge province, eastern Tennessee. A number of cross-sectional views of SASZ seismicity 


\section{S. Appalachian Seismicity (dep $\geq 5$., mag $\geq 0$.): 1981 - 1992}

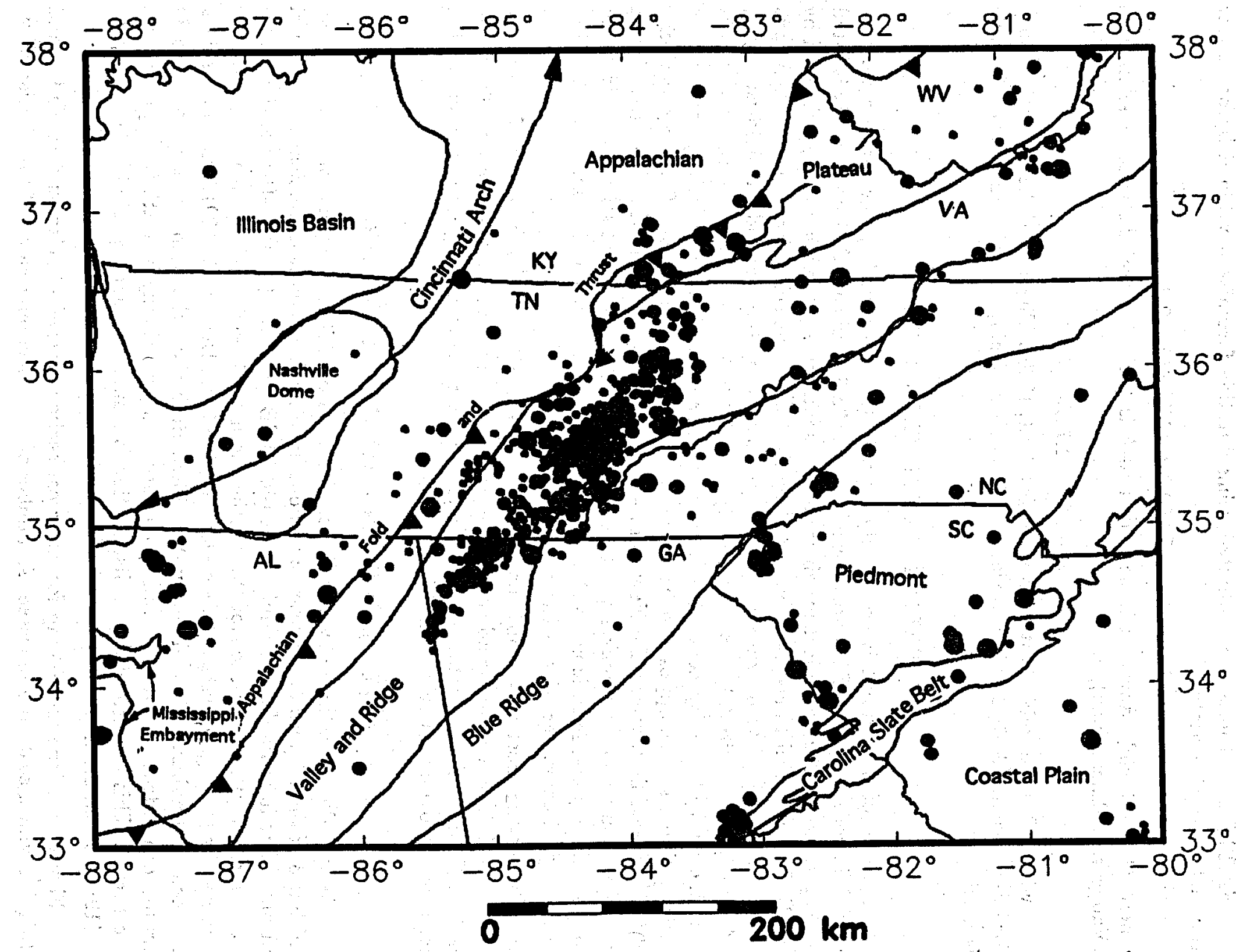

Figure 3a. Cumulative earthquake activity in the southern Appalachian with magnitude greater than or equal to 0.0 and focal depth greater than or equal to $5.0 \mathrm{~km}$ recorded by SARSN between 1981 and 1992. 


\section{S. Appalachian Seismicity ( mag $\geq 3$. ): 1981 - 1992}

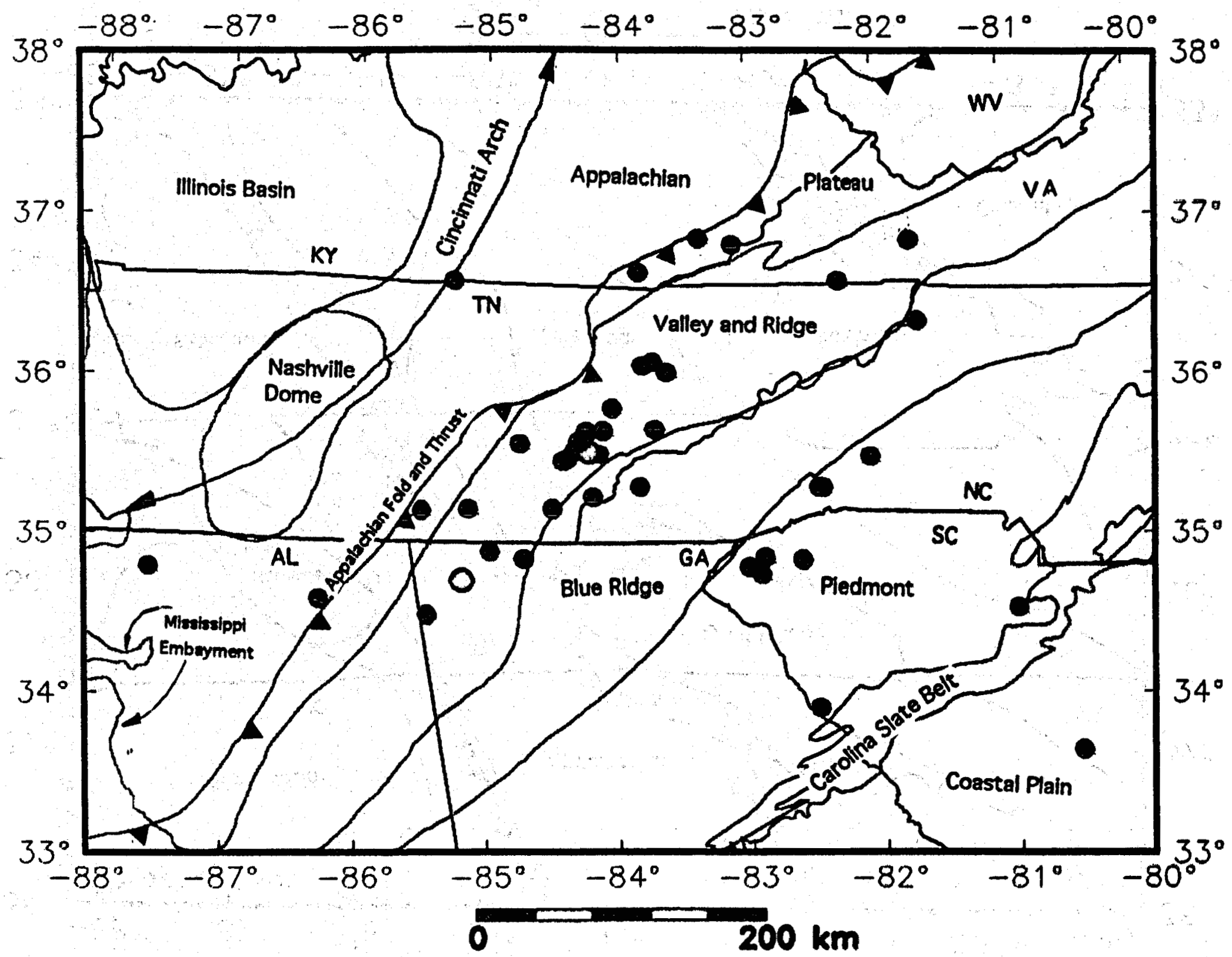

Figure 3b. Cumulative earthquake activity in the southern Appalachian with magnitude greater than or equal to 3.0 recorded by SARSN between 1981 and 1992. 


\section{S. Appalachian Seismicity ( q : A ): $1981-1992$}

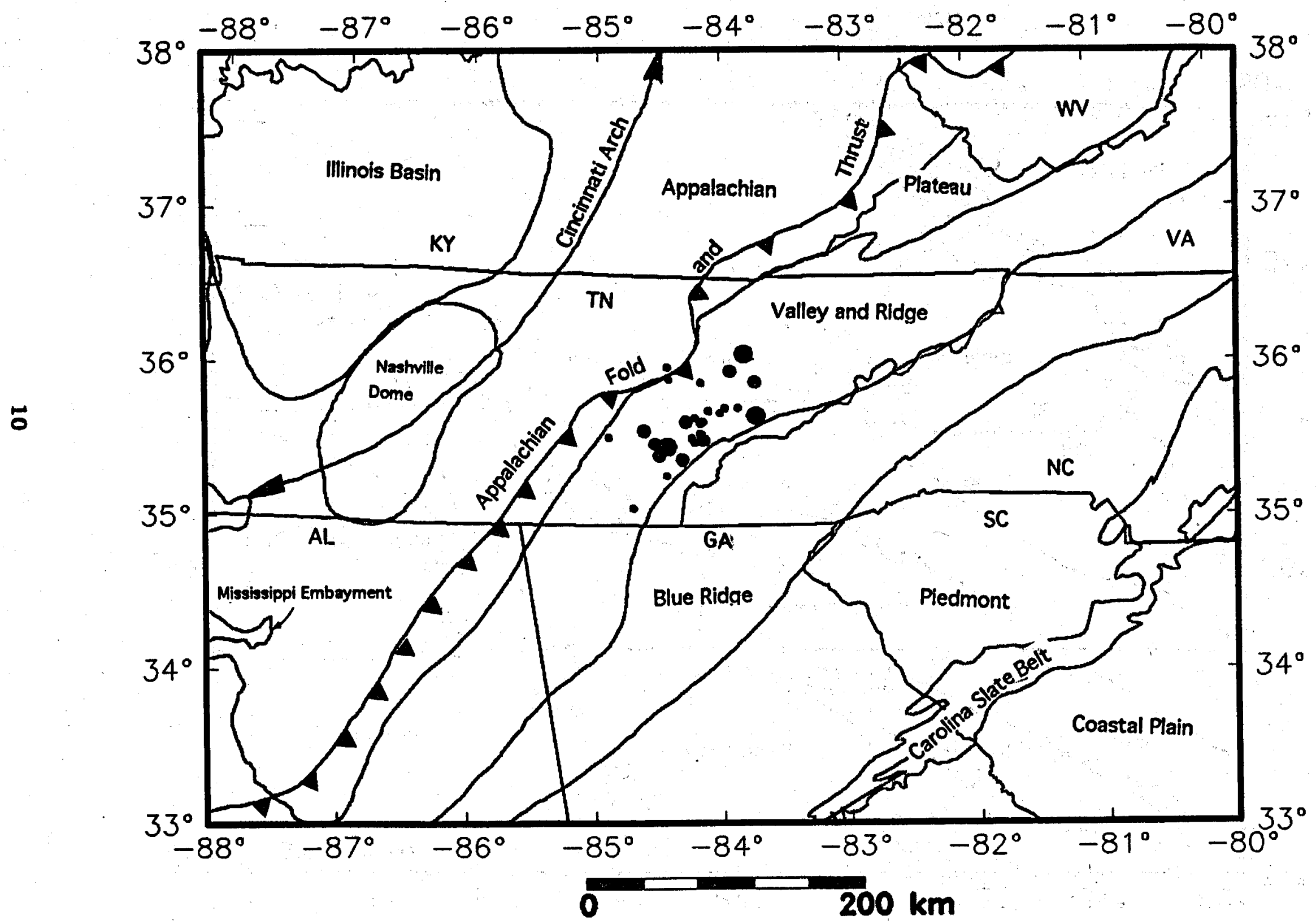

Figure 3c. Cumulative southern Appalachian seismicity recorded by SARSN between 1981 and 1992 with quality $A$ of the hypocentral location. 


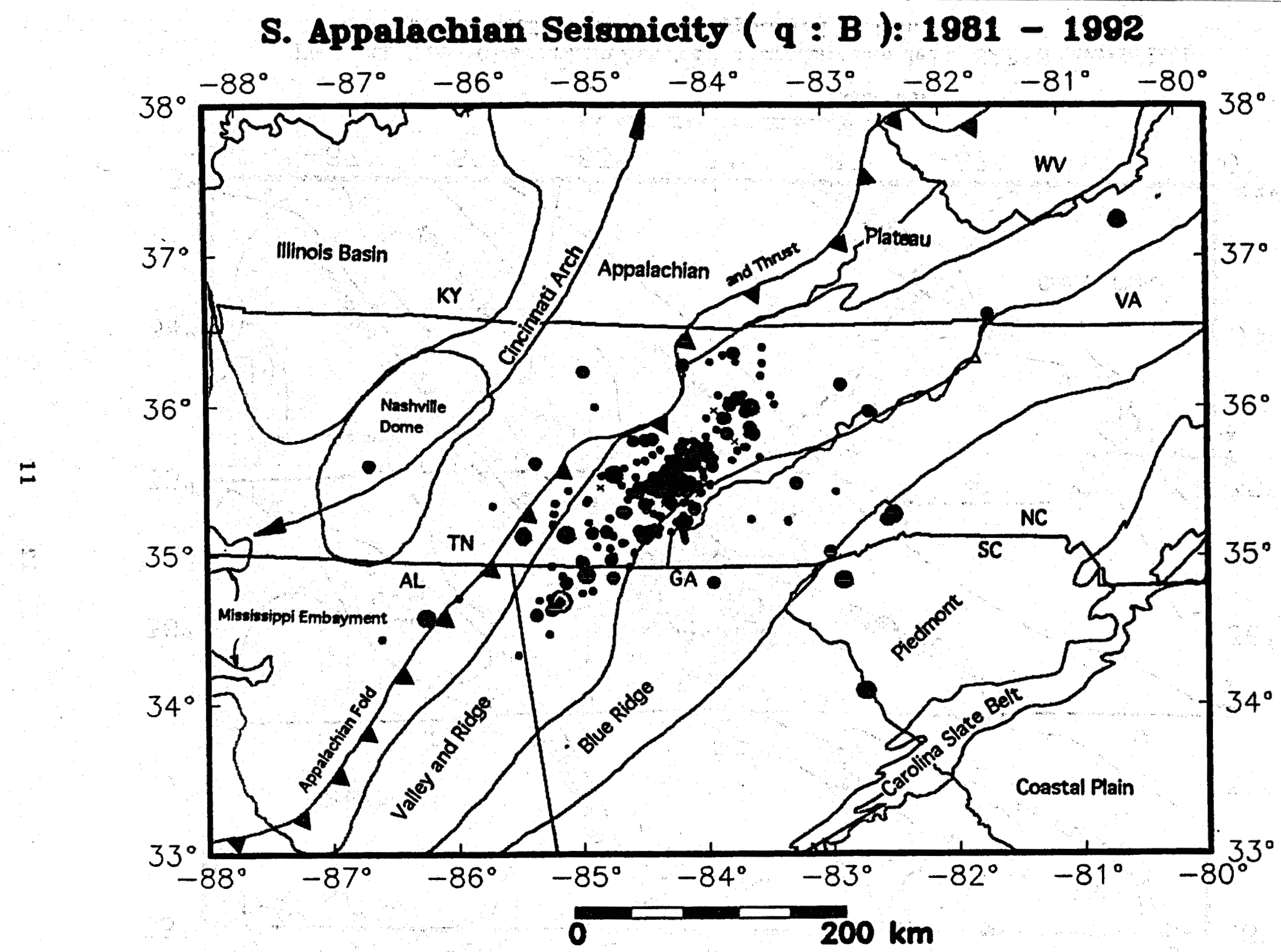

… Figure 3d. Cumulative southern Appalachian seismicity recorded by SARSN between 1981 and 1992 with quality $B$ of the hypocentral location . 


\section{S. Appalachian Seismicity ( $q$ : A\&B ): 1981 - 1992}

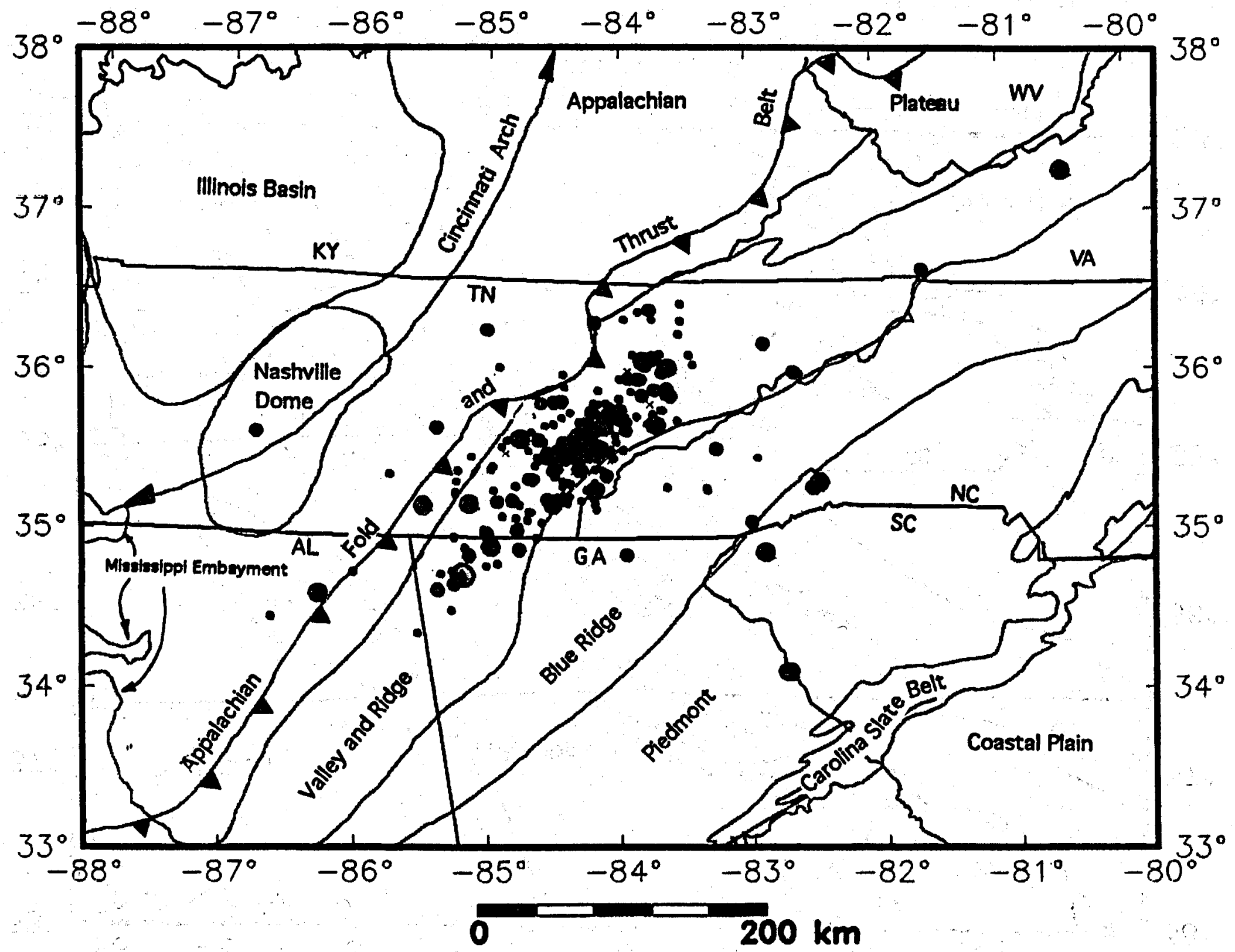

Figure 3e. Cumulative southern Appalachian seismicity recorded by SARSN between 1981 and 1992 with quality A or B of the hypocentral location. 


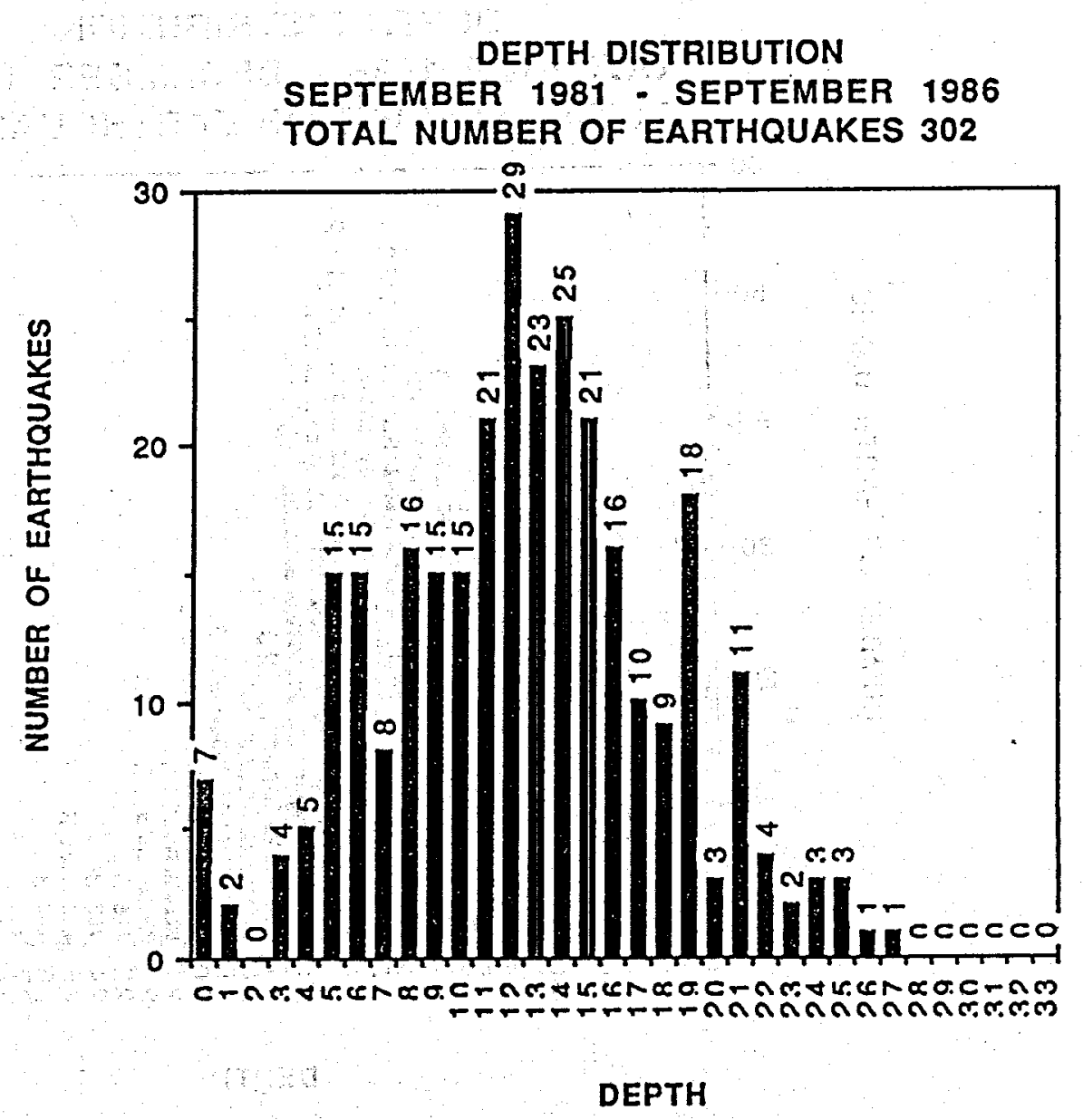

Figure 4a. Depth distribution of the CERI analog earthquake catalog between September, 1981 and September, 1986. Depths concentrate in a range of $8-16 \mathrm{~km}$. 


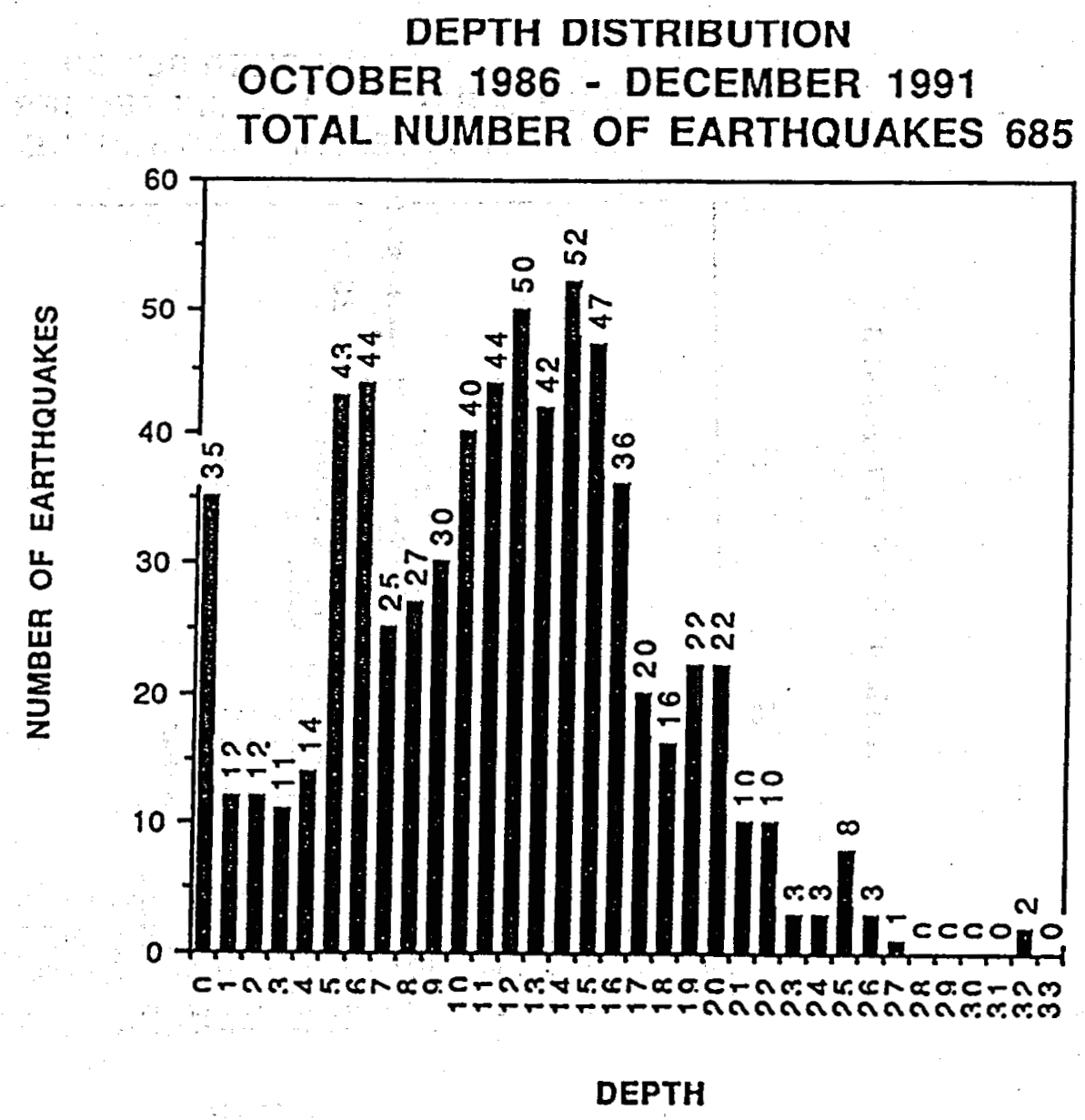

Figure 4b. Depth distribution of the CERI digital earthquake catalog between October, 1986 and December, 1991. Depths concentrate in a range of $10-16 \mathrm{~km}$. 
S. Appalachian Seismicity: 1981 - 1982

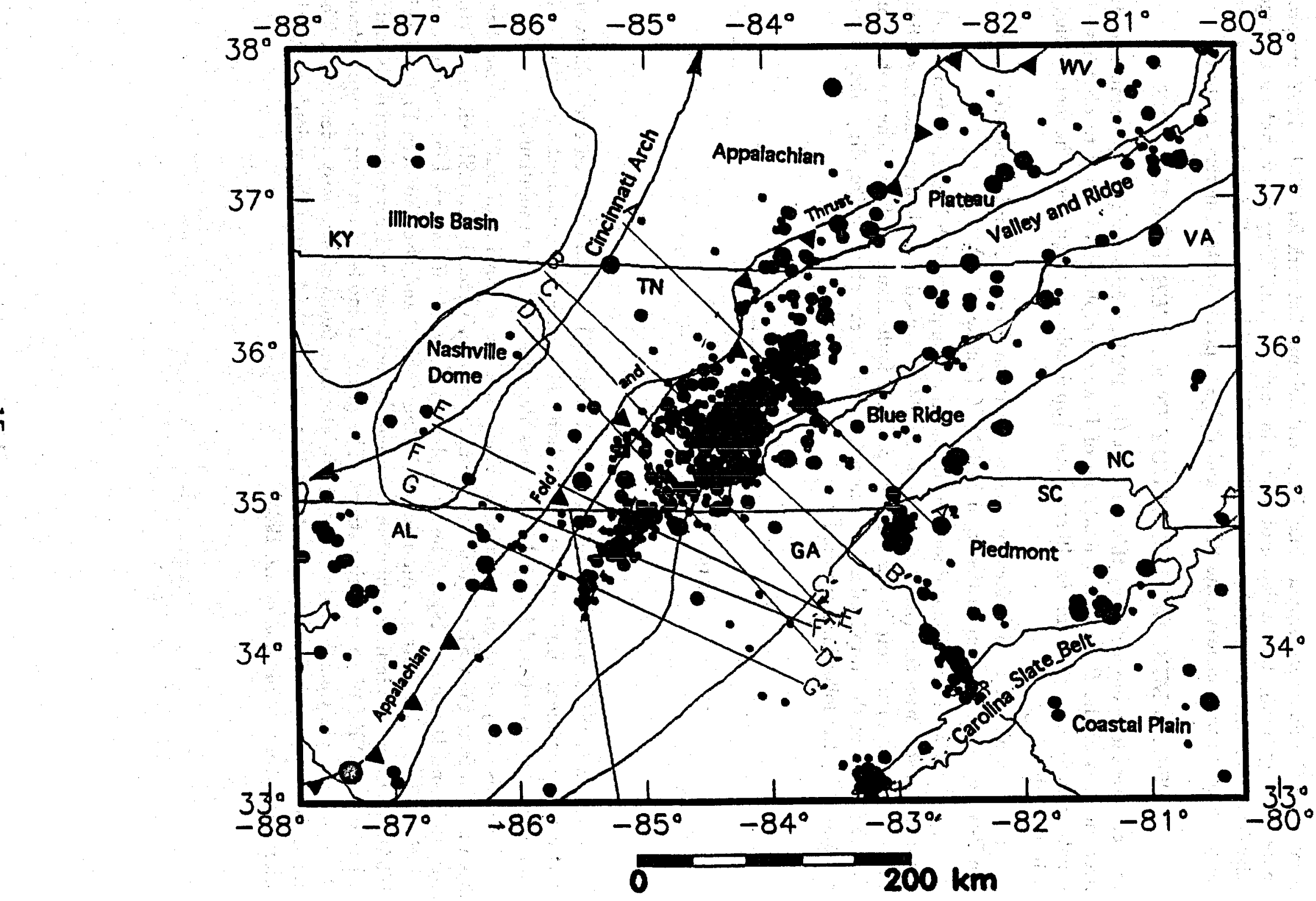

Figure 5a. Index Map for the cross-sectional views for the Figure $5 b$ in the Valley and Ridge province of the southern Appalachian seismic zone. 
located by the Southern Appalachian Regional Seismic Network for 1981 - 1992 are presented in Figure $5 \mathrm{~b}$. It is a depth of $60 \mathrm{~km}$ in each section. They show that on average seismicity is shallower to the east beneath the Blue Ridge and Piedmont provinces and deepens to the west beneath the Valley and Ridge and the North American craton.

\section{FOCAL MECHANISM STUDY IN EASTERN TENNESSEE}

In recent research supported by this contract $(\mathrm{Li}, 1994)$, a grid-searching procedure using first motions of the $\mathrm{P}$-wave and modeling of $\mathrm{P}$-wave amplitudes is used to obtain wellconstrained focal mechanisms for 19 southern Appalachian earthquakes. The methodology is in the following.

(1). A family of possible focal mechanism solutions of each event is generated by using the P-wave first-motion polarities from FOCMEC (Snoke, 1984).

(2). A forward modeling of focal mechanism technique, which is derived from Ebel's moment tensor inversion (Ebel and Bonjer, 1990), is used to calculate the theoretical vertical displacement amplitudes for each possible fault plane solution. The root-meansquare (RMS) of the amplitudes for each set of fault parameters (strike, dip, rake) is also calculated by the following equation,

$$
R M S=\sqrt{\frac{1}{n} \sum_{i=1}^{n}[O(i)-C(i)]^{2}}
$$

where $n$ is the number of amplitude; $O(i)$ and $C(i)$ are the observed and calculated vertical displacement amplitudes at one station. The preferred focal mechanism that has minimum RMS is selected from all these possible focal mechanism solutions.

For this study, nineteen events in the Valley and Ridge province of east Tennessee have been chosen from the CERI digital earthquake catalog collected by SARSN between October, 1986 and December, 1991. The fault plane parameters of preferred focal mechanism solutions for these events are summarized in the Table 2 and also shown in Figure 6 ( $\mathbf{L i}$, 1994). These results indicate that the basement of the Valley and Ridge province is under a horizontal, NE-SW compressive stress. The principal mode of faulting is right-lateral strike-slip on nearly north-south faults or left-lateral on faults of east-west orientation. The north-south orientation is favored because it agrees with the trend of the region's magnetic anomaly pattern (Johnston et al., 1985). 

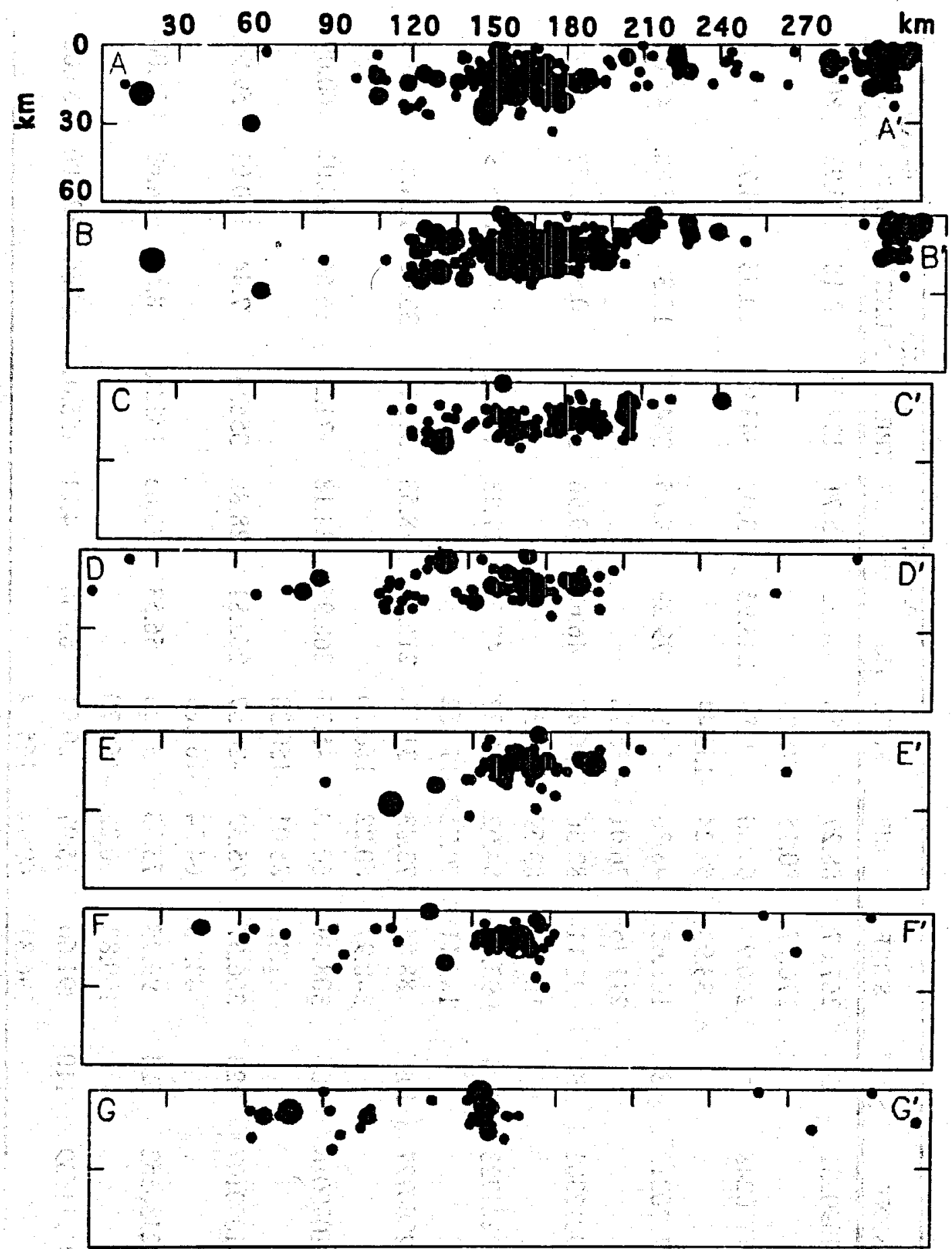

Figure 5b. 7 cross-sections of depth distribution indicated in Figure 5a. It is a depth of $60 \mathrm{~km}$ in each section. They show that on average seismicity is shallower to the east beneath the Blue Ridge and Piedmont provinces and deepens to the west beneath the Valley and Ridge province and the North American craton. 
Table 2. Summary of Focal Mechanisms

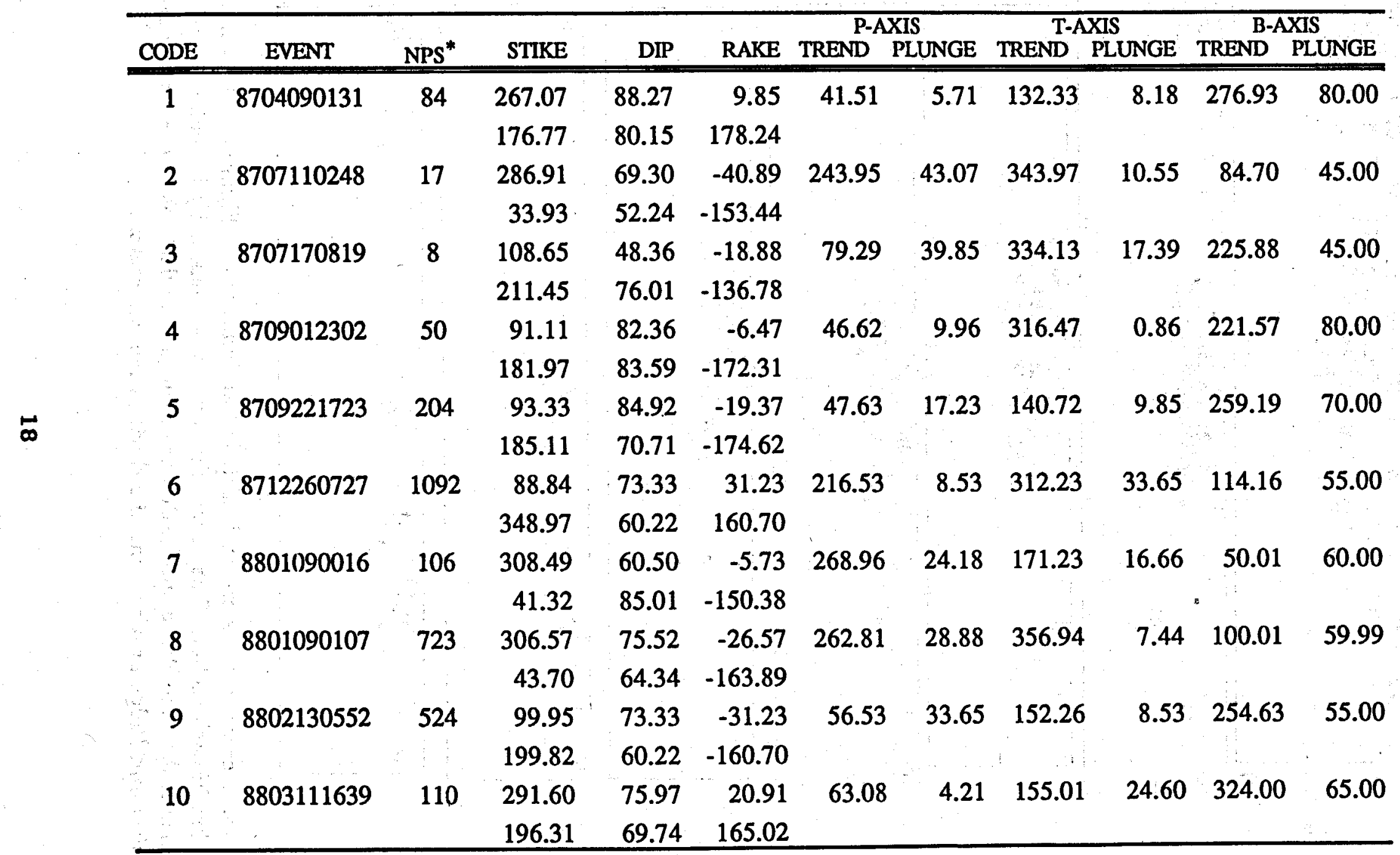


Table 2. (continued)

\begin{tabular}{|c|c|c|c|c|c|c|c|c|c|c|c|}
\hline \multirow{2}{*}{ CODE } & \multirow{2}{*}{ VENT } & \multirow{2}{*}{ NPS ${ }^{*}$} & \multirow{2}{*}{ STIKE } & \multirow{2}{*}{ DIP } & \multirow{2}{*}{ RAKE } & \multicolumn{2}{|c|}{$\overline{\text { P-AXIS }}$} & \multicolumn{2}{|c|}{ T-AXIS } & \multicolumn{2}{|c|}{$\overline{\text { B-AXIS }}$} \\
\hline & & & & & & & & & & & \\
\hline \multirow[t]{2}{*}{11} & 8804230101 & 594 & 100.78 & 76.00 & 14.43 & 54.00 & 0.01 & 324.00 & 20.00 & 144.00 & 70.00 \\
\hline & & & 7.22 & 76.01 & 165.56 & & & & & & \\
\hline \multirow[t]{2}{*}{12} & 8804240008 & 276 & 289.43 & 83.59 & -39.57 & 239.31 & 31.77 & 343.53 & 21.63 & 101.74 & 50.00 \\
\hline & & & 24.70 & 50.73 & -171.71 & & & & & & \\
\hline \multirow[t]{2}{*}{13} & 8807261927 & 294 & 256.23 & 75.06 & 1.34 & 212.03 & 9.57 & 120.07 & 11.44 & 341.05 & 75.00 \\
\hline & & & 165.88 & 88.71 & 165.06 & & & & & & \\
\hline \multirow[t]{2}{*}{14} & 8811011634 & 1260 & 284.33 & 87.50 & -29.91 & 235.90 & 22.53 & 333.99 & 18.75 & 99.99 & 60.00 \\
\hline & & & 15.77 & 60.12 & -177.12 & & & & & & \\
\hline \multirow[t]{2}{*}{15} & 8907111453 & 638 & 75.33 & 86.17 & 49.89 & 196.89 & 29.50 & 311.09 & 35.93 & 78.55 & 40.00 \\
\hline & & & 340.80 & 40.26 & 174.07 & & & & & & \\
\hline \multirow[t]{2}{*}{16} & 8909070518 & 897 & 255.59 & 71.11 & -47.21 & 209.38 & 46.05 & 315.74 & 15.19 & 58.91 & 40.00 \\
\hline & & & 4.87 & 46.03 & -153.26 & & & & & & $\therefore$ \\
\hline \multirow[t]{2}{*}{17} & 9007111841 & 1469 & 258.62 & 74.81 & -48.24 & 209.49 & 43.97 & 318.77 & 18.89 & 65.45 & 40.00 \\
\hline & & & 4.98 & 43.96 & -157.82 & & & & & & \\
\hline \multirow[t]{2}{*}{18} & 9105290247 & 1223 & 90.00 & 60.00 & 0.00 & 49.11 & 20.70 & 310.89 & 20.70 & 180.00 & 60.00 \\
\hline & $\cdots$ & & 180.00 & 90.00 & -150.00 & & & & & & \\
\hline \multirow[t]{2}{*}{19} & 9109240721 & 5 & 268.19 & 69.75 & -52.31 & 220.81 & 50.33 & 331.42 & 16.27 & 73.22 & 35.00 \\
\hline & & & 22.32 & 42.06 & -148.89 & & & & & & \\
\hline
\end{tabular}

* NPS - Number of Possible Solutions obtained by program FOCMEC ( $5^{\circ}$ increment searching). 


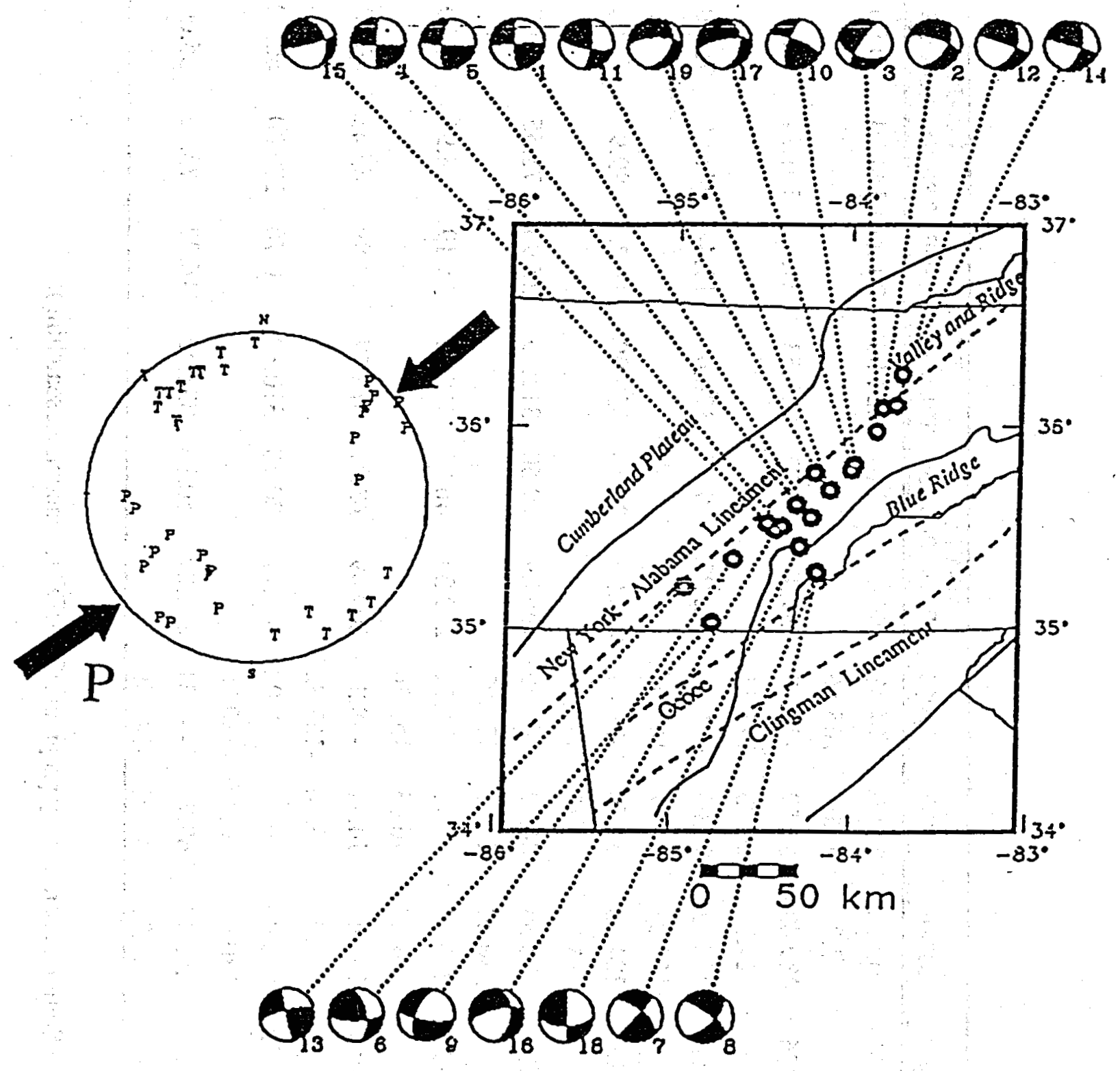

Figure 6. Locations of nineteen selected events in the Valley and Ridge province and their focal mechanisms. Black and white shaded areas in each focal sphere represent the compressional and dilatational quadrants respectively. $P$ and $T$ axes of nineteen focal mechanisms are also summarized in the left-handed focal sphere. The arrow indicates the average direction of the compressional stress in this region. 


\section{PUBLICATIONS}

CERI's publications based on SARSN data are as follows.

Bollinger, G. A., A. Teague, J. W. Munsey, and A. C. Johnston, (1985). Focal Mechanism Analyses for Virginia and Eastern Tennessee Earthquakes (1978-1984), NUREG/CR4288, U. S. Nuclear Regulatory Commission Washington, D.C., 83 pp.

Johnston, A.C., D.J. Reinbold, and S.I. Brewer, (1985). Seismotectonics of the Southern Appalachians, Bull. Seism. Soc. Am., 75, no. 1, pp. 291-312.

Johnston, A.C. and J.E. Zollweg, (1981). Two New Regional Seismic Networks in the Central and Southeastern United States, Earthquake Notes, 52, no. 2, pp. 66-71.

Li, H.S., (1994). A Focal Mechanism Analysis of Eastern Tennessee Seismic Zone Earthquakes, Thesis of Master of Science Degree, Memphis State University.

Long, L.T., K.-H. Zelt, J.-S. Liow, R. Propés, J. Shand, D. Reinbold, and B. Schechter, (1986). The North Georgia Earthquake of October 9, 1984, Earthquake Notes, 57, no. 3, pp. 77-82.

Nava, S.J., J.W. Munsey, and A.C. Johnston, (1989). First Fault Plane Identification in the Southern Appalachians: The mbLg 4.2. Vonore, Tennessee, Earthquake of March 27, 1987, Seismol. Res. Lett., 60, no. 3, pp. 119-129.

Powell, C.A., G.A. Bollinger, M.C. Chapman, A.C. Johnston, and R.L. Wheeler (1994). A Seismotectonic Model for Eastern Tennessee, in press, Science.

Reinbold, D.J. and A.C. Johnston, (1987). Historical Seismicity of the Southern Appalachian Seismic Zone, U. S. Geological Survey Open-File Report 87-433, 674 pp.

Sibol, M.S., J.A. Snoke, G.A. Bollinger, M.C. Chapman, J.B. Birch, and A.C. Johnston, (1990). The Probability of a Major Earthquake in the Eastern United States, Seismol. Res. Lett., 61, no. 2, pp. 131-134.

Teague, A.G., G.A. Bollinger, and A.C. Johnston, (1986). Focal Mechanism Analysis for Eastern Tennessee Earthquakes (1981-1989), Bull. Seism. Soc. Am., 76, no. 1, pp. 95-109.

Yao, P. and J. Dorman, (1992). Short-Period Surface-Wave Dispersion and Shallow Crustal Structure of Central and Eastern Tennessee, Bull. Seism. Soc. Am., 82, no. 2 , pp. 962-979. 


\section{REFERENCES}

Bollinger, G.A., M.C. Chapman, and T.P. Moore (1980), Central Virginia Regional Seismic Network: Crustal Velocity Structure in Central and Southwestern Virginia, NUREG/CR1217.

Bollinger, G.A., M.S. Sibol, and E.C. Mathena (1984), Seismicity of the Southeastern United States, Southeastern U.S. Seismic Network Bulletin, No. 19.

Ebel, J.E. and K.P. Bonjer (1990), Moment Tensor Inversion of Small Earthquakes in Southwestern Germany for The Fault Plane Solution, Geophys. J. Int., 101, pp.139146 .

Johnston, A.C., D. Reinbold, and S. Brewer (1985), Seismotectonics of the Southern Appalachians, Bulletin of the Seismological Society of America], vol. 75, No. 1, pp. 291-312.

Lahr, J.C. (1980), HYPOELLIPSE/MULTICS: A Computer Program for Determining Local Earthquake Hypocentral Parameters, Magnitude and First-Motion Pattern, USGS Open File Report 80-59.

Lee, W.H.K., and J. Lahr, (1975), HYPO71 (revised): A Computer Program for Determining Hypocenter, Magnitude, and First Motion Pattern of Local Earthquakes, USGS Open File Report 75-911.

Snoke, J.A., J.W. Munsey, A.G. Teague, and G.A. Bollinger (1984). A Program for Focal Mechanism Determination by Combined Used of Polarity and SV.P Amplitude ratio data, Earthquake Notes 55, pp. 15.

Stauder, W., R. Herrmann, S. Singh, R. Perry, E. Haug, and S.T. Morrisey (1980), Central Mississippi Valley Earthquake Bulletin, Quarterly Report No. 23, Saint Louis University.

Viret, M., (1980), Determination of a duration magnitude relationship for the Virginia Tech Seismic Network, in Bollinger, G.A., Central Virginia Regional Seismic Report Dec. 1, 1980, U.S. Nuclear Regulatory Commission Contract No. NRC-04-077-094, p.1-8.

Vudler, V., and P. Raica (1980), Seismicity of the Northeastern United States, Bulletin No. 19, Weston Observatory, Boston College. 
Appendix 1.

SOUTHERN APPALACHIAN (SARSN) HYPOCENTERS; CUMULATIVE LISTING SEPTEMBER 1081 - DECEMBER 1002

(A). STUDY AREA EARTHQUAKES (SARSN IS PRIMARY NETWORK)

All events from September 1981 - December 1985 were located using HYPO71.

DATE ORGIN LAT $^{\circ} \mathrm{N}$ LONG $^{\circ} \mathrm{W}$ DEP MAG ST|PH DMIN GAP Q SQD RMS ERH1 ERH2 ERZ AZM

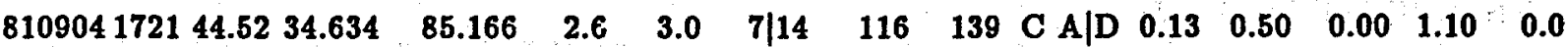
GA, CATLETT QUAD

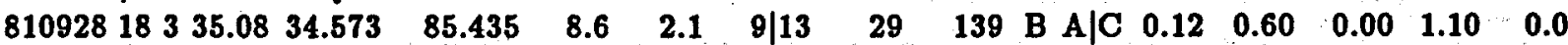
GA, DOUGHERTY QUAD

$\begin{array}{lllllllllllllllll}811125 & 1154 & 26.10 & 35.647 & 84.642 & 12.7 & 2.9 & 13 / 23 & 35 & 74 & \text { B A A C } & 0.13 & 0.40 & 0.00 & 0.60 & 0.0\end{array}$

TN, TEN MILE QUAD

$\begin{array}{llllllllllllllll}811204 & 446 & 20.40 & 35.295 & 84.004 & 10.7 & 1.9 & 8 \mid 11 & 41 & 189 & \text { C B|D } & 0.24 & 1.70 & 0.00 & 4.60 & 0.0\end{array}$

TN, BIG JUNCTION QUAD; TN/NC BORDER

$\begin{array}{llllllllllllllllll}811213 & 942 & 34.00 & 34.985 & 85.143 & 4.0 & 0.6 & 3 \mid 5 & 19 & 152 & \text { C B } & 0.04 & 0.04 & 1.00 & 0.00 & 3.20 & 0.0\end{array}$

GA, EAST RIDGE QUAD; GA/TN BORDER

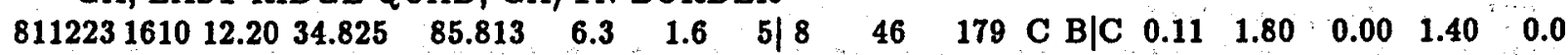

AL, STEVENSON QUAD

$\begin{array}{lllllllllllllllll}811225 & 163 & 31.43 & 35.470 & 84.178 & 10.2 & 2.3 & 7 / 13 & 30 & 136 & \text { B A } \mid \mathrm{C} & 0.12 & 0.60 & 0.00 & 0.80 & 0.0\end{array}$

TN, RAFTER QUAD

$\begin{array}{llllllllllllllllllllllll}820102 & 2 & 0 & 26.20 & 35.179 & 86.429 & 12.9 & 3.1 & 9 \mid 13 & 63 & 138 & \text { C B B } & 0.17 & 0.70 & 0.00 & 2.60 & 0.0\end{array}$ TN, MULBERRY QUAD; FELT LINCOLN, FRANKLIN, MOORE \& MARSHALL COUNTIES, TN

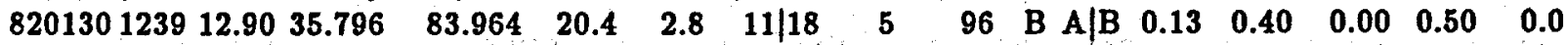
TN, MARYVILLE QUAD

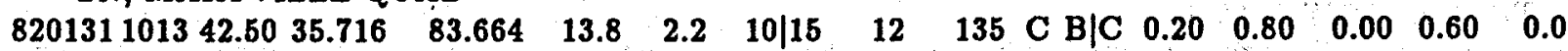
TN, WEAR COVE QUAD

$\begin{array}{lllllllllllllllll}820223 & 919 & 8.80 & 34.575 & 85.445 & 6.5 & 2.3 & 11 \mid 17 & 51 & 128 & \text { C B|D } & 0.20 & 0.70 & 0.00 & 1.50 & 0.0\end{array}$ GA, DOUGHERTY QUAD

$\begin{array}{llllllllllllllllll}820224 & 1210 & 6.30 & 35.733 & 84.304 & 20.5 & 1.5 & 4 & 7 & 20 & 163 & \text { B A A C } & 0.04 & 0.30 & 0.00 & 1.00 & 0.0\end{array}$ TN, LENOIR CITY QUAD

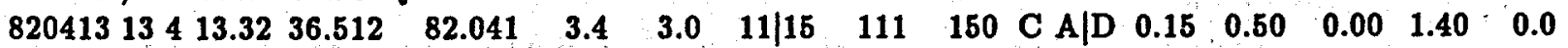
TN, HOLSTON VALEY QUAD; TN/VA BORDER

$\begin{array}{llllllllllllllllll}820505 & 1528 & 16.60 & 35.675 & 84.415 & 13.0 & 1.9 & 6 \mid 9 & 39 & 243 & \mathrm{C} & \mathrm{B} \mid \mathrm{D} & 0.10 & 1.20 & 0.00 & 1.30 & 0.0\end{array}$ TN, PHILADELPHIA QUAD

$\begin{array}{lllllllllllllllllllll}820512 & 121 & 52.20 & 34.896 & 85.020 & 10.0 & 2.6 & 13 \mid 25 & 31 & 89 & \text { B A } & \text { AC } & 0.14 & 0.30 & 0.00 & 0.60 & 0.0\end{array}$ GA, RINGGOLD QUAD; GA/TN BORDER

$\begin{array}{llllllllllllllllllllllll}820515 & 354 & 47.40 & 35.679 & 84.345 & 21.2 & 1.6 & 5 & 9 & 26 & 143 & \text { B A A C } & 0.14 & 0.90 & 0.00 & 1.70 & 0.0\end{array}$ TN, LOUDON QUAD

$\begin{array}{lllllllllllllllllllllll}820520 & 712 & 7.70 & 35.039 & 85.148 & 10.7 & 1.3 & 8 & 13 & 20 & 161 & \text { B A } & \text { A C } & 0.12 & 0.60 & 0.00 & 0.60 & 0.0\end{array}$ $\begin{array}{llllllllllllllllllll}\text { TN, CHATTANOOGA QUAD } & & & & & \end{array}$ GA, FORT OGLETHORPE QUAD; GA/TN BORDER

$\begin{array}{lllllllllllllllllll}820530 & 711 & 59.10 & 35.702 & 84.135 & 11.6 & 1.4 & 10 \mid 14 & 19 & 81 & \text { B A A|B } & 0.09 & 0.30 & 0.00 & 0.60 & 0.0\end{array}$ TN, MEADOW QUAD

$\begin{array}{llllllllllllllllllllll}820612 & 1220 & 29.50 & 36.093 & 83.777 & 16.3 & 1.1 & 3 & 5 & 21 & 333 & \text { C A } & 0.03 & 0.60 & 0.00 & 0.70 & 0.0\end{array}$ TN, JOHN SEVIER QUAD

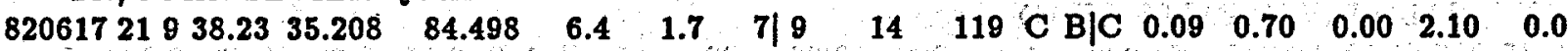
TN, MC FARLAND QUAD

$\begin{array}{lllllllllllllllllllllllllll}820708 & 518 & 50.90 & 35.484 & 84.178 & 15.2 & 0.7 & 5 & 8 & 23 & 137 & \text { B A } & 0.08 & 0.60 & 0.00 & 1.40 & 0.0\end{array}$ TN, RAFTER QUAD 
DATE ORGIN LAT ${ }^{\circ} \mathrm{N}$ LONG ${ }^{\circ} \mathrm{W}$ DEP MAG ST|PH DMIN GAP $Q$ SQD RMS ERH1 ERH2 ERZ AZM

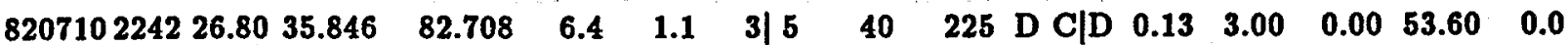
NC, MARSHALL QUAD

$\begin{array}{lllllllllllllllll}820719 & 434 & 42.60 & 35.738 & 83.978 & 12.6 & 0.9 & 4 \mid 6 & 21 & 212 & \text { C A } & \text { A } & 0.04 & 0.40 & 0.00 & 0.70 & 0.0\end{array}$ TN, BLOCKHOUSE QUAD

$\begin{array}{llllllllllllllllll}820825 & 744 & 47.78 & 35.922 & 83.906 & 14.3 & 1.5 & 7 \mid 14 & 2 & 172 & \text { B A } A \mid C & 0.12 & 0.60 & 0.00 & 0.40 & 0.0\end{array}$ TN, KNOXVILLE QUAD

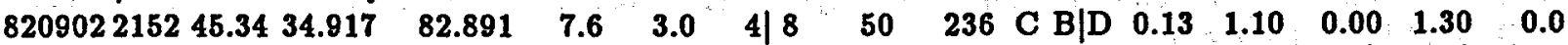
SC, SALEM QUAD

$\begin{array}{llllllllllllllllll}820905 & 1011 & 9.40 & 35.192 & 84.506 & 12.6 & 3.2 & 11 \mid 16 & 16 & 101 & \text { B } & \text { A } \mid B & 0.09 & 0.30 & 0.00 & 0.30 & 0.0\end{array}$ TN, OSWALD DOME QUAD; FELT AROUND ETOWAH, TN

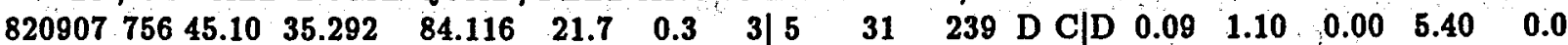

TN, BIG JUNCTION QUAD TN/NC BORDER

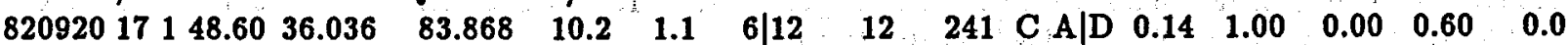
TN, JOHN SEVIER QUAD

$\begin{array}{llllllllllllllll}820921 & 17 & 18.30 & 36.113 & 83.750 & 15.7 & 1.4 & 10 \mid 17 & 24 & 226 & \text { C A|D } & 0.11 & 0.60 & 0.00 & 0.90 & 0.0\end{array}$ TN, GRAVESTON QUAD

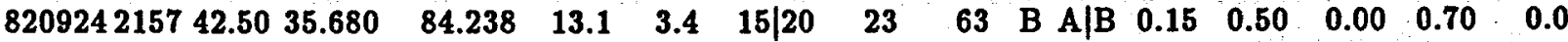
TN, MEADOW QUAD; FELT IN KNOX, LOUDON, BLOUNT, ANDERSON \& MONROE COUNTIES

\begin{tabular}{llllllllll|llllll}
8209242219 & 16.95 & 35.684 & 84.251 & 8.1 & 3.5 & $16 \mid 22$ & 43 & 62 & B A|C & 0.14 & 0.40 & 0.00 & 0.70 & 0.0
\end{tabular} TN, LOUDON QUAD; FELT IN KNOX, LOUDON, BLOUNT, ANDERSON MONROE \& MCMINN COUNTIES

$820924225411.8835 .681 \quad 84.241$

TN, MEADOW QUAD

$\begin{array}{llllllllllllllllllllll}820926 & 234 & 1.52 & 35.678 & 84.271 & 4.4 & 0.0 & 3 / 4 & 1 & 234 & \text { C A|D } & 0.08 & 0.00 & 0.00 & 0.00 & 0.0\end{array}$

TN, LOUDON QUAD

$\begin{array}{lllllllllllllllll}820927 & 748 & 25.97 & 35.683 & 84.239 & 14.5^{\circ} & 0.0 & 6 / 7 & 4 & 94 & \text { B } & \text { B|B } & 0.09 & 1.30 & 0.00 & 0.70 & 0.0\end{array}$

TN, MEADOW QUAD

$821018 \quad 426 \quad 27.04 \quad 35.683 \quad 84.239$

TN, MEADOW QUAD

$821022 \quad 23432.50 \quad 36.324 \quad 84.067$

TN, DEMORY QUAD

$\begin{array}{lllllllllllllllllllllll}821102 & 844 & 45.64 & 35.766 & 83.970 & 13.6 & 1.2 & 5 & \theta & 20 & 147 & \text { B A A C } & 0.09 & 0.50 & 0.00 & 0.70 & 0.0\end{array}$

TN, MARYVILLE QUAD

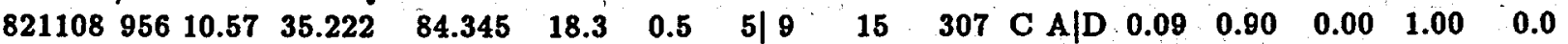

TN, FARNER QUAD; TN/NC BORDER

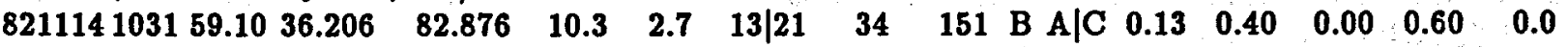

TN, MOSHEIM QUAD

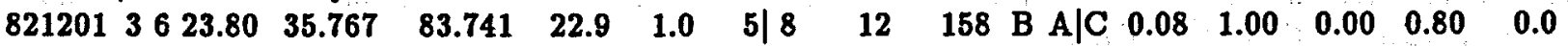
TN, WILDWOOD QUAD

$\begin{array}{lllllllllllllllll}821201 & 1339 & 44.60 & 35.255 & 84.428 & 13.1 & 0.4 & 6 \mid 12 & 8 & 109 & \text { B } & \text { A } \mid \text { B } & 0.12 & 0.50 & 0.00 & 0.80 & 0.0\end{array}$ TN, MECCA QUAD

$\begin{array}{llllllllllllllll}821206 & 1052 & 35.10 & 36.136 & 83.747 & 9.0 & 1.7 & 7 \mid 12 & 26 & 199 & \text { C A|D } & 0.12 & 0.80 & 0.00 & 0.80 & 0.0\end{array}$ TN, LUTTRELL QUAD

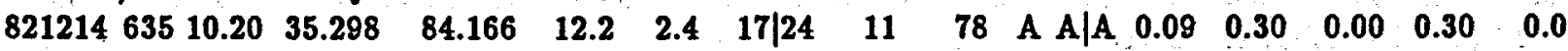
TN, BALD RIVER FALLS QUAD .

$\begin{array}{llllllllllllllllllllll}821215 & 227 & 59.30 & 35.749 & 84.220 & 13.1 & 2.1 & 13 \mid 22 & 20 & 81 & \text { B A|B } & 0.11 & 0.30 & 0.00 & 0.30 & 0.0\end{array}$ TN, CONCORD QUAD

$\begin{array}{llllllllllllllllll}830118 & 5 & 9 & 12.30 & 35.589 & 84.287 & 8.2 & 2.3 & 11 \mid 18 & 32 & 88 & \text { B A } & \text { A C } & 0.15 & 0.40 & 0.00 & 1.00 & 0.0\end{array}$ TN, MADISONVILLE QUAD 
DATE ORGIN LAT ${ }^{\circ} \mathrm{N}$ LONG ${ }^{\circ} W$ DEP MAG ST|PH DMIN GAP Q SQD RMS ERH1 ERH2 ERZ AZM

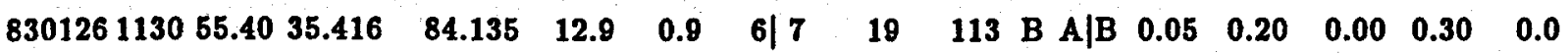

TN, RAFTER QUAD

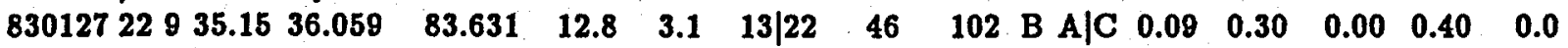

TN, MASCOT QUAD

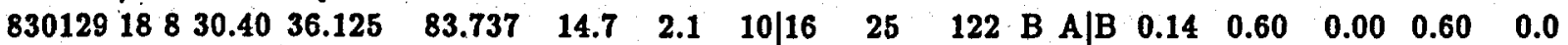

TN, LUTTRELL QUAD

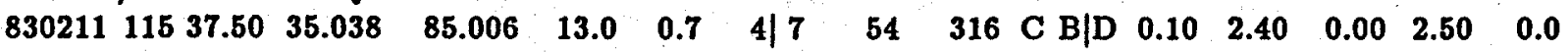

TN, OOLTEWAH QUAD

$83030414327.7035 .599 \quad 84.354$

TN, MADISONVILLE QUAD

$830311222939.93 \quad 35.210 \quad 84.422$

TN, MC FARLAND QUAD

$830313 \quad 353 \quad 12.61 \quad 35.507 \quad 84.352$

TN, MADISONVILLE QUAD

$830313142144.3035 .801 \quad 84.013$

TN, LOUISVILLE QUAD

$\begin{array}{llll}830316 & 913 & 52.13 \quad 35.228 & 84.551\end{array}$

TN, OSWALD DOME QUAD

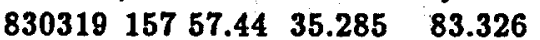

NC, GREENS CREEK QUAD

$\begin{array}{llllllllllll}12.5 & 2.3 & 7 / 12 & 9 & 145 & \text { B A } & \text { A C } & 0.10 & 0.40 & 0.00 & 0.50 & 0.0\end{array}$

$\begin{array}{llllllllllllll}16.7 & 1.7 & 5 & 8 & 13 & 168 & \text { B } & \text { A } \mid C & 0.02 & 0.20 & 0.00 & 0.30 & 0.0\end{array}$

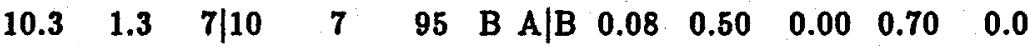

$\begin{array}{llllllllllllll}11.7 & 1.1 & 5 & 8 & 18 & 107 & \text { B A|B } & 0.11 & 0.70 & 0.00 & 1.20 & 0.0\end{array}$

$\begin{array}{llllllllllll}11.7 & 2.6 & 14 \mid 19 & 14 & 92 & \text { B } & \text { A|B } & 0.14 & 0.50 & 0.00 & 0.50 & 0.0\end{array}$

$\begin{array}{llllllllllll}2.5 & 1.6 & 8 \mid 14 & 32 & 150 & \text { B A } & \text { C } & 0.12 & 0.50 & 0.00 & 1.10 & 0.0\end{array}$

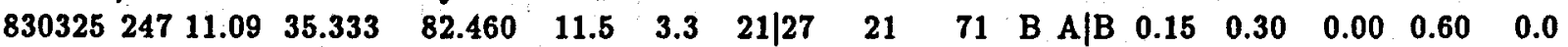

NC, HENDERSONVILLE QUAD; FELT IN HENDERSONVILLE, NC

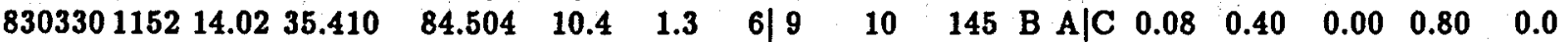

TN, ATHENS QUAD

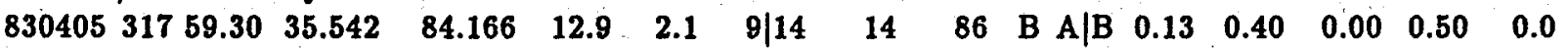

TN, VONORE QUAD

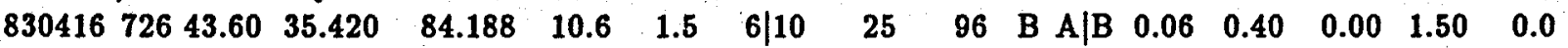

TN, RAFTER QUAD

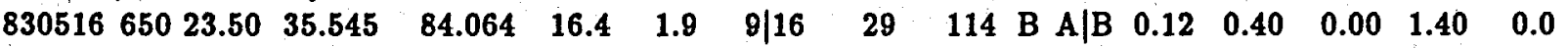
TN, TALLASSEE QUAD

$\begin{array}{llllllllllllllll}830525 & 1046 & 5.98 & 35.741 & 84.442 & 17.8 & 1.6 & 7 \mid 12 & 22 & 109 & \text { B A|B } & 0.13 & 0.50 & 0.00 & 1.50 & 0.0\end{array}$

TN, PHILADELPHIA QUAD

$83052612302.20 \quad 35.666 \quad 84.264$

TN, LOUDON QUAD

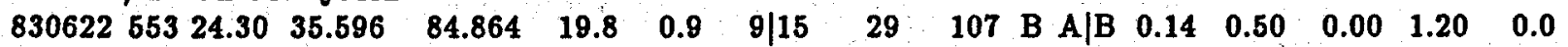

TN, DECATUR QUAD

$\begin{array}{lllllllllllllllllll}830626 & 1734 & 2.25 & 35.288 & 84.251 & 13.9 & 0.5 & 5 & 9 & 3 & 164 & \text { B A } & \text { AC } & 0.09 & 0.60 & 0.00 & 0.50 & 0.0\end{array}$ TN, TELLICO PLAINS QUAD

$\begin{array}{llllllllllllllll}830702 & 646 & 28.10 & 35.696 & 84.195 & 13.1 & 1.8 & 7 \mid 13 & 4 & 181 & \text { C A } \mid D & 0.11 & 0.60 & 0.00 & 0.40 & 0.0\end{array}$ TN, MEADOW QUAD

$\begin{array}{lllllllllllllllll}830708 & 1929 & 5.80 & 35.548 & 84.153 & 9.7 & 3.4 & 15 \mid 23 & 14 & 87 & \text { B A } \mid \text { B } & 0.10 & 0.20 & 0.00 & 0.50 & 0.0\end{array}$ TN, VONORE QUAD; FELT IN VONORE, TN

$\begin{array}{lllllllllllllllllllllll}830709 & 328 & 46.80 & 35.549 & 84.143 & 9.5 & 1.0 & 7 \mid 9 & 2 & 88 & \text { A A } \mid \mathrm{A} & 0.09 & 0.40 & 0.00 & 0.90 & 0.0\end{array}$

TN, VONORE QUAD

$\begin{array}{lllllllllllllllllllllllllll}830709 & 957 & 47.70 & 35.537 & 84.135 & 5.3 & 1.3 & 5 & 8 & 8 & 164 & \mathrm{C} & \mathrm{B} & \mathbf{C} & 0.07 & 1.20 & 0.00 & 2.00 & 0.0\end{array}$

TN, VONORE QUAD

$\begin{array}{lllllllllllllllll}830711 & 1254 & 36.09 & 35.636 & 86.789 & 29.3 & 2.4 & 9 \mid 14 & 23 & 98 & \text { B A } & \text { A } & 0.14 & 0.50 & 0.00 & 1.30 & 0.0\end{array}$ TN, RALLY HILL QUAD 
DATE ORGIN LAT $^{\circ} \mathrm{N}$ LONG ${ }^{\circ} \mathrm{W}$ DEP MAG ST|PH DMIN GAP $Q$ SQD RMS ERH 1 ERH2 ERZ AZM

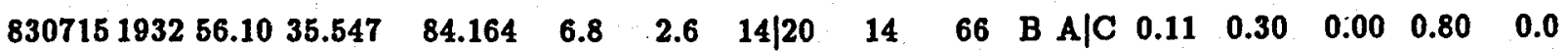
TN, VONORE QUAD

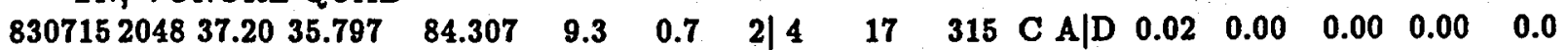
TN, LENOIR CITY QUAD

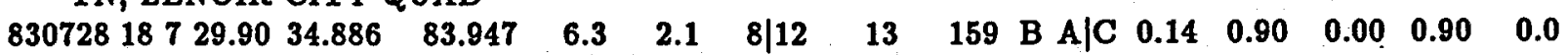
GA, BLAIRSVILLE QUAD; GA/NC BORDER

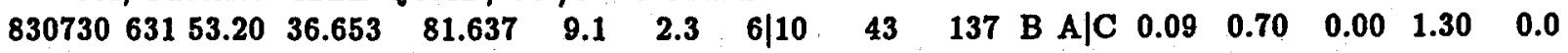
VA, KONNAROCK QUAD

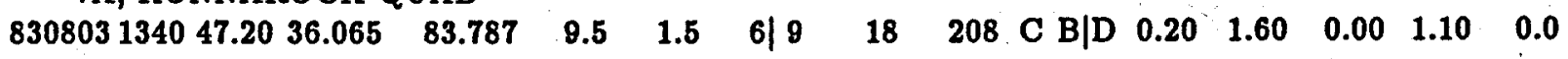
TN, JOHN SEVIER QUAD

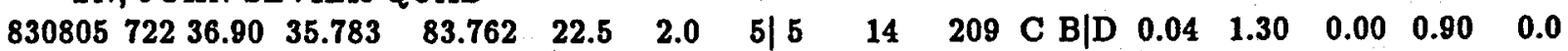
TN, WILDWOOD QUAD

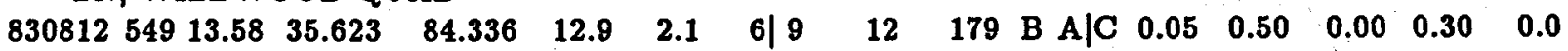

TN, LOUDON QUAD

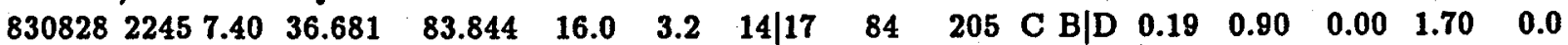
KY, KAYJAY QUAD; FELT IN MIDDLESBORO, KY AREA

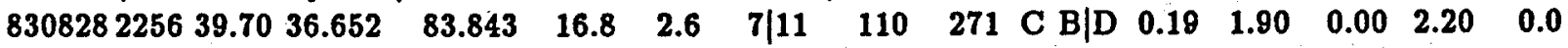
KY, KAYJAY QUAD

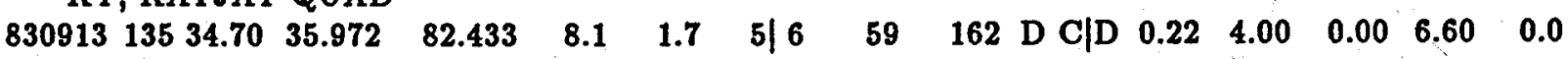
NC, BALD CREEK QUAD; NC/TN BORDER

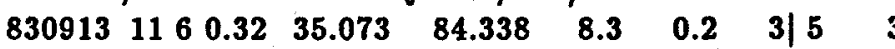
TN, ISABELLA QUAD; TN/NC BORDER

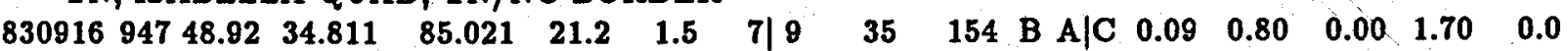
GA, TUNNEL HILL QUAD

$\begin{array}{lllllllllllllllll}831003 & 251 & 34.80 & 34.810 & 85.056 & 6.2 & 1.2 & 3 \mid 5 & 32 & 218 & \text { C B|D } & 0.05 & 1.50 & 0.00 & 2.70 & 0.0\end{array}$ GA, TUNNEL HILL QUAD

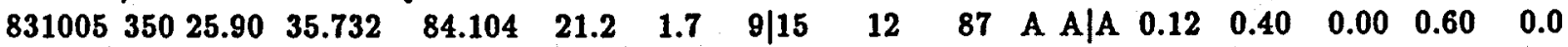
TN, BINFIELD QUAD

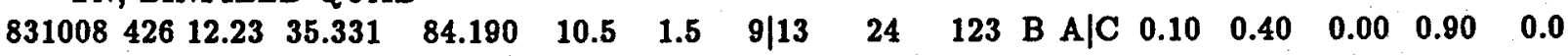
TN, BALD RIVER FALLS QUAD

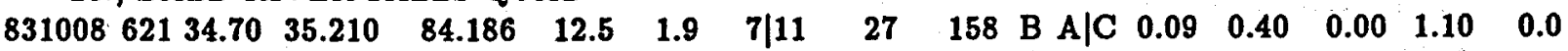
NC, UNAKA QUAD; NC/TN BORDER

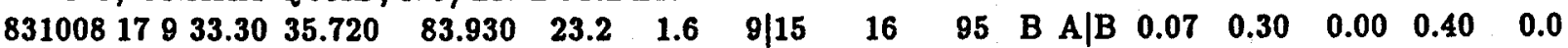
TN, BLOCKHOUSE QUAD

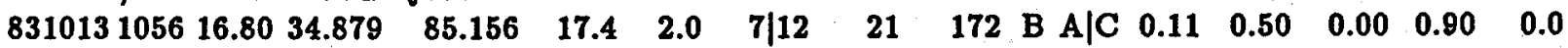
GA, EAST RIDGE QUAD; GA/TN BORDER

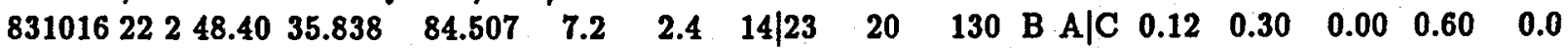
TN, BACON GAP QUAD

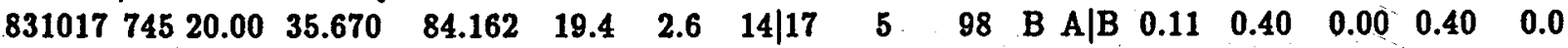
TN, MEADOW QUAD

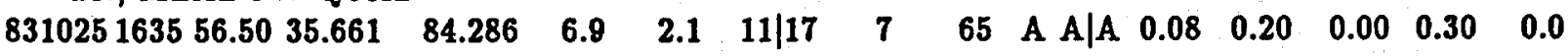

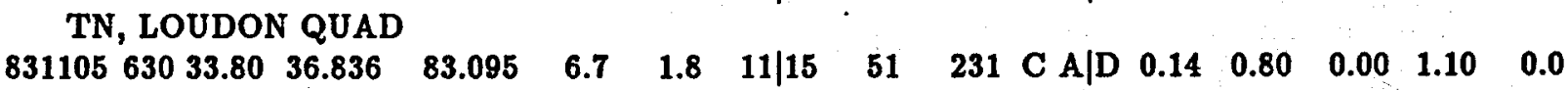
VA, PENNINGTON GAP QUAD; VA/KY BORDER

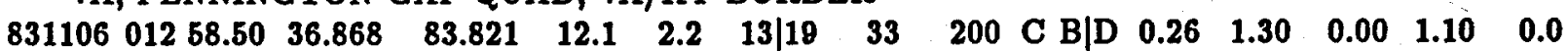
KY, FOUNT QUAD

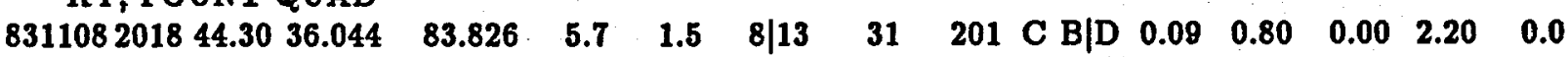
TN, JOHN SEVIER QUAD 
DATE ORGIN LAT ${ }^{\circ} \mathrm{N}$ LONG ${ }^{\circ} \mathrm{W}$ DEP MAG ST|PH DMIN GAP Q SQD RMS ERH1 ERH2 ERZ AZM

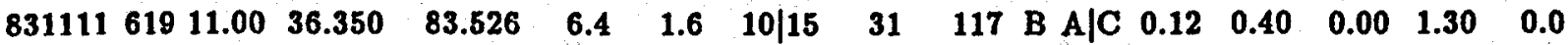
TN, DUTCH VALLEY QUAD

$831114163425.1035 .759 \quad 83.969$

TN, MARYVILLE QUAD

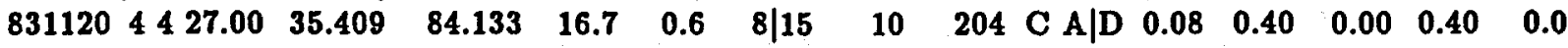

TN, RAFTER QUAD

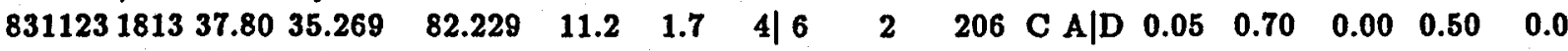

NC, LAKE LURE QUAD

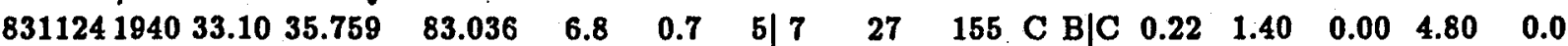

TN, WATERVILLE QUAD; TN/NC BORDER

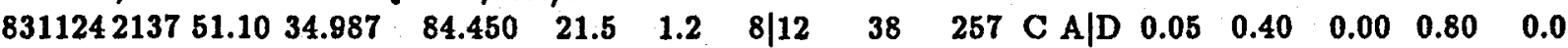

GA, EPWORTH QUAD; GA/TN BORDER

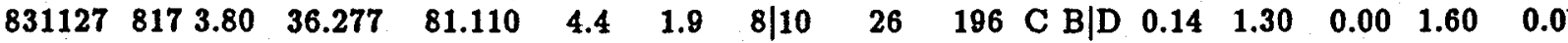

NC, TRAPHILL QUAD

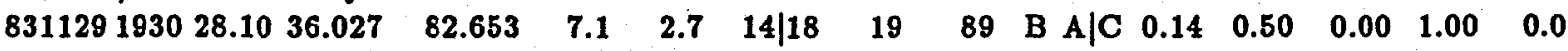

TN, GREYSTONE QUAD

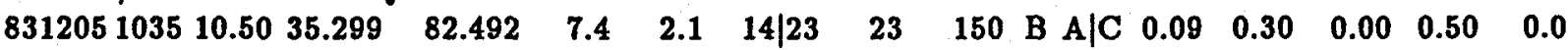

NC, HENDERSONVILLE QUAD

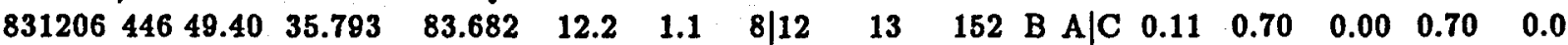

TN, WALDEN CREEK QUAD

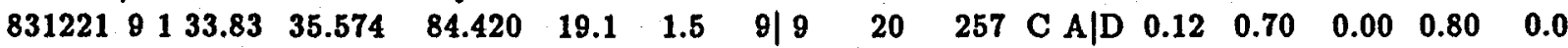

TN, MADISONVILLE QUAD

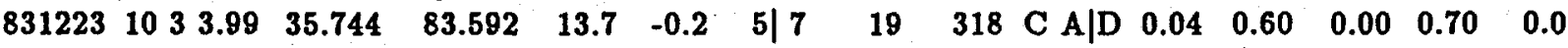
TN, GATLINBURG QUAD

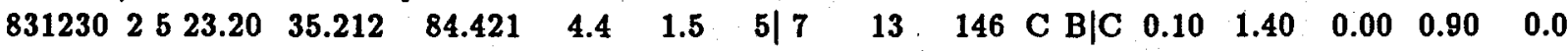
TN, MC EARLAND QUAD

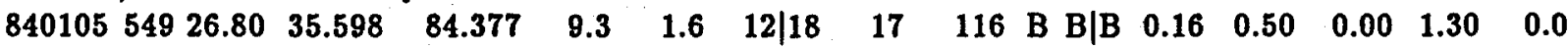
TN, SWEETWATER QUAD

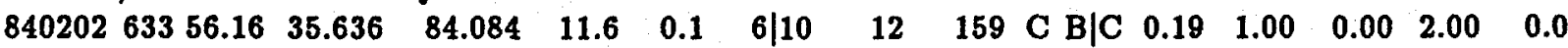
TN, BINFIELD QUAD

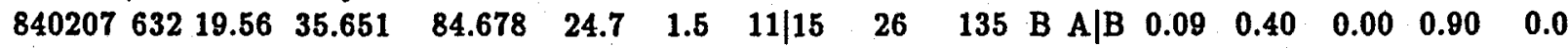
TN, TEN MILE QUAD

$\begin{array}{llllllllllllllllll}840214 & 1340 & 8.79 & 35.309 & 82.489 & 7.8 & 1.8 & 16 \mid 20 & 23 & 169 & \text { B A } & \text { A C } & 0.12 & 0.40 & 0.00 & 0.80 & 0.0\end{array}$ NC, HENDERSONVILLE QUAD

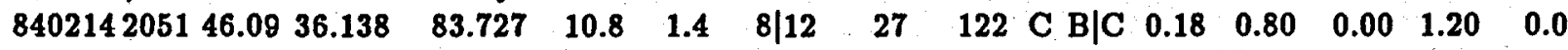
TN, LUTTRELL QUAD; FORESHOCK OF 20:54 EVENT

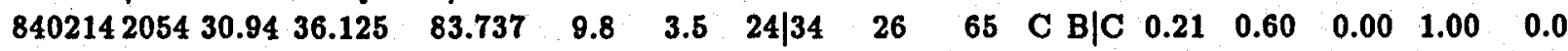
TN, LUTTRELL QUAD; FELT KNOXVILLE AREA

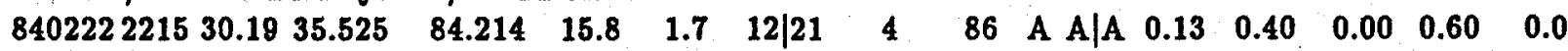
TN, VONORE QUAD

$\begin{array}{llllllllllllllllll}840227 & 17 & 8 & 11.01 & 34.662 & 85.390 & 10.8 & 2.1 & 10 \mid 14 & 35 & 159 & \text { B A A C } & 0.08 & 0.30 & 0.00 & 0.40 & 0.0\end{array}$ GA, CEDAR GROVE QUAD

$\begin{array}{llllllllllllllllll}840304 & 11 & 6 & 27.94 & 36.042 & 83.657 & 11.0 & 1.3 & 4 \mid 7 & 44 & 192 & \text { D C } & \text { C D } & 0.13 & 2.20 & 0.00 & 5.80 & 0.0\end{array}$ TN, MASCOT QUAD

$\begin{array}{llllllllllllllll}840311 & 6548.44 & 35.587 & 84.161 & 14.1 & -0.9 & 9 \mid 14 & 10 & 110 & \text { B } & \text { A|B } & 0.10 & 0.40 & 0.00 & 0.60 & 0.0\end{array}$ TN, VONORE QUAD

$\begin{array}{lllllllllllllllll}840314 & 132 & 52.29 & 35.527 & 84.254 & 18.9 & -1.2 & 7 \mid 11 & 16 & 155 & \text { B A|C } & 0.07 & 0.40 & 0.00 & 0.70 & 0.0\end{array}$ TN, MADISONVILLE QUAD 
DATE ORGIN LAT $^{\circ} \mathrm{N}$ LONG ${ }^{\circ} W$ DEP MAG ST/PH DMIN GAP Q SQD RMS ERH1 ERH2 ERZ AZM

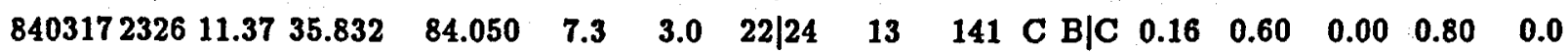
TN, LOUISVILLE QUAD; FELT KNOXVILLE AREA

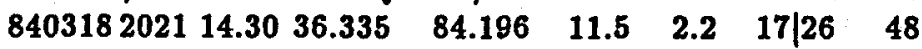
TN, JACKSBORO QUAD

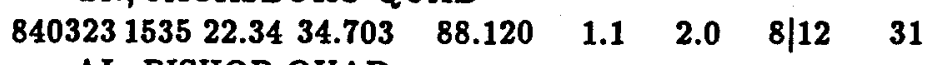
AL, BISHOP QUAD

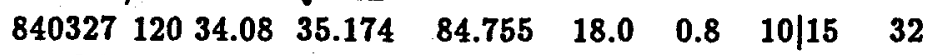
TN, EAST CLEVELAND QUAD

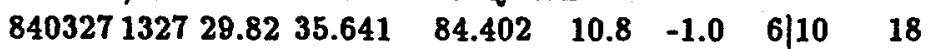
TN, PHILADELPHIA QUAD

$84041674011.68 \quad 34.696 \quad 85.062$ GA, VILLANOW QUAD

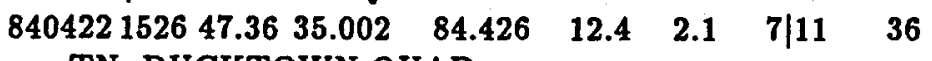
TN, DUCKTOWN QUAD

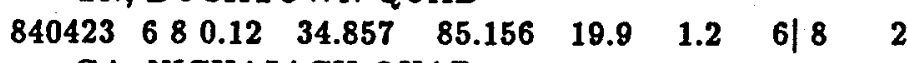
GA, NICKAJACK QUAD

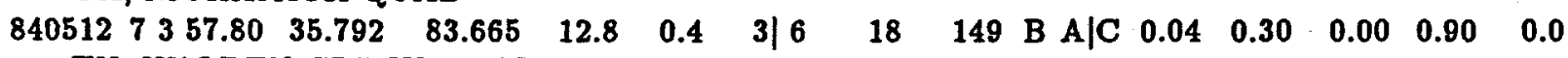
TN, WALDEN CREEK QUAD

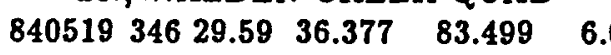
TN, HOWARD QUARTER QUAD

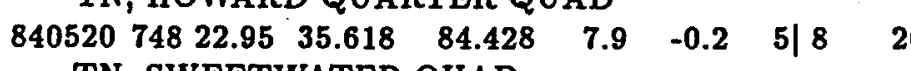
TN, SWEETWATER QUAD

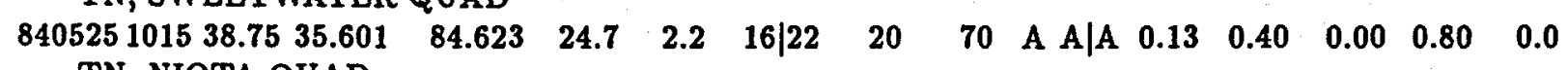
TN, NIOTA QUAD

$\begin{array}{llllllllllllllll}840602 & 912 & 38.22 & 35.925 & 83.839 & 13.6 & -0.4 & 4 \mid 6 & 4 & 258 & \text { C A A D } & 0.04 & 0.50 & 0.00 & 0.50 & 0.0\end{array}$ TN, SHOOKS GAP QUAD

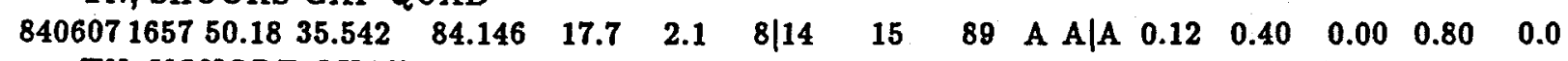
TN, VONORE QUAD

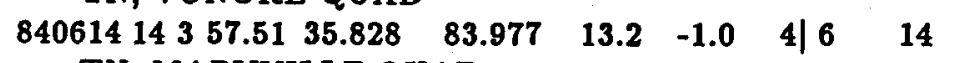
TN, MARYVILLE QUAD

\begin{tabular}{l}
$84062314357.26 \quad 35.787 \quad 83.993 \quad 13.8$ \\
\hline
\end{tabular} TN, MARYVILLE QUAD

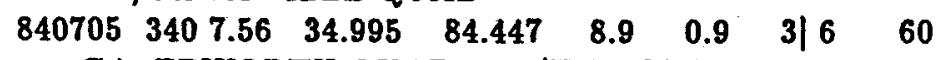
GA, EPWORTH QUAD; GA/TN BORDER

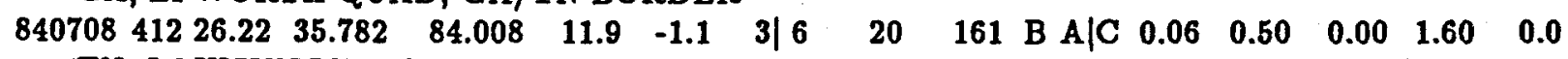
TN, LOUISVILLE QUAD

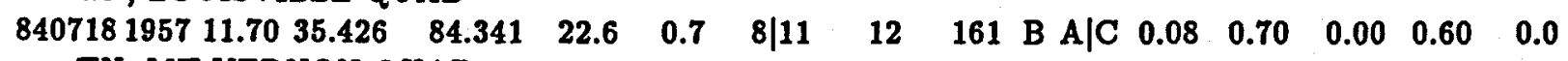
TN, MT VERNON QUAD

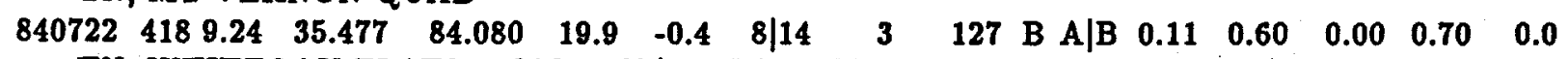
TN, WHITEOAK FLATS QUAD; TN/NC BORDER

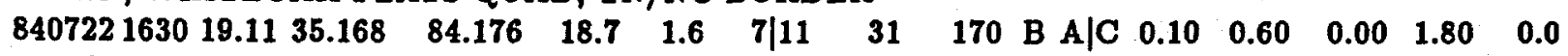
NC, UNAKA QUAD; NC/TN BORDER

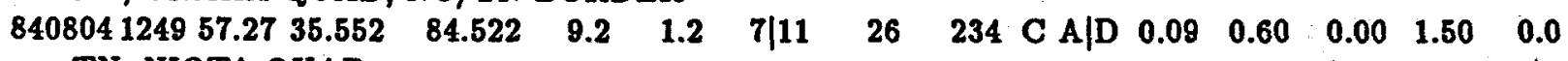
TN, NIOTA QUAD

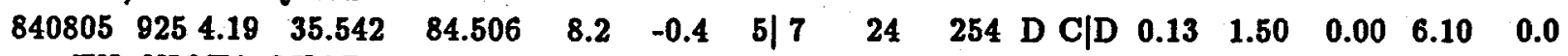
TN, NIOTA QUAD

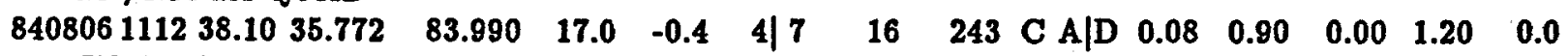
TN, MARYVILLE QUAD; FORESHOCK OF 11:13 M1.3 EVENT 
DATE ORGIN LAT $^{\circ} \mathrm{N}$ LONG ${ }^{\circ} \mathrm{W}$ DEP MAG ST|PH DMIN GAP Q SQD RMS ERH1 ERH2 ERZ AZM

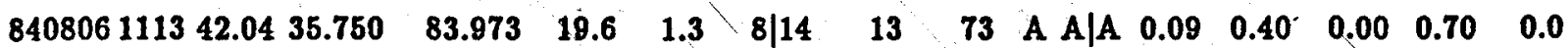
TN, MARYVILLE QUAD

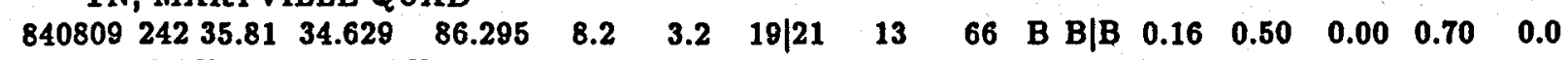
AL, PAINT ROCK QUAD

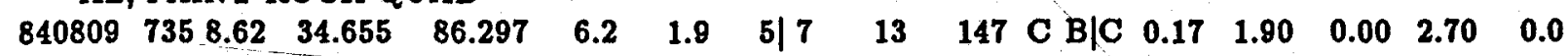
AL, PAINT ROCK QUAD; AFTERSHOCK OF 02:42 M3.2 EVENT

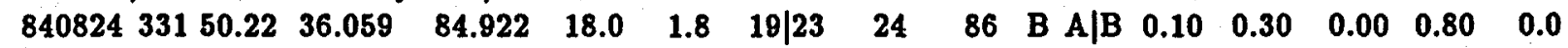
TN, FOX CREEK QUAD

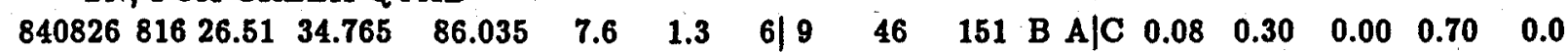
AL, MUD CREEK QUAD

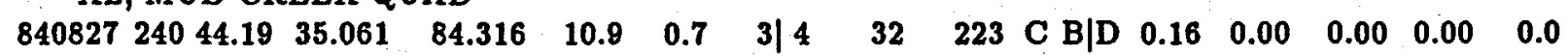
TN, ISABELLA QUAD; TN/NC BORDER

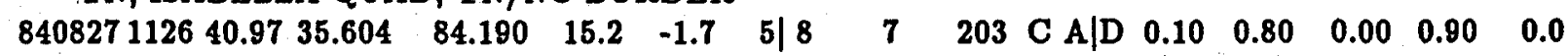
TN, VONORE QUAD

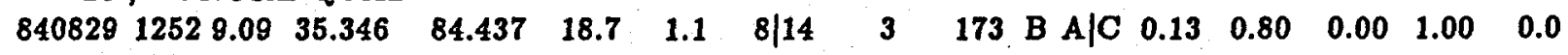
$\begin{array}{rllllllllllllll}\text { TN, MECCA } & \text { QUAD } & & & & & & & & & & & & & \end{array}$ TN, MADISONVILLE QUAD

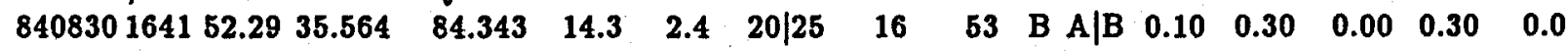
TN, MADISONVILLE QUA; AFTERSHOCK OF 16:26 M3.1 EVENT

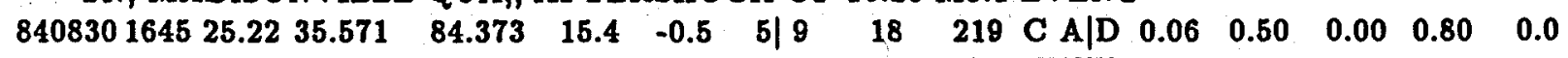
TN, MADISONVILLE QUAD; AFTERSHOCK OF 16:26 M3.1 EVENT

$\begin{array}{llllllllllllllllll}8409041459 & 35.00 & 35.313 & 83.598 & 5.0 & 1.5 & 7 \mid 12 & 39 & 224 & \text { C A } \mid \text { D } & 0.09 & 0.80 & 0.00 & 1.60 & 0.0\end{array}$ NC, WESSER QUAD

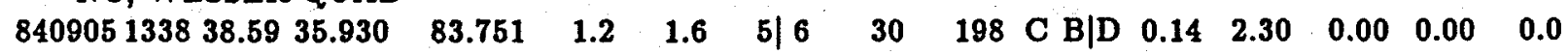
TN, SHOOKS GAP QUAD

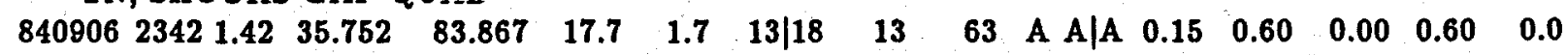
TN, WILDWOOD QUAD

$\begin{array}{llllllllllllllll}840923 & 1018 & 1.27 & 35.538 & 84.162 & 10.4 & 0.3 & 4 \mid 7 & 8 & 157 & \text { B A } \mid C & 0.04 & 0.30 & 0.00 & 0.50 & 0.0\end{array}$ TN, VONORE QUAD

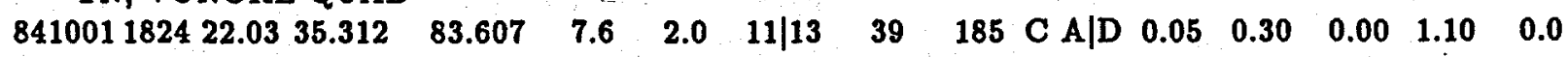
$\begin{array}{llllllllllllll}\text { NC, WESSER QUAD } & & & & & & & & \end{array}$

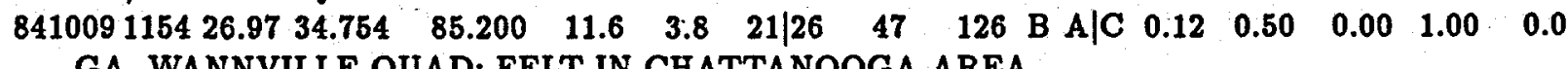
GA, WANNVILLE QUAD; FELT IN CHATTANOOGA AREA

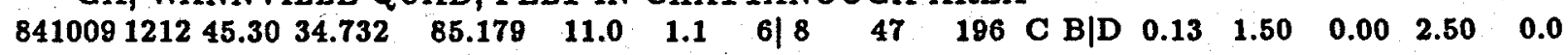
GA, CATLETT QUAD; AFTERSHOCK OF 11:54 EVENT

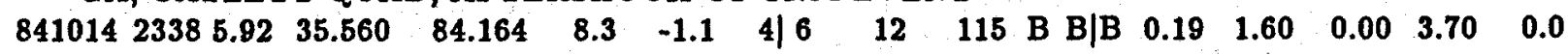
TN, VONORE QUAD

$\begin{array}{lllllllllllllllll}841015 & 1656 & 52.47 & 34.751 & 85.203 & 9.5 & 1.8 & 13 \mid 19 & 3 & 108 & \text { B A A B } & 0.11 & 0.40 & 0.00 & 0.30 & 0.0\end{array}$ GA, WANNVILLE QUAD; AFTERSHOCK OF 10/09 (11:54) M3.8 EVENT

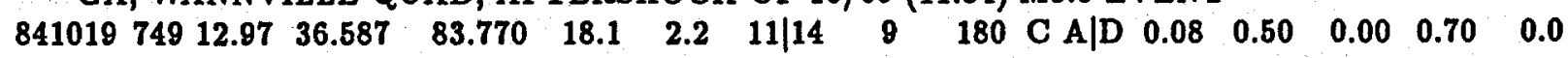
TN, FORK RIDGE QUAD; TN/KY BORDER

$\begin{array}{llllllllllllllllll}841022 & 1858 & 41.77 & 36.364 & 81.677 & 10.7 & 3.2 & 11 \mid 12 & 41 & 202 & \text { C A A D } & 0.10 & 0.50 & 0.00 & 0.60 & 0.0\end{array}$ NC, ZIONVILLE QUAD; NC/TN BORDER, FELT IN BOONE,NC AND AREA

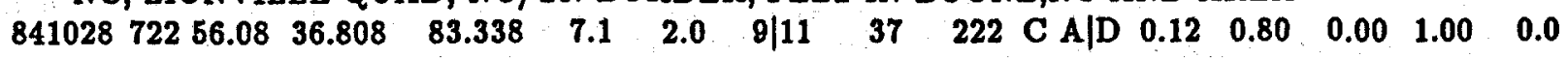
KY, HARLAN QUAD

$\begin{array}{llllllllllllllll}841029 & 939 & 48.82 & 34.828 & 84.936 & 13.3 & 1.5 & 7 \mid 10 & 41 & 155 & \text { B A A C } & 0.08 & 0.50 & 0.00 & 0.80 & 0.0\end{array}$ GA, DALTON NORTH QUAD 
DATE ORGIN LAT $^{\circ} \mathrm{N}$ LONG ${ }^{\circ} \mathrm{W}$ DEP MAG ST|PH DMIN GAP Q SQD RMS ERH1 ERH2 ERZ AZM $\begin{array}{lllllllllllllll}8411031138 & 17.07 & 35.833 & 83.773 & 12.6 & -0.2 & 7 \mid 11 & 9 & 122 & \text { B A|B } & 0.12 & 0.50 & 0.00 & 0.90 & 0.0\end{array}$ TN, WILDWOOD QUAD

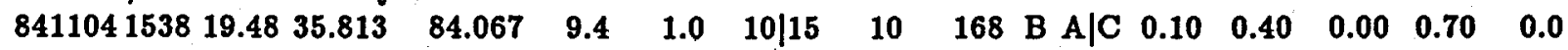
TN, LOUISVILLE QUAD

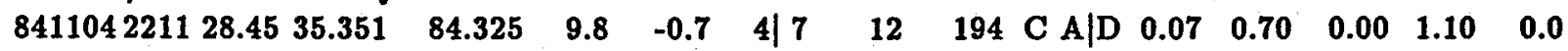
TN, TELLICO PLAINS QUAD

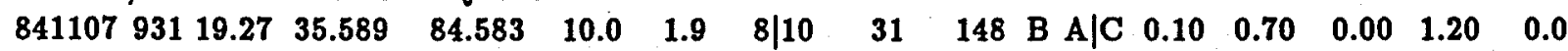
TN, NIOTA QUAD

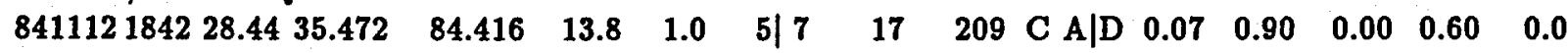
TN, ENGLEWOOD QUAD

$\begin{array}{lllllllllllllll}8411261142 & 58.93 & 35.619 & 84.264 & 8.6 & 1.4 & 6 \mid 10 & 7 & 145 \text { B A } \mid \mathrm{C} & 0.09 & 0.40 & 0.00 & 0.70 & 0.0\end{array}$ TN, MADISONVILLE QUAD

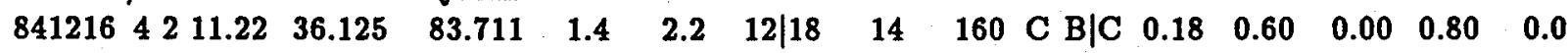
TN, LUTTRELL QUAD

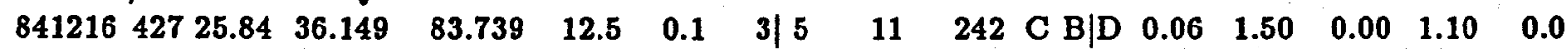
TN, LUTTRELL QUAD; AFTERSHOCK OF 04:02 EVENT

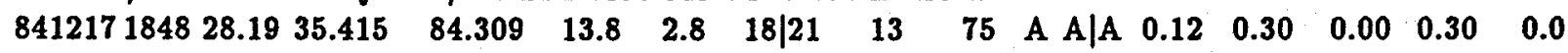
TN, MT VERNON QUAD; FORESHOCK AT 1846, Md $=-0.5$

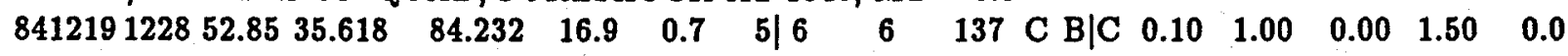
TN, VONORE QUAD

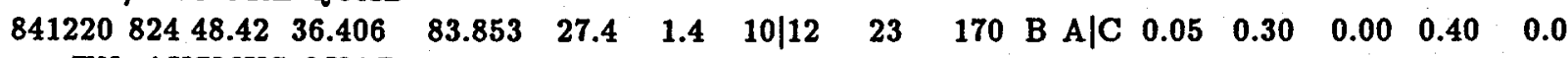
TN, AUSMUS QUAD

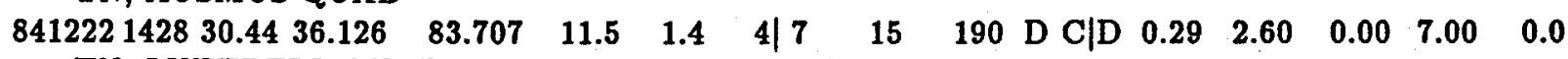
TN, LUTTRELL QUAD

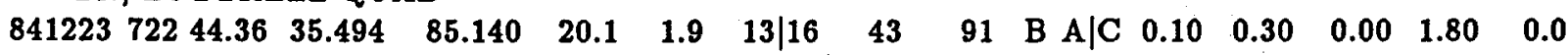
TN, BRAYTON QUAD

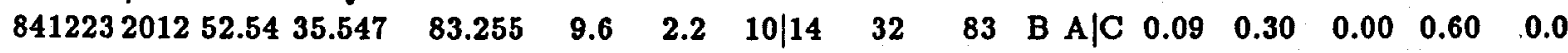
NC, SMOKEMONT QUAD; NC/TN BORDER

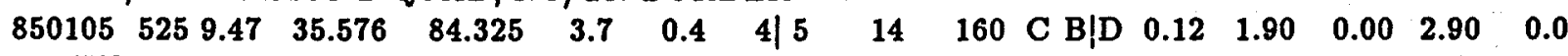
TN, MADISONVILLE QUAD

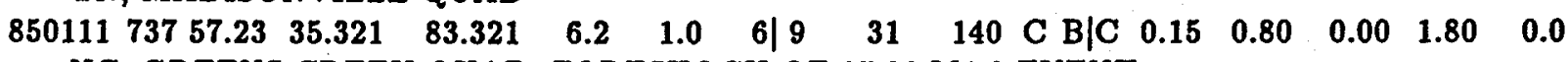
NC, GREENS CREEK QUAD; FORESHOCK OF 07:38 M1.8 EVENT

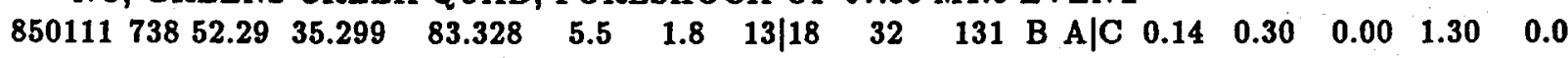
NC, GREENS CREEK QUAD

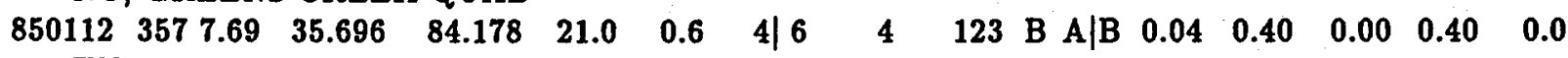
TN, MEADOW QUAD

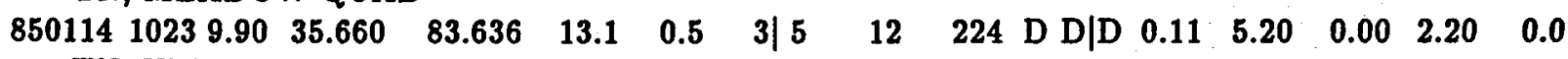
TN, WEAR COVE QUAD

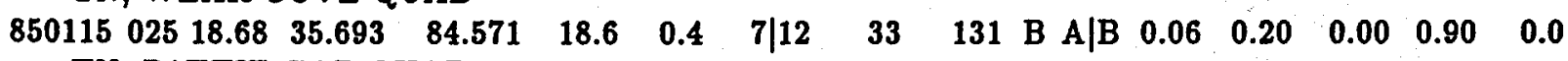
TN, PATTIE GAP QUAD

$\begin{array}{lllllllllllllll}850119 & 1859.83 & 35.686 & 84.213 & 9.8 & 0.8 & 7 \mid 10 & 2 & 83 & \text { A A A A } & 0.12 & 0.60 & 0.00 & 0.70 & 0.0\end{array}$

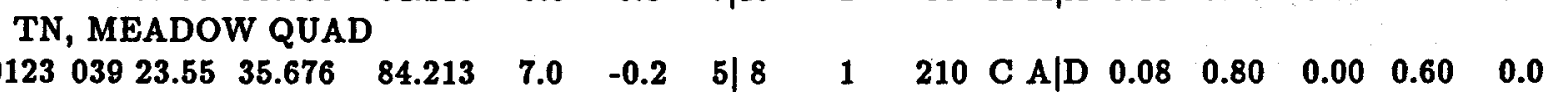

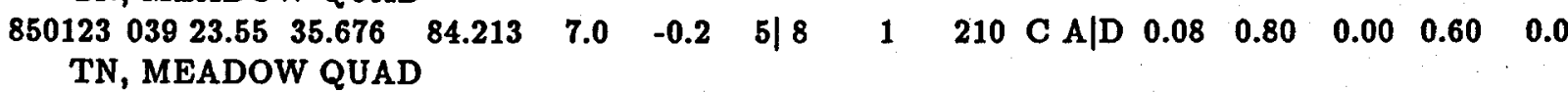

$\begin{array}{lllllllllllllllll}850123 & 039 & 23.36 & 35.688 & 84.230 & 7.8 & 0.2 & 4 & 4 & 3 & 222 & \text { C A A D } & 0.00 & 0.00 & 0.00 & 0.00 & 0.0\end{array}$ TN, MEADOW QUAD

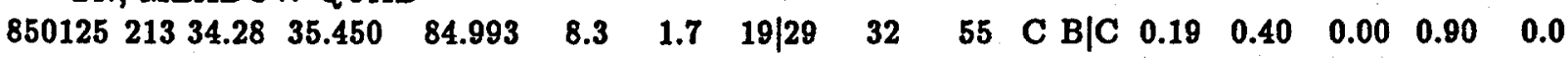
TN, BIG SPRING QUAD 
DATE ORGIN LAT ${ }^{\circ} \mathrm{N}$ LONG ${ }^{\circ} W$ DEP MAG ST|PH DMIN GAP Q SQD RMS ERH1 ERH2 ERZ AZM

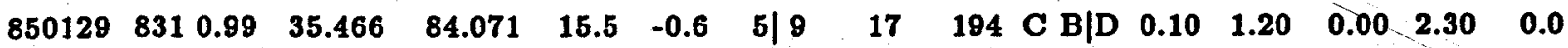
TN, WHITEOAK FLATS QUAD; TN/NC BORDER

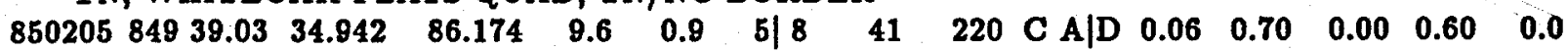
AL, ESTILL FORK QUAD; AL/TN BORDER

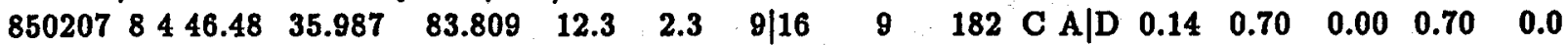
TN, SHOOKS GAP QUAD

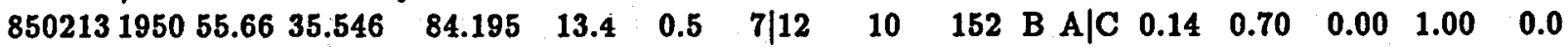
TN, VONORE QUAD

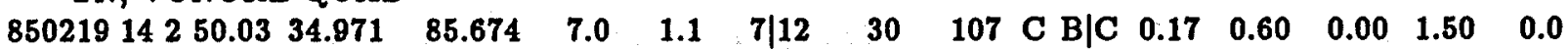
AL, BRIDGEPORT QUAD; AL/TN/GA BORDER

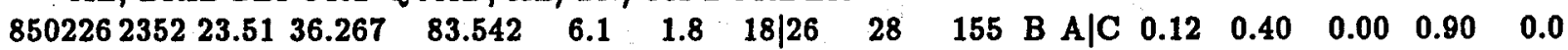
TN, DUTCH VALLEY QUAD

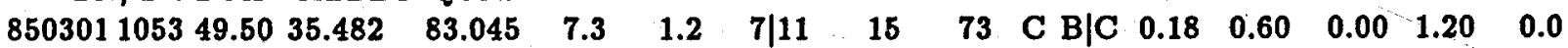
NC, HAZELWOOD QUAD

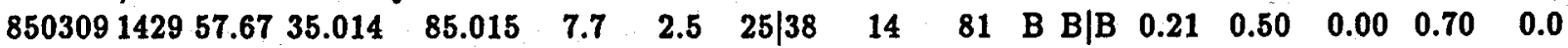
TN, OOLTEWAH QUAD; TN/GA BORDER

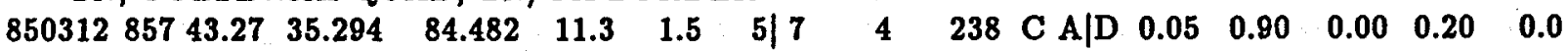
TN, MECCA QUAD

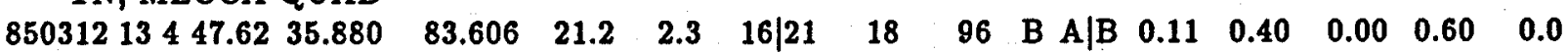
TN, DOUGLAS DAM QUAD

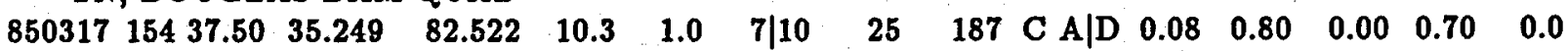
NC, STANDING STONE MTN QUAD; NC/SC BORDER

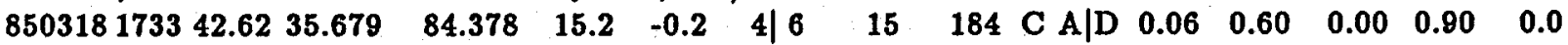
TN, PHILADELPHIA QUAD

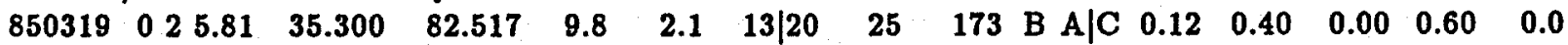
NC, HORSE SHOE QUAD; FELT IN HENDERSONVILLE, NC

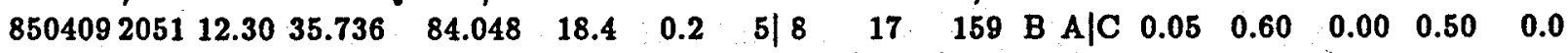
TN, BINFIELD QUAD; FORESHOCK OF 850410 (10:53) $M=2.3$ EVENT

$\begin{array}{llllllllllllllllll}850409 & 2141 & 0.66 & 35.729 & 84.036 & 16.1 & 1.4 & 9 & 17 & 17 & 112 & \text { B } & \text { A } \mid B & 0.09 & 0.30 & 0.00 & 0.50 & 0.0\end{array}$ TN, BINFIELD QUAD; FORESHOCK OF 850410 (10:53) $M=2.3$ EVENT

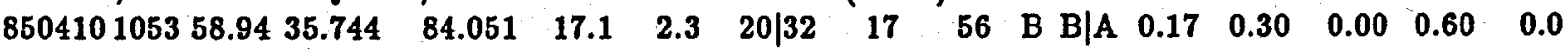
TN, BINFIELD QUAD

$\begin{array}{lllllllllllllll}8504171612 & 11.00 & 35.306 & 83.633 & 11.0 & 1.4 & 6 / 10 & 41 & 114 & \text { B A|C } & 0.14 & 0.60 & 0.00 & 1.50 & 0.0\end{array}$ NC, HEWITT QUAD

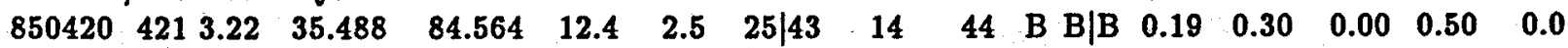
TN, ATHENS QUAD

$\begin{array}{llllllllllllllllllll}850429 & 7422.99 & 35.620 & 84.222 & 10.0 & 0.5 & 8 & 13 & 5 & 112 & \text { B } & \text { A|B } & 0.13 & 0.50 & 0.00 & 0.70 & 0.0\end{array}$ TN, VONORE QUAD

$\begin{array}{lllllllllllllllll}850507 & 1410 & 4.04 & 35.110 & 84.793 & 13.4 & 1.6 & 9 \mid 15 & 39 & 123 & \text { B } & \text { A } \mid C & 0.13 & 0.40 & 0.00 & 0.70 & 0.0\end{array}$ TN, FELKER QUAD

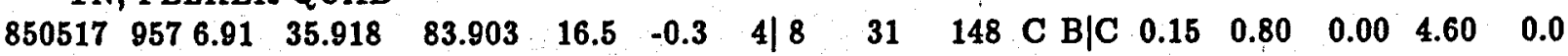
TN, KNOXVILLE QUAD

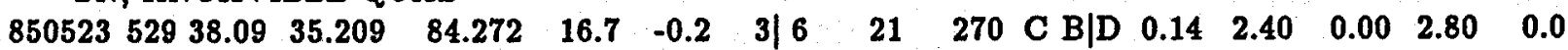
TN, FARNER QUAD; TN/NC BORDER

$\begin{array}{lllllllllllllllll}850617 & 030 & 47.81 & 35.588 & 84.161 & 19.7 & 0.3 & 11 \mid 19 & 10 & 75 & \text { A A A A } & 0.14 & 0.50 & 0.00 & 0.60 & 0.0\end{array}$ TN, VONORE QUAD

$\begin{array}{lllllllllllllllll}850617 & 039 & 16.93 & 35.584 & 84.168 & 18.0 & 0.1 & 7 \mid 10 & 10 & 79 & \text { A A A } & 0.13 & 0.70 & 0.00 & 1.10 & 0.0\end{array}$ TN, VONORE QUAD 
DATE ORGIN LAT ${ }^{\circ} \mathrm{N}$ LONG ${ }^{\circ} \mathrm{W}$ DEP MAG ST|PH DMIN GAP Q SQD RMS ERH1 ERH2 ERZ AZM

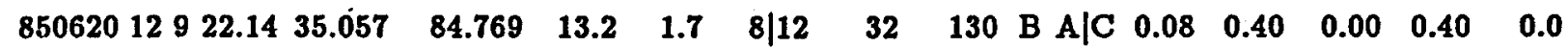
TN, FELKER QUAD

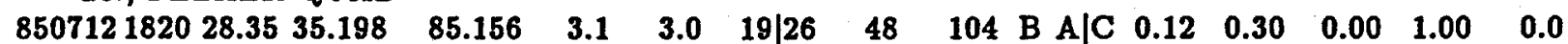
TN, DAISY QUAD; FELT AT SEQUOYAH NUCLEAR PLANT

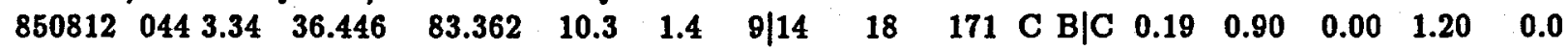
TN, SWAN ISLAND QUAD

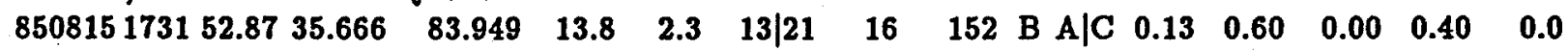
TN, BLOCKHOUSE QUAD

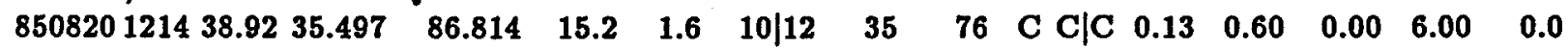
TN, LEWISBURG QUAD

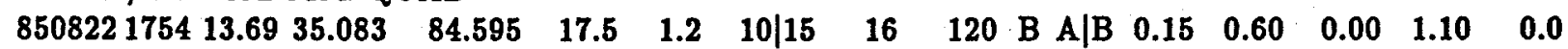
TN, CANEY CREEK QUAD

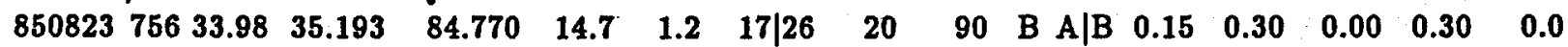
TN, EAST CLEVELAND QUAD

$\begin{array}{llllllllllllllll}850825 & 152 & 46.90 & 36.098 & 83.699 & 6.0 & 1.7 & 19 \mid 26 & 17 & 53 & \text { B A|C } & 0.11 & 0.30 & 0.00 & 0.80 & 0.0\end{array}$ TN, MASCOT QUAD

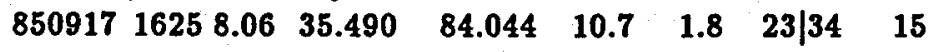
TN, WHITEOAK FLATS QUAD; TN/NC BORDER

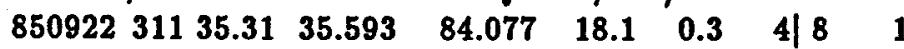
TN, TALLASSEE QUAD

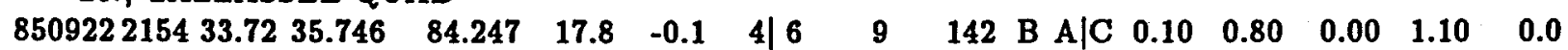
TN, MEADOW QUAD

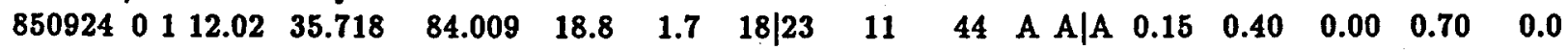
TN, BINFIELD QUAD

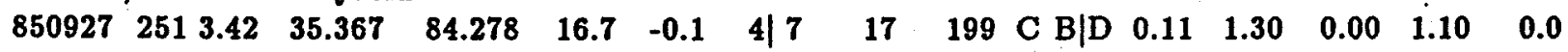
TN, TELLICO PLAINS QUAD

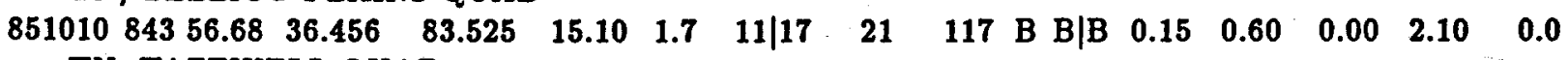
TN, TAZEWELL QUAD

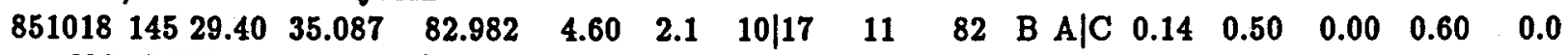
NC, REID QUAD; NC/SC BORDER

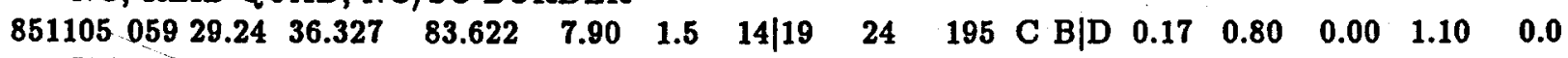
TN, DUTCH VALLEY QUAD

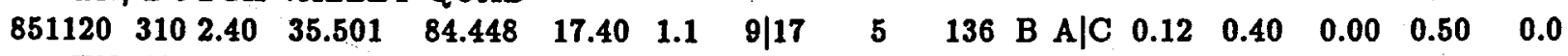
TN, SWEETWATER QUAD

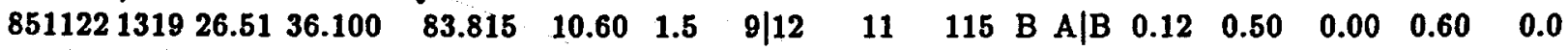
TN, JOHN SEVIER QUAD

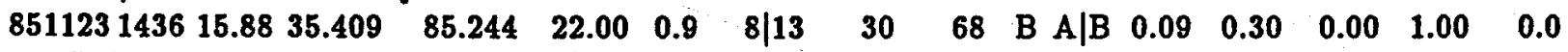
TN, BRAYTON QUAD

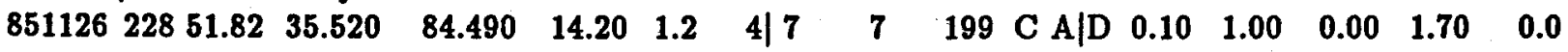
TN, SWEETWATER QUAD

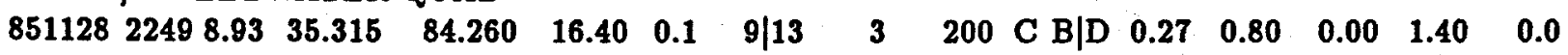
TN, TELLICO PLAINS QUAD

$\begin{array}{llllllllllllllll}851208 & 445 & 58.06 & 35.787 & 84.047 & 16.70 & 1.5 & 10 \mid 17 & 20 & 82 & \text { B A|B } & 0.14 & 0.40 & 0.00 & 0.90 & 0.0\end{array}$ TN, LOUISVILLE QUAD

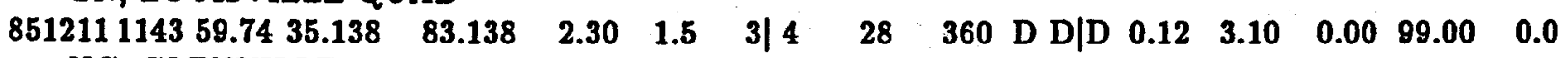
NC, GLENVILLE QUAD

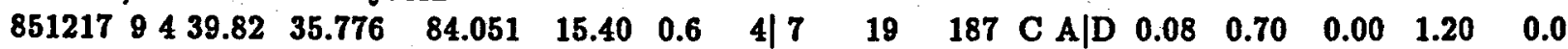
TN, LOUISVILLE QUAD 
DATE ORGIN LAT ${ }^{\circ}$ LONG ${ }^{\circ} W$ DEP MAG ST|PH DMIN GAP Q SQD RMS ERH1 ERH2 ERZ AZM

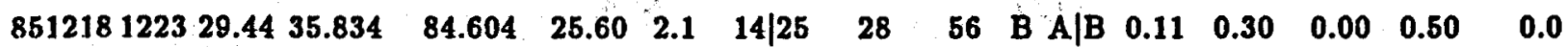
TN, BACON GAP QUAD

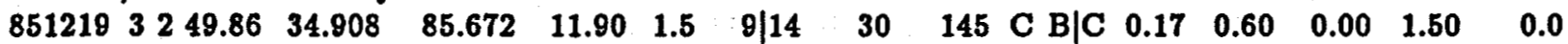
AL, BRIDGEPORT QUAD; AL/TN/GA BORDER

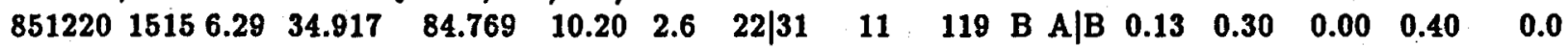
GA, BEAVERDALE QUAD; GA/TN BORDER

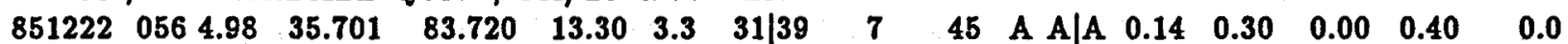
TN, WEAR COVE QUAD; FELT IN GATLINBURG AND AREA.

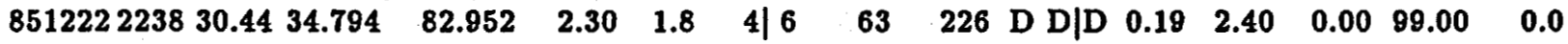
SC, OLD PICKENS QUAD

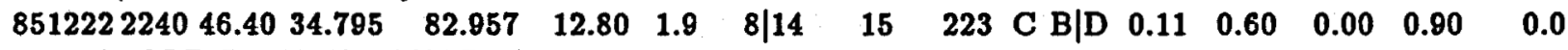
SC, OLD PICKENS QUAD

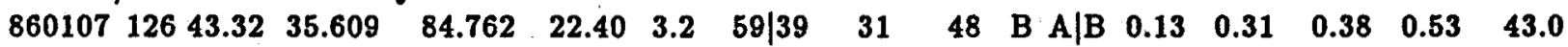
TN, DECATUR QUAD; FELT STRONGLY AT DECATUR, TN.

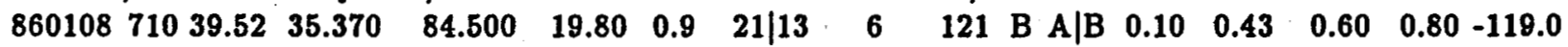
TN, ETOWAH QUAD

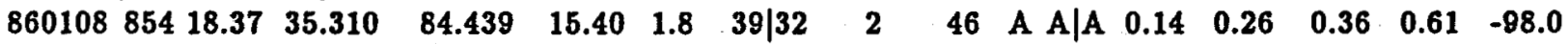

TN, MECCA QUAD

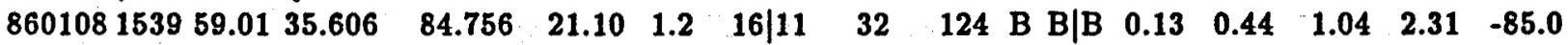
TN, DECATUR QUAD

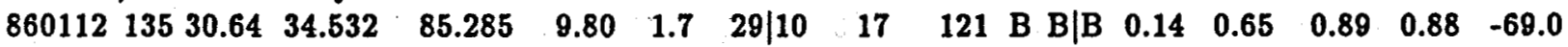
GA, TRION QUAD

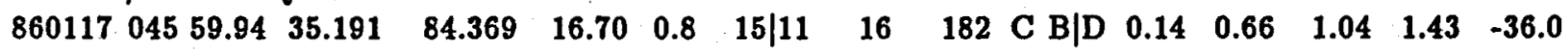
TN, FARNER QUAD; TN/NC BORDER

$\begin{array}{llllllllllllllll}860127 & 644 & 26.77 & 35.926 & 83.636 & 20.10 & 2.7 & 46 \mid 24 & 21 & 86 & \text { B A|B } & 0.14 & 0.45 & 0.50 & 0.55 & -73.0\end{array}$ TN, BOYDS CREEK QUAD

$\begin{array}{llllllllllllllll}860127 & 646 & 0.45 & 35.916 & 83.623 & 14.70 & 2.2 & 20 \mid 12 & 20 & 186 & \text { C B|D } & 0.10 & 0.59 & 1.11 & 1.90 & -91.0\end{array}$ TN, DOUGLAS DAM QUAD

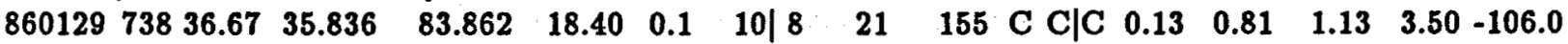
TN, WILDWOOD QUAD

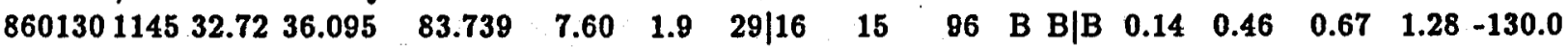
TN, MASCOT QUAD

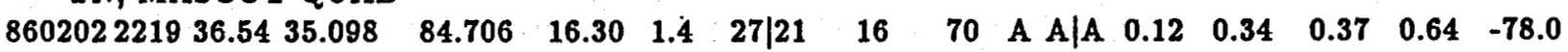
TN, PARKSVILLE QUAD

$\begin{array}{llllllllllllllll}860203 & 053 & 6.78 & 35.927 & 83.634 & 19.40 & 1.5 & 14 \mid 13 & 21 & 86 & \text { B A A } & 0.12 & 0.54 & 0.61 & 0.97 & 12.0\end{array}$ TN, BOYDS CREEK QUAD

$\begin{array}{lllllllllllllllll}860208 & 3 & 9 & 18.89 & 35.339 & 85.253 & 22.30 & 1.1 & 26 \mid 19 & 27 & 68 & \text { B A|B } & 0.12 & 0.31 & 0.44 & 0.85 & -51.0\end{array}$ TN, HENSON GAP QUAD

$\begin{array}{llllllllllllllll}860213 & 1135 & 45.45 & 34.793 & 82.907 & 4.80 & 3.5 & 35 \mid 16 & 63 & 106 & \text { C B } \mid D & 0.12 & 0.53 & 0.70 & 2.00 & -17.0\end{array}$ SC, OLD PICKENS QUAD; FELT AT DUKE POWER, NEAR CLEMSON, SC.

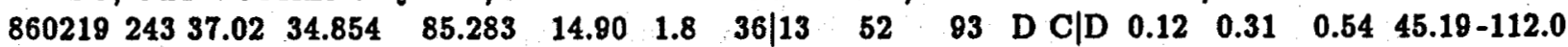
GA, KENSINGTON QUAD

$\begin{array}{llllllllllllllll}860219 & 757 & 11.28 & 35.833 & 84.105 & 11.40 & 1.5 & 32 \mid 17 & 20 & 85 & \text { B A|B } & 0.13 & 0.39 & 0.48 & 0.52 & -93.0\end{array}$ TN, LOUISVILLE QUAD

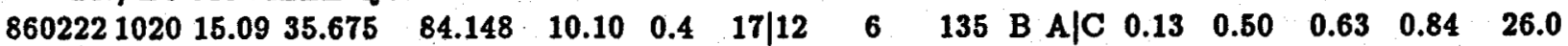
TN, MEADOW QUAD 
DATE ORGIN LAT $^{\circ} \mathrm{N}$ LONG ${ }^{\circ} \mathrm{W}$ DEP MAG ST|PH DMIN GAP Q SQD RMS ERH 1 ERH2 ERZ AZM

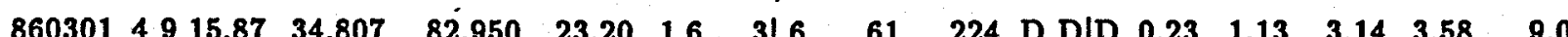
SC, OLD PICKENS QUAD

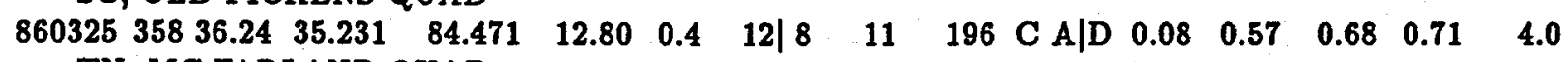
TN, MC FARLAND QUAD $\begin{array}{rrr}860325 & 445 \quad 34.08 \quad 35.244 & 84.466\end{array}$ TN, MC FARLAND QUAD

860411 6 $525.86 \quad 34.676 \quad 85.430$
GA, CEDAR GROVE QUAD $860413231620.0535 .515 \quad 84.191$ TN, VONORE QUAD

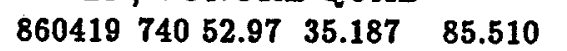
TN, WHITWELL QUAD

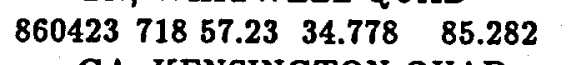
GA, KENSINGTON QUAD

$860427223026.4435 .919 \quad 83.729$ TN, BOYDS CREEK QUAD

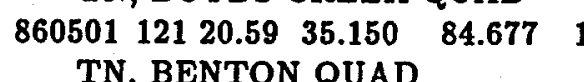

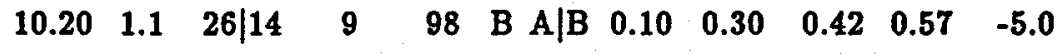
$\begin{array}{llllllllllll}13.00 & 0.0 & 9 / 12 & 7 & 180 & \text { C B } & \text { B|D } & 0.11 & 0.87 & 1.76 & 0.76 & 25.0\end{array}$ TN, BENTON QUAD

$860504192540.18 \quad 35.510 \quad 84.201$ TN, VONORE QUAD $\begin{array}{rr}860508 \quad 849.48 \quad 35.383 & 85.765 \\ \text { TN, CANE HOLLOW QUAD }\end{array}$

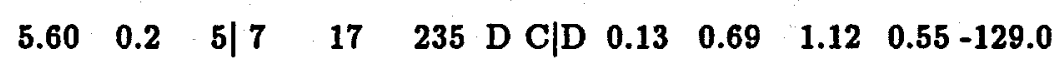
$\begin{array}{lllllllllllll}27.30 & 3.0 & 28 & 56 & 28 & 74 & \text { B } & \text { A|B } & 0.11 & 0.31 & 0.44 & 0.78 & -40.0\end{array}$ $\begin{array}{llllllllllll}14.80 & 1.9 & 33 \mid 46 & 23 & 140 & \text { B } & \text { A } \mid C & 0.13 & 0.28 & 0.36 & 0.88 & -133.0\end{array}$ $\begin{array}{lllllllllllll}19.70 & 2.1 & 22 & 26 & 14 & 72 & \text { A } & \text { A } \mid \text { A } & 0.12 & 0.32 & 0.39 & 0.64 & -6.0\end{array}$ 860513143035.9735 .539
TN, VONORE QUAD

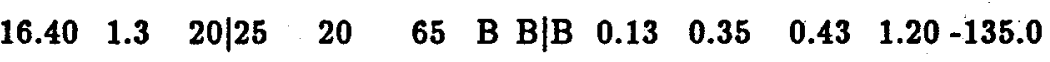

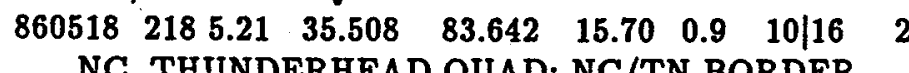
NC, THUNDERHEAD QUAD; NC/TN BORDER

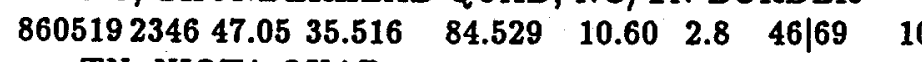
TN, NIOTA QUAD

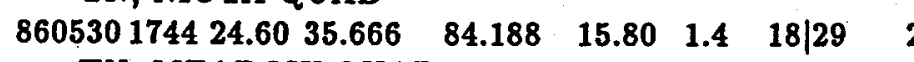
TN, MEADOW QUAD

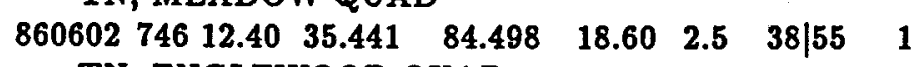
TN, ENGLEWOOD QUAD

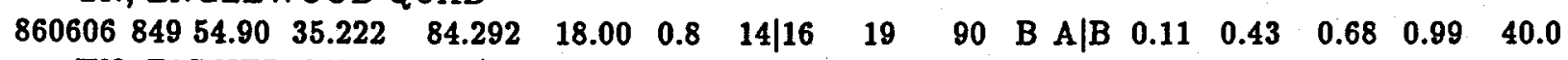
TN, FARNER QUAD; TN/NC BORDER

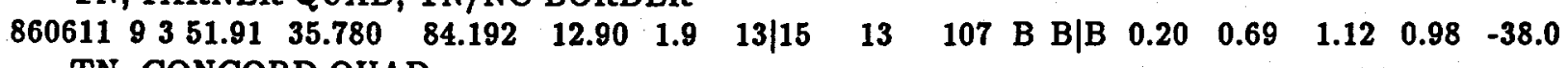
TN, CONCORD QUAD

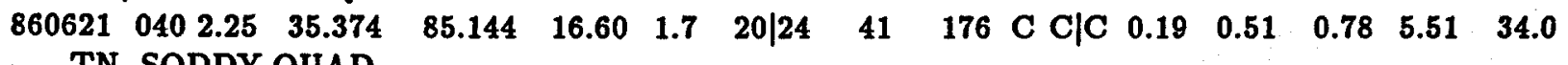
TN, SODDY QUAD $\begin{array}{ccccccccccccccc}8606241922 & 42.03 & 35.990 & 83.931 & 24.30 & 2.9 & 40 \mid 47 & 8 & 59 & \text { A A|A } & 0.15 & 0.30 & 0.35 & 0.57 & 16.0 \\ \text { TN, KNOXVILLE QUAD } & & & & & & & & \end{array}$

$\begin{array}{lllllllllllllll}8606242254 & 46.53 & 35.986 & 83.931 & 24.10 & 1.8 & 29 \mid 32 & 8 & 59 & \text { A A A A } & 0.14 & 0.33 & 0.46 & 0.49 & 7.0\end{array}$ TN, KNOXVILLE QUAD

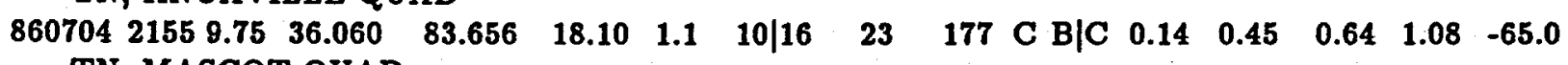
TN, MASCOT QUAD

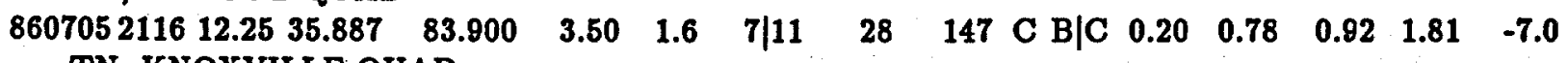
TN, KNOXVILLE QUAD . 
DATE ORGIN LAT $^{\circ} N$ LONG ${ }^{\circ} W$ DEP MAG ST|PH DMIN GAP Q SQD RMS ERH1 ERH2 ERZ AZM

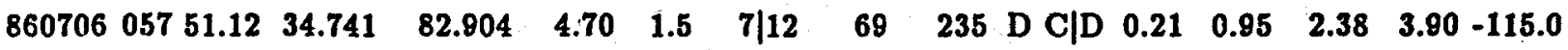
SC, SENECA QUAD; RESERVOIR ASSOCIATED

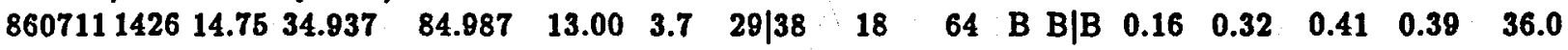
GA, COHUTTA QUAD; GA/TN BORDER, WIDELY FELT THROUGHOUT AREA WITH MINOR DAMAGE

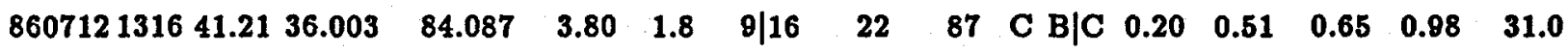
TN, POWELL QUAD

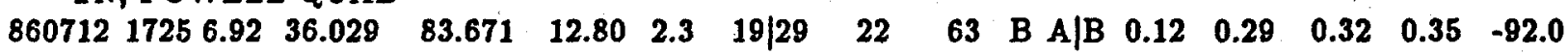
TN, MASCOT QUAD

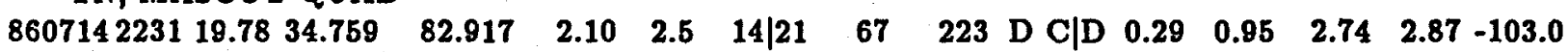
SC, OLD PICKENS QUAD; RESERVOIR ASSOCIATED SEQUENCE BEGINNING JUNE 11, $1886^{*}$.

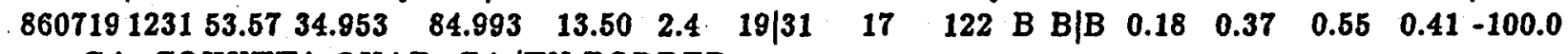
GA, COHUTTA QUAD; GA/TN BORDER

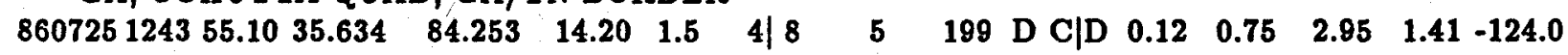
TN, LOUDON QUAD

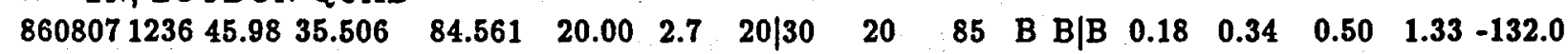
TN, NIOTA QUAD

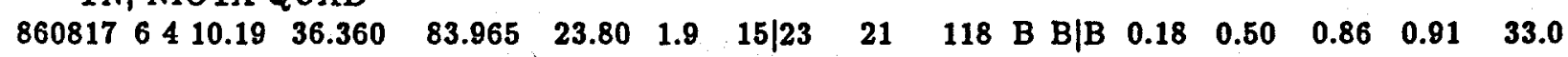
TN, WHITE HOLLOW QUAD

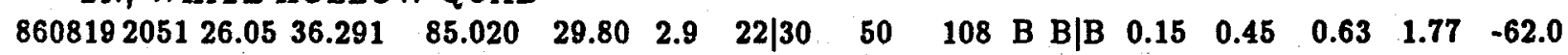
TN, WILDER QUAD; FELT IN JAMESTOWN, TN AREA

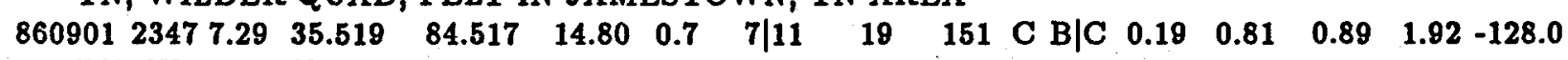
TN, NIOTA QUAD

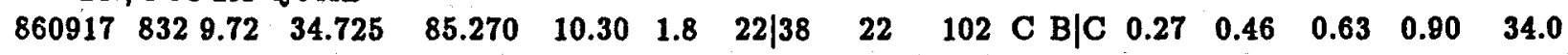
GA, ESTELLE QUAD

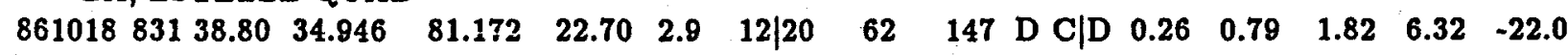
SC, ROCK HILL QUAD; FELT IN ROCK HILL, SC

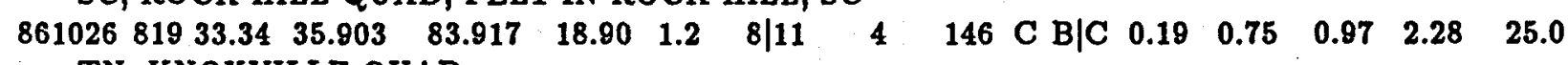
TN, KNOXVILLE QUAD

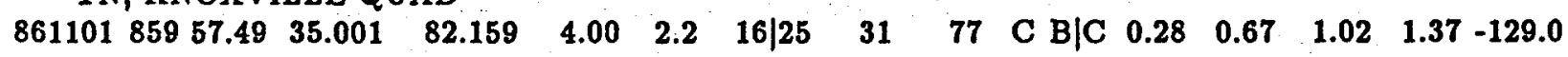
SC, INMAN QUAD

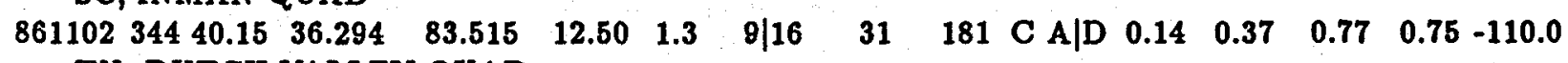
TN, DUTCH VALLEY QUAD

$86110217447.12 \cdot 35.761 \quad 84.191$ TN, CONCORD QUAD

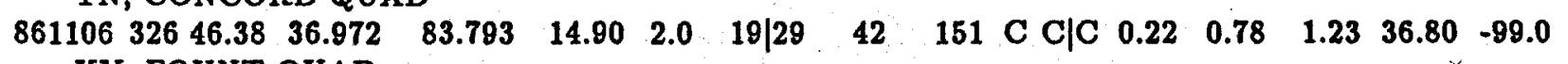
KY, FOUNT QUAD

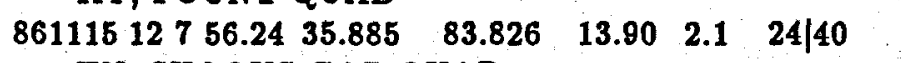
TN, SHOOKS GAP QUAD

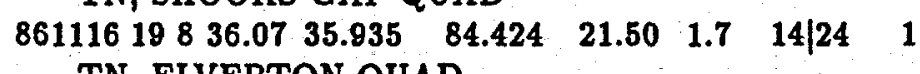
TN, ELVERTON QUAD

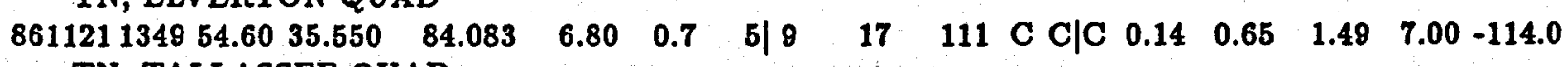
TN, TALLASSEE QUAD

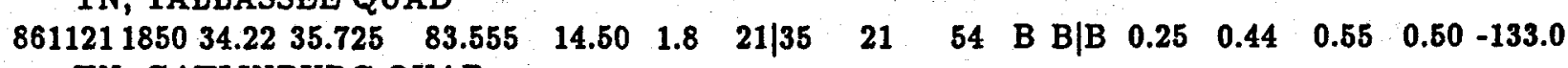
TN, GATLINBURG QUAD

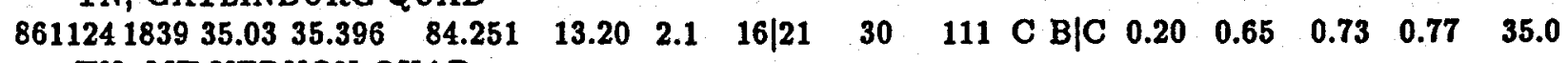
TN, MT VERNON QUAD

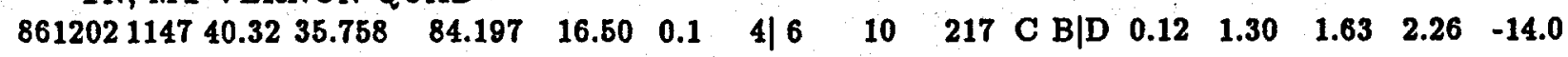
TN, CONCORD QUAD 
DATE ORGIN LAT ${ }^{\circ} N$ LONG ${ }^{\circ} W$ DEP MAG ST|PH DMIN GAP Q SQD RMS ERH1 ERH2 ERZ AZM

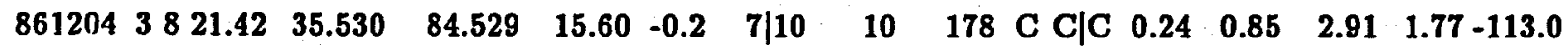
TN, NIOTA QUAD

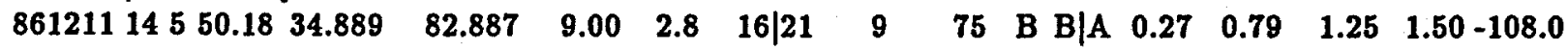
SC, SALEM QUAD; FELT IN SALEM, SC; RESERVOIR ASSOCIATED (LAKE KEOWEE)

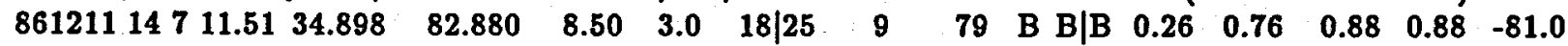
SC, SALEM QUAD; FELT IN SALEM, SC; RESERVOIR ASSOCIATED (LAKE KEOWEE)

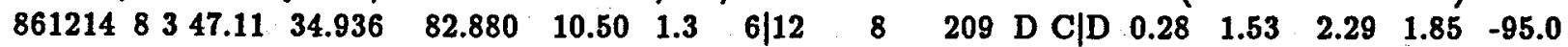
SC, SALEM QUAD; RESERVOIR ASSOCIATED (LAKE KEOWEE)

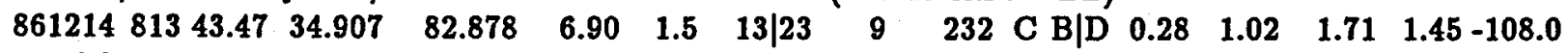
SC, SALEM QUAD; RESERVOIR ASSOCIATED (LAKE KEOWEE)

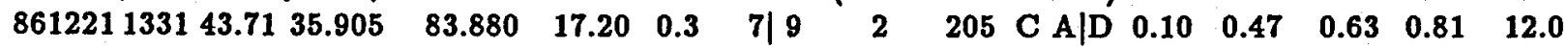
TN, KNOXVILLE QUAD

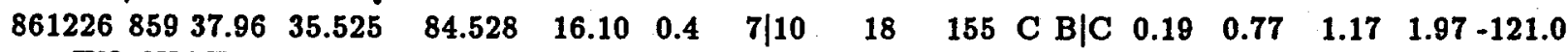
TN, NIOTA QUAD

DATE ORGIN LAT ${ }^{\circ} \mathrm{N}^{\circ} \mathrm{N}^{\circ} \mathrm{W}$ DEP MAG MOD ST|PH DMIN GAP QSQD RMS ERH1 ERH2 ERZ AZM

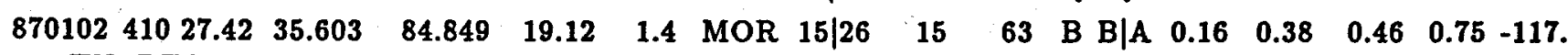
TN, DECATUR

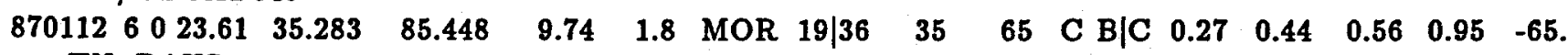
TN, DAUS

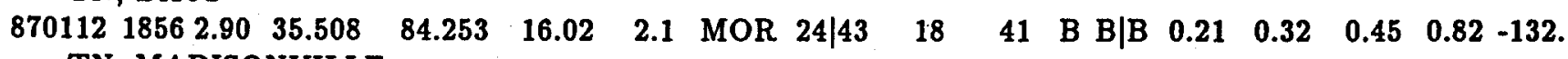
TN, MADISONVILLE

$870113105630.0935 .561 \quad 84.178$ TN, VONORE

\begin{tabular}{|c|c|c|c|c|c|c|c|c|c|c|c|c|c|}
\hline $\begin{array}{l}870116144154.9135 .845 \\
\text { TN, RODDY }\end{array}$ & 84.800 & 17.88 & 0.9 & MOR & $14 \mid 21$ & 37 & 144 & $\mathrm{C} \mathrm{B} \mid \mathrm{C}$ & 0.15 & 0.38 & 0.47 & 2.33 & -69. \\
\hline $\begin{array}{c}7011710123.46 \quad 36.253 \\
\text { TN, WHITE HOLLOV }\end{array}$ & 83.995 & 25.99 & 0.4 & MOR & $11 \mid 13$ & 15 & 310 & C A $\mid D$ & 0.11 & 0.44 & 0.57 & 0.86 & -68 . \\
\hline $\begin{array}{c}70203103236.4334 .818 \\
\text { AL, HOLLYTREE }\end{array}$ & 86.315 & 9.63 & 2.4 & MOR & $22 \mid 34$ & 25 & 70 & $\mathrm{C} \mathrm{B} \mid \mathrm{C}$ & 0.27 & 0.50 & 0.65 & 0.95 & -101 \\
\hline $\begin{array}{c}370203143127.9835 .497 \\
\text { TN, ENGLEWOOD }\end{array}$ & 84.452 & 11.52 & 1.3 & MOR & $14 \mid 20$ & 19 & 78 & B $\mathrm{B} \mid \mathrm{B}$ & 0.24 & 0.54 & 0.77 & 0.90 & - \\
\hline $\begin{array}{c}70208 \quad 1134.98 \quad 36.062 \\
\text { TN, JOHN SEVIER }\end{array}$ & 83.774 & 14.11 & 0.8 & MOR & $5 \mid 7$ & 16 & 202 & C B $\mid \mathrm{D}$ & 0.07 & 0.46 & 0.79 & 0.74 & \\
\hline $\begin{array}{c}3702109245.9834 .971 \\
\text { GA, RINGGOLD }\end{array}$ & 85.003 & 8.43 & 1.5 & MOR & $20 \mid 35$ & 16 & 91 & B $\mathrm{B} \mid \mathrm{B}$ & 0.25 & 0.41 & 0.55 & 1.08 & -113. \\
\hline $\begin{array}{c}8702118200.59 \\
\text { TN, MECCA }\end{array}$ & 84.386 & 21.48 & 0.6 & MOR & $8 \mid 14$ & 11 & 103 & B $A \mid B$ & 0.11 & 0.35 & 0.55 & 0.67 & -131. \\
\hline $\begin{array}{r}87022110142.6535 .585 \\
\text { TN, SWEETWATER }\end{array}$ & 84.430 & 15.66 & 0.7 & MOR & $9 \mid 15$ & 22 & 123 & B B|B & 0.13 & 0.38 & 0.45 & 1.12 & -132 \\
\hline $\begin{array}{c}870221222140.2635 .207 \\
\text { TN, MCFARLAND }\end{array}$ & 84.399 & 16.38 & 0.7 & MOR & $13 \mid 24$ & 14 & 90 & B B|A & 0.22 & 0.53 & 0.65 & 0.89 & \\
\hline $\begin{array}{c}870222103526.4836 .338 \\
\text { TN, JACKSBORO }\end{array}$ & 84.186 & 19.11 & 2.8 & MOR & $25 \mid 39$ & 28 & 113 & B $\mathbf{B} \mid \mathbf{B}$ & 0.28 & 0.59 & 0.74 & 2.50 & - \\
\hline $\begin{array}{l}87022381243.1635 .716 \\
\text { TN, LOUDON }\end{array}$ & 84.312 & 18.16 & 0.7 & MOR & $12 \mid 19$ & 11 & 76 & B $B \mid A$ & 0.20 & 0.53 & 0.71 & 0.89 & \\
\hline
\end{tabular}


DATE ORGIN LAT ${ }^{\circ}$ LONG ${ }^{\circ} W$ DEP MAG MOD ST|PH DMIN GAP Q SQD RMS ERH1 ERH2 ERZ AZM $\begin{array}{llllllllllllllllll}870224 & 958 & 10.59 & 34.733 & 85.333 & 9.92 & 2.4 & \text { MOR } & 20 \mid 35 & 18 & 150 & \text { C B } & \text { B C } & 0.23 & 0.51 & 0.61 & 0.74 & -99 .\end{array}$ GA, ESTELLE

$\begin{array}{llllllllllllllllll}870306 & 1030 & 40.37 & 35.998 & 84.098 & 4.99 & 1.6 & \text { MOR } & 21 \mid 33 & 21 & 164 & \text { C } & \text { B|C } & 0.22 & 0.37 & 0.54 & 0.58 & -106 .\end{array}$ TN, BEARDEN

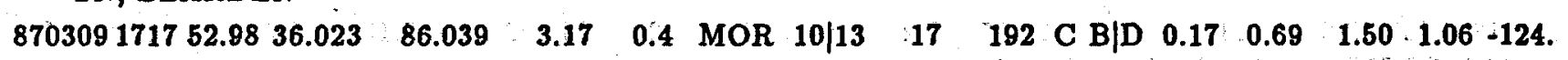
TN, ALEXANDRIA

$\begin{array}{lllllllllllllllllllll}870313 & 1955 & 22.24 & 35.665 & 84.219 & 6.74 & 1.9 & \text { MOR } & 19 \mid 33 & 1 & 53 & \text { B } & \text { B } \mid \text { A } & 0.28 & 0.48 & 0.67 & 0.67 & -118 .\end{array}$ TN, MEADOW

$\begin{array}{lllllllllllllllll}8703142041 & 58.14 & 35.778 & 84.105 & 17.30 & 1.5 & \text { MOR } & 19 \mid 34 & 16 & 63 & \text { B } & \text { B } \mid A & 0.18 & 0.31 & 0.39 & 0.65 & -105\end{array}$ TN, LOUISVILLE

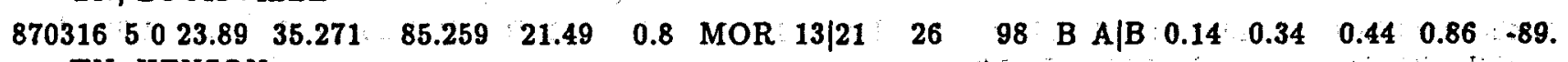
TN, HENSON

$\begin{array}{llllllllllllllllllllll}870324 & 2013 & 42.70 & 35.528 & 84.278 & 15.39 & 1.4 & \text { MOR } & 16 / 24 & 16 & 135 & \text { C B } & \text { B C } & 0.16 & 0.30 & 0.48 & 0.84 & 40 .\end{array}$ TN, MADISONVILLE

$\begin{array}{llllllllllllllllll}870327 & 729 & 30.46 & 35.556 & 84.229 & 18.52 & 4.2 & \text { MOR } 28 \mid 29 & 11 & 42 & \text { B } & \text { B } \mid A & 0.15 & 0.40 & 0.52 & 1.00 & 29 .\end{array}$ TN, VONORE

$\begin{array}{lllllllllllllllllllllll}870327 & 735 & 38.92 & 35.555 & 84.232 & 16.69 & 1.6 & \text { MOR } & 15 & 22 & 12 & 48 & \text { A A } & \text { A A } & 0.14 & 0.32 & 0.49 & 0.68 & -123\end{array}$ TN, VONORE; aftershock of 870327 07:29 event

$\begin{array}{lllllllllllllllll}870327 & 753 & 37.27 & 35.557 & 84.227 & 15.89 & -0.2 & \text { MOR } & 5 & 6 & 12 & 360 \text { : C B|D } & 0.11 & 0.91 & 1.53 & 1.87 & 28 .\end{array}$ .TN, VONORE; aftershock of 870327 07:29 event

$\begin{array}{llllllllllllllllll}870327 & 1623 & 4.96 & 35.546 & 84.230 & 14.95 & 1.5 & \text { MOR } & 22 \mid 33 & 2 & 85 & \text { B } & \text { B|A } & 0.20 & 0.34 & 0.58 & 0.65 & -129 .\end{array}$ TN, VONORE; aftershock of 870327 07:29 event

\begin{tabular}{ll|llllllllllllllllllllll}
870328 & 1530 & 16.62 & 35.657 & 84.390 & 8.90 & 0.6 & MOR & $11 \mid 14$ & 13 & 227 & C B & 0.19 & 0.55 & 0.87 & 2.13 & 34.
\end{tabular} TN, PHILADELPHIA; aftershock of 870327 07:29 event

$\begin{array}{lllllllllllllllllll}870329 & 8 & 4 & 51.23 & 35.558 & 84.220 & 15.80 & -1.2 & \text { MOR } & 4 & 7 & 2 & 225 & \text { D C|D } & 0.10 & 1.06 & 3.00 & 1.39 & 5\end{array}$

TN, VONORE; aftershock of 870327 07:29 event

$\begin{array}{lllllll}870401 & 817 & 21.09 & 35.546 & 84.228 & 15.34 & 1.0\end{array}$. MOR $19 \mid 25$

TN, VONORE; aftershock of 870327 07:29 event

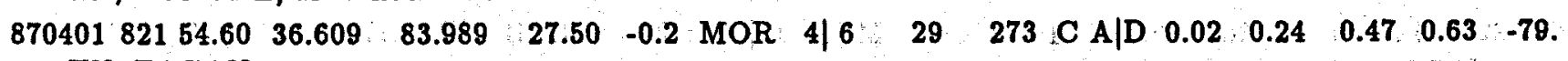
TN, EAGAN

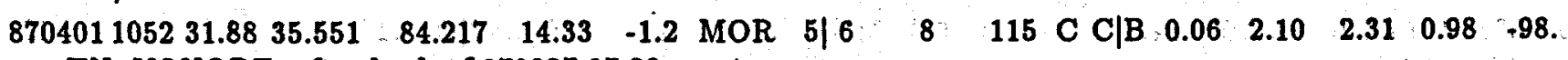
TN, VONORE; aftershock of 870327 07:29 event

$\begin{array}{lllllllllllllllllllll}870403 & 1212 & 20.18 & 35.590 & 84.440 & 15.00 & 0.2 & \text { MOR } & 3 & 6 & 7 & 276 & \text { D C } & 0.42 & 0.61 & 1.50 & 0.65 & 15 .\end{array}$ TN, SWEETWATER; aftershock of 870327 07:29 event

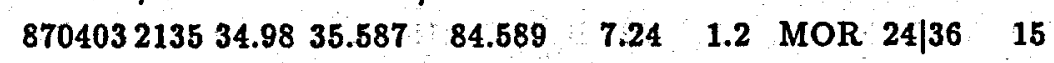
TN, NIOTA; aftershock of 870327 .07:29 event

$\begin{array}{lllllllllllllllllll}870404 & 031 & 24.03 & 35.471 & 84.337 & 9.62 & 0.3 & \text { MOR } & 9 \mid 18 & 3 & 122 & \text { B A } & 0.12 & 0.43 & 0.67 & 0.69 & 45\end{array}$ TN, MT VERNON; aftershock of $87032707: 29$ event

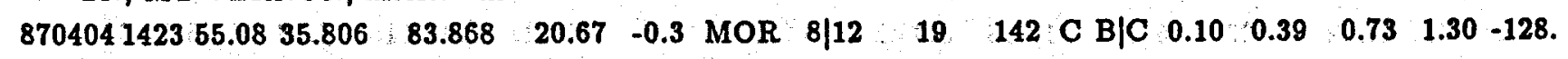
TN, WILDWOOD

$\begin{array}{lllllllllllllllllll}870405 & 7.95 .50 & 35.458 & 84.439 & 9.12 & -1.2 & \text { MOR } & 5 & 5 & 8 & 360 & D & \text { C } & 0.14 & 1.53 & 3.43 & 4.22 & 21 .\end{array}$ TN, ENGLEWOOD; aftershock of 870327 07:29 event

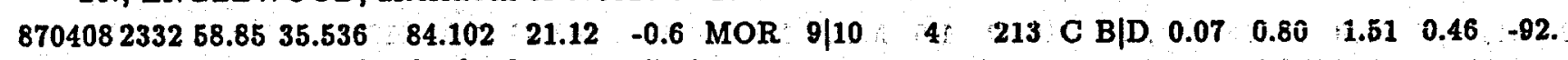
TN, YALLASSEE; aftershock of 870327 07:29 event

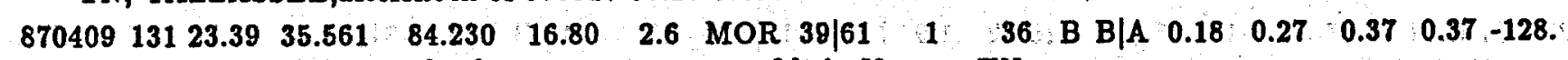
TN, VONORE; aftershock of 870327 07:29 event; felt in Vonore, TN area

$\begin{array}{llllllllllllllllll}870409 & 1367.63 & 35.575 & 84.243 & 16.43 & -1.2 & \text { MOR } & 7 \mid 7 & 5 & 127 & \text { B } & \text { B } & \text { B } & 0.02 & 0.36 & 0.71 & 0.25 & 2 .\end{array}$ TN, VONORE; aftershock of 870327 07:29 event 
DATE ORGIN LAT $^{\circ} \mathrm{N}$ LONG ${ }^{\circ} \mathrm{W}$ DEP MAG MOD ST|PH DMIN GAP Q SQD RMS ERH1 ERH2 ERZ AZM

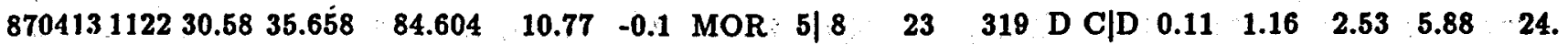
TN, PATTIE GAP; aftershock of 870327 07:29 event

$\begin{array}{llllllllllllllllllllll}870414.2228 & 32.05 & 34.678 & 85.092 & 11.60 & 1.6 & \text { MOR } & 8 \mid 13 & 39 & 146 & \text { C B } & \text { B } & 0.19 & 0.64 & 0.89 & 1.54 & 28 .\end{array}$ GA, VILLANOW

$\begin{array}{lllllllllllllllllll}870418 & 1843 & 25.25 & 35.323 & 84.542 & 13.95 & -0.2 & \text { MOR } & 9 \mid 12 & 8 & 235 & \text { C B } & \text { B D } & 0.16 & 0.70 & 1.24 & 1.19 & -110\end{array}$ TN, ETOWAH; aftershock of 870327 07:29 event

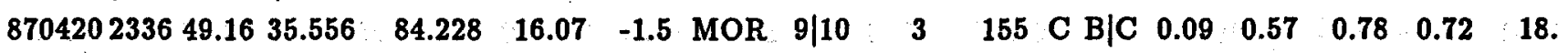
TN, VONORE; aftershock of 870327 07:29 event

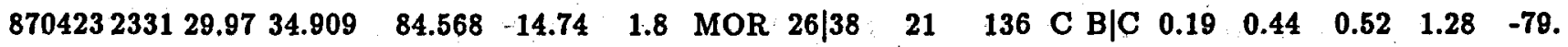
GA, HEMP TOP

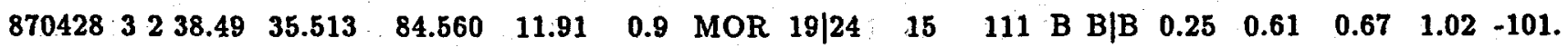
TN, NIOTA; aftershock of 870327 07:29 event

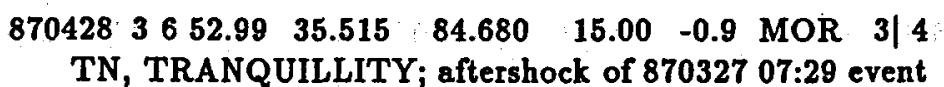
$\begin{array}{llllllllll}870428 & 339 & 38.22 & 35.521 & 84.552 & 11.98 & 2.0 & \text { MOR } & 23 \mid 36 & 16\end{array}$ TN, NIOTA; aftershock of 870327 07:29 event

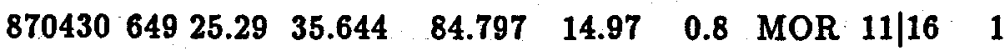
TN, SPRING CITY

8705052330.6136 .398 TN, LA FOLLETTE

$\begin{array}{lllllll}84.079 & 18.87 & 1.2 & \text { MOR } & 8 \mid 11 & 31\end{array}$ 0505194122.5035 .600 $\begin{array}{lllll}84.234 & 4.62 & 0.7 & \text { MOR } & 7 \mid 10\end{array}$ TN, VONORE

$870509 \quad 910 \quad 8.30 \quad 34.721$ GA, CATLETT

$870512121759.5735 .988: 83.998$ TN, KNOXVILLE

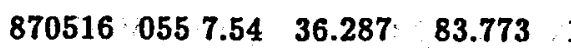
TN, MAYNARDVILLE

87052273622.3035 .036
TN, OOLTEWAH 85.041 $15.34 \quad 0.5$ MOR $11 \mid 17,24$ T25. 111744.2535 .520 TN, NIOTA

87052972420.9135 .708 TN, SPRING CITY

$87060125022.50 \quad 35.568$ TN, MADISONVILLE

870602161427.5535 .677 TN, WELCHLAND

$\begin{array}{llllllll}8706041719 & 41.02 & 36.623 & 85.269 & 18.18 & 3.0 & \text { MOR } 22 \mid 31 & 50\end{array}$ TN, DALE HOLLOW RESERVOIR SE

$\begin{array}{llllllllllllllllll}870609 & 2020 & 54.96 & 34.550 & 85.332 & 9.82 & 1.2 & \text { MOR } & 7 \mid 11 & 15 & 149 & \text { C } & \text { B } \mid \mathrm{C} & 0.17 & 0.84 & 1.47 & 1.18 & -29 .\end{array}$ GA, TRION

$\begin{array}{llllllllllllllll}870614 & 61729.80 & 35.286 & 84.235 & 16.69 & 1.5 & \text { MOR } 11 \mid 15 & 21 & 143 & \text { C B } & \text { BC } & 0.18 & 0.63 & 0.79 & 1.43 & -80\end{array}$ TN, BALD RIVER FALLS

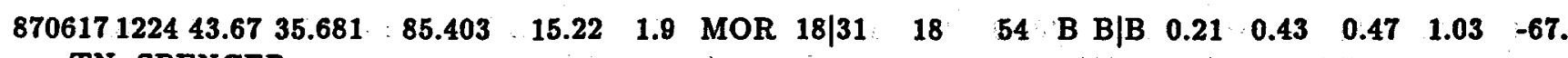
TN, SPENCER

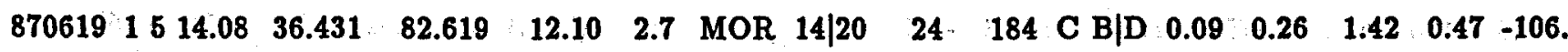
TN, SULLIVAN GARDENS 
DATE ORGIN LAT ${ }^{\circ}$ LONG $^{\circ} W$ DEP MAG MOD ST|PH DMIN GAP Q SQD RMS ERH1 ERH2 ERZ AZM $\begin{array}{llllllllllllllllllll}8707041047 & 24.96 & 35.540 & 84.445 & 16.14 & 0.9 & \text { MOR } & 15 \mid 23 & 24 & 121 & \text { B B|B } & 0.21 & 0.44 & 0.68 & 1.35 & -134 .\end{array}$ TN, SWEETWATER

$\begin{array}{llllllllllllllllllllllll}870707 & 15 & 3 & 33.72 & 35.560 & 84.182 & 14.33 & 0.7 & \text { MOR } & 7 \mid 12 & 12 & 214 & \text { C B } & 0.27 & 1.01 & 1.82 & 1.75 & 45 .\end{array}$ TN, VONORE

$\begin{array}{llllllllllllllllll}870709 & 1 & 3 & 54.07 & 34.782 & 82.954 & 5.00 & 1.9 & \text { MOR } & 15 \mid 22 & 17 & 224 & \text { D C } & 0.36 & 1.16 & 1.44 & 1.19 & -126 .\end{array}$ SC, OLD PICKENS; reservoir associated

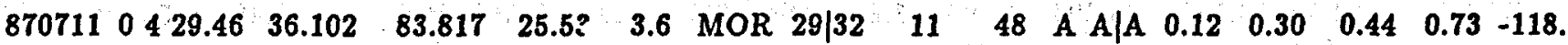
TN, JOHN SEVIER; felt in Knoxville, TN and surrounding counties

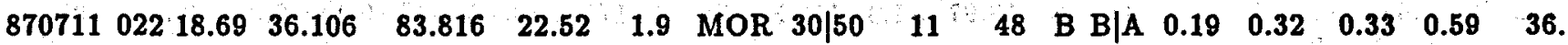
TN, JOHN SEVIER; aftershock of 870711 00:04 event

$\begin{array}{lllllllllllllllllllllll}870711 & 122 & 20.55 & 34.812 & 82.987 & 5.93 & 1.2 & \text { MOR } & 5 & 8 & 60 & 275 & D & \text { C } & 0.18 & 1.08 & 2.00 & 6.96 & -118 .\end{array}$ SC, OLD PICKENS; reservoir associated

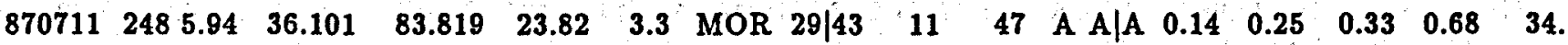
TN, JOHN SEVIER; felt in Knoxville, TN and surrounding counties; aftershock of 870711 00:04 event

$\begin{array}{llllllllllllllllllllllll}870711 & 321 & 53.50 & 34.794 & 82.999 & 2.98 & 1.2 & \text { MOR } & 5 & 8 & 62 & 222 & \text { D C|D } & 0.25 & 1.73 & 3.68 & 7.25 & -118 .\end{array}$ SC, OLD PICKENS; reservoir associated

$\begin{array}{lllllllllllllllll}8707112224 & 10.51 & 35.877 & 83.655 & 15.27 & 0.6 & \text { MOR } & 9 \mid 13 & 18 & 117 & \text { B } & \text { B } \mid \text { B } & 0.16 & 0.63 & 0.78 & 1.52 & 28 .\end{array}$ TN, BOYDS CREEK

87071423212.0035 .801 TN, MARYVILLE

$87071781911.15 \quad 35.986$ TN, SHOOKS GAP

870717153025.4036 .092 TN, JOHN SEVIER

$\begin{array}{llllllllllllllll}83.987 & 15.95 & 0.3 & \text { MOR } & 9 & 12 & 17 & 143 & \mathrm{C} & \mathrm{B} \mid \mathrm{C} & 0.18 & 0.69 & 0.84 & 1.87 & -87\end{array}$

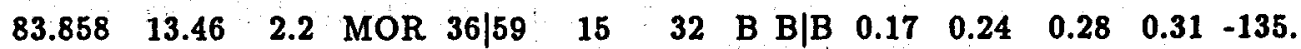

$\begin{array}{lllllllllllllll}83.811 & 22.17 & 0.5 & \text { MOR } & 9 & 13 & 9 & 123 & \text { B A } & \text { A B } & 0.11 & 0.47 & 0.74 & 0.67 & -80\end{array}$

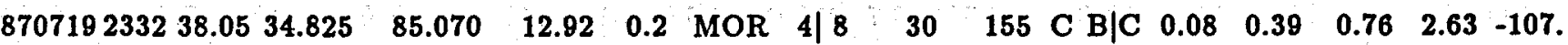
GA, TUNNEL HILL; Georgia/Tennessee state line

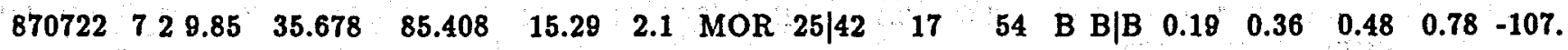
TN, SPENCER

$\begin{array}{llllllllllllllllllllll}870728 & 2357 & 59.61 & 35.251 & 84.126 & 6.51 & -0.2 & \text { MOR } & 7 / 10 & 31 & 155 & \mathrm{C} & \mathrm{C} / \mathrm{C} & 0.23 & 0.95 & 1.13 & 38.88 & 44 .\end{array}$ TN, BALD RIVER FALLS

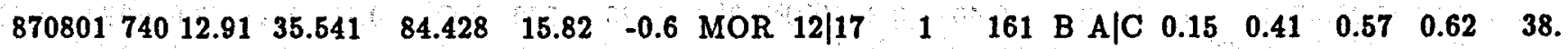
TN, SWEETWATER

$870808 \quad 04147.78$ TN, FELKER

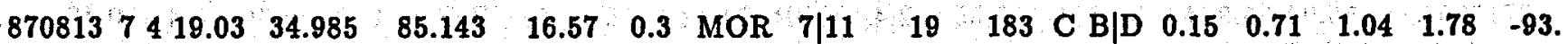
GA, EAST RIDGE

$8708131337 \quad 0.82 \quad 35.625$ TN, LOUDON

87081422126.9635 .439
TN, RICEVILLE

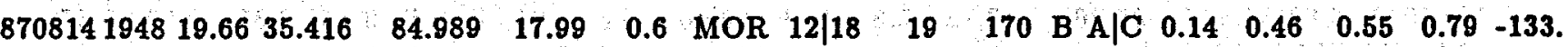
TN, BIG SPRING

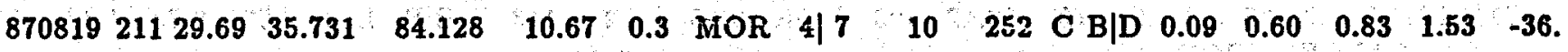
TN, MEADOW

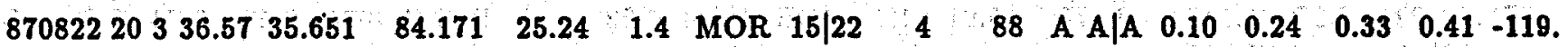
TN, MEADOW

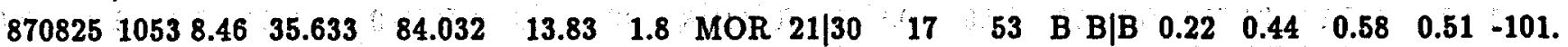
TN, BINFIELD 
DATE ORGIN LAT ${ }^{\circ}$ LONG $^{\circ} W$ DEP MAG MOD ST|PH DMIN GAP Q SQD RMS ERH1 ERH2 ERZ AZM

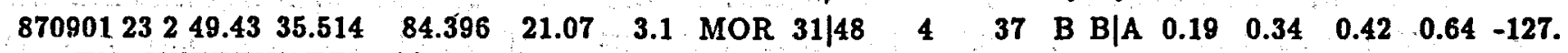
TN, SWEETWATER; felt in Athens, TN area

$\begin{array}{llllllllllllllllll}8709012315 & 42.44 & 35.514 & 84.397 & 19.19 & 1.2 & \text { MOR } & 17 \mid 27 & 22 & 64 & \text { B } & \text { B|B } & 0.18 & 0.37 & 0.48 & 0.83 & 39 .\end{array}$ TN, SWEETWATER

$\begin{array}{lllllllllllllllll}8709012328 & 26.66 & 35.511 & 84.409 & 16.40 & -0.2 & \text { MOR } & 5 & 6 & 25 & 360 & \text { D C C D } & 0.10 & 0.82 & 1.43 & 3.00 & 25 .\end{array}$ TN, SWEETWATER

$\begin{array}{lllllllllllllllllllllllllll}870902 & 757 & 21.16 & 34.748 & 85.203 & 11.96 & 1.8 & \text { MOR } & 22 \mid 38 & 18 & 109 & \text { B } & \text { B|B } & 0.25 & 0.48 & 0.56 & 0.69 & 37 .\end{array}$ GA, CATLETT

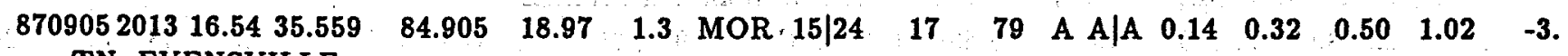
TN, EVENSVILLE

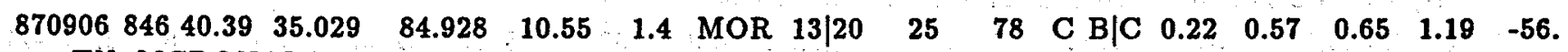
TN, MCDONALD

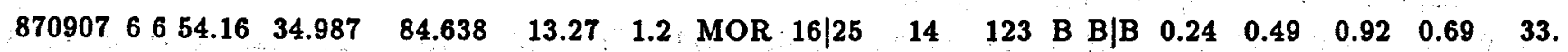
GA, TENNGA; Georgia/Tennessee state line

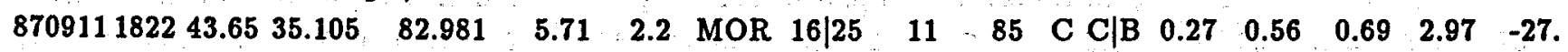
NC, REID

$\begin{array}{llllllllllllllllll}8709162132 & 32.34 & 36.078 & 83.696 & 17.62 & 1.3 & \text { MOR } & 5 & \mid 8 & 19 & 173 & \text { C C } & \text { C C } & 0.20 & 1.50 & 1.78 & 3.61 & -88 .\end{array}$ TN, MASCOT

$\begin{array}{lllllllllllllllllll}870919 & 322 & 50.07 & 35.534 & 84.698 & 17.29 & 1.2 & \text { MOR } & 13 \mid 20 & 3 & 104 & \text { B } & \text { B|B } & 0.19 & 0.53 & 0.58 & 0.68 & -81 .\end{array}$ TN, TRANQUILLITY

$\begin{array}{lllllllllllllllll}8709192156 & 44.16 & 35.853 & 82.049 & 6.24 & 2.0 & \text { MOR } & 18 \mid 31 & 25 & 63 & \text { C } & \text { C } \mid C & 0.33 & 0.62 & 0.72 & 2.40 & -124 .\end{array}$ NC, LITTLE SWITZERLAND

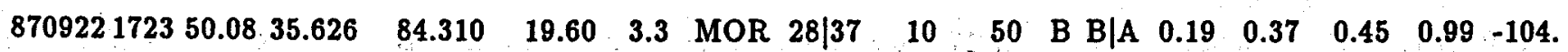
TN, LOUDON; felt in Vonore, Philadelphia, Binfield and Madisonville, TN

$\begin{array}{lllllllllllllllllll}871002 & 211 & 36.30 & 35.145 & 84.736 & 8.82 & 0.7 & \text { MOR } & 4 & 6 & 31 & 243 & \text { D } & \text { D } & 0.31 & 2.72 & 7.43 & 12.78 & 21 .\end{array}$ TN, BENTON

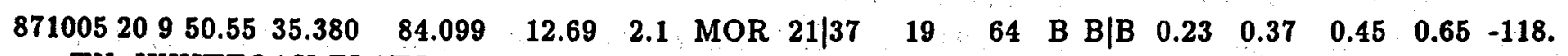
TN, WHITEOAK FLATS

$\begin{array}{lllllllllllllllll}8710101139 & 11.49 & 35.708 & 84.554 & 19.27 & 0.7 & \text { MOR } & 7 \mid 11 & 26 & 237 & \text { C A|D } & 0.05 & 0.21 & 0.51 & 0.55 & -117 .\end{array}$ TN, PATTIE GAP

$\begin{array}{lllllllllllllllllllllll}871012 & 851 & 45.53 & 35.846 & 84.023 & 0.46 & -0.8 & \text { MOR } & 3 / 4 & 26 & 360 & \text { D C D } & 0.15 & 1.25 & 3.04 & 99.00 & -71\end{array}$ TN, LOUISVILLE

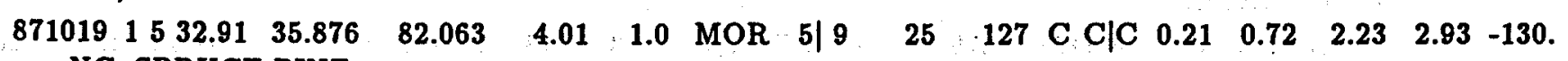
NC, SPRUCE PINE

871020224955.9435 .844 TN, CAVE CREEK

$8710239407.19 \quad 35.830$ TN, CONCORD

871023105251.6734 .889 GA, HEMP TOP

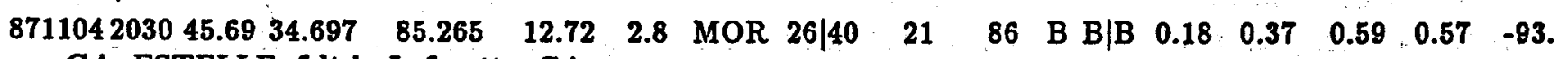
GA, ESTELLE; felt in Lafayette, GA area

871104221739.2435 .539 TN, CALDERWOOD

$87110422198.27 \quad 35.542$ TN, CALDERWOOD

$\begin{array}{llllllllllllllllllllllll}871108 & 515 & 7.16 & 34.760 & 85.369 & 9.50 & 0.5 & \text { MOR } & 11 \mid 18 & 18 & 103 & \text { B } & \text { B|B } & 0.23 & 0.54 & 0.91 & 1.14 & 6 .\end{array}$ GA, KENSINGTON

$\begin{array}{llllllllllllllll}83.972 & 16.42 & \text { U.6 } & \text { MOR } & 9 / 15 & 22 & 113 & \text { B } & \text { B } \mid B & 0.20 & 0.59 & 0.78 & 1.83 & -82 .\end{array}$

$\begin{array}{llllllllllllllll}83.970 & 15.91 & 1.5 & \text { MOR } & 15 & 24 & 22 & 90 & \text { B } & \text { B } \mid \text { B } & 0.24 & 0.53 & 0.73 & 1.83 & -68\end{array}$ 
DATE ORGIN LAT $^{\circ} N$ LONG ${ }^{\circ} W$ DEP MAG MOD ST|PH DMIN GAP Q SQD RMS ERH1 ERH2 ERZ AZM $\begin{array}{lllllllllllllllllllllll}87108 & 846 & 51.15 & 36.301 & 83.479 & 10.84 & 1.5 & \text { MOR } & 9 / 13 & 28 & 180 & \text { C B|D } & 0.26 & 0.84 & 1.41 & 1.85 & -106\end{array}$ TN, AVONDALE

$\begin{array}{lllllllllllllllllll}871110 & 838 & 22.92 & 35.465 & 85.140 & 11.00 & 1.7 & \text { MOR } & 18 & 18 & 42 & 56 & \text { C B } & \text { B } & 0.28 & 0.86 & 0.99 & 1.61 & -85 .\end{array}$ TN, BRAYTON

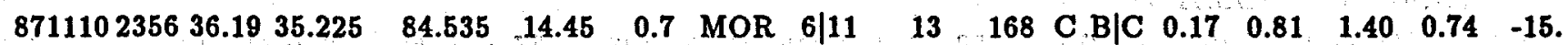
TN, OSWALD DOME

$\begin{array}{lllllllllllllllllllll}871111 & 72 & 40.29 & 35.699 & 84.144 & 19.07 & 1.8 & \text { MOR } 22 / 41 & 7 & 43 & \text { B } & \text { B|A } & 0.21 & 0.35 & 0.47 & 0.64 & -123 .\end{array}$ TN, MEADOW

$\begin{array}{llllllllllllllllllllllll}871115 & 643 & 51.08 & 35.737 & 84.297 & 7.43 & -0.1 & \text { MOR } & 4 \mid 7 & 11 & 160 & \text { C } & \text { B } / C & 0.07 & 0.37 & 0.63 & 1.12 & -14 .\end{array}$ TN, LOUDON

$\begin{array}{llllllllllllllllllllll}871118 & 559 & 11.28 & 35.662 & 83.616 & 5.57 & 0.0 & \text { MOR } & 3 \mid 5 & 14 & 232 & \text { D } & \text { C|D } & 0.13 & 1.95 & 15.19 & 16.32 & 17 .\end{array}$ TN, GATLINBURG

$\begin{array}{lllllllllllllllllll}871121 & 1415 & 7.09 & 35.568 & 84.176 & 12.37 & -0.1 & \text { MOR } & 3 \mid 5 & 11 & 240 & \text { D C|D } & 0.12 & 1.65 & 5.85 & 7.26 & -120 .\end{array}$ TN, VONORE

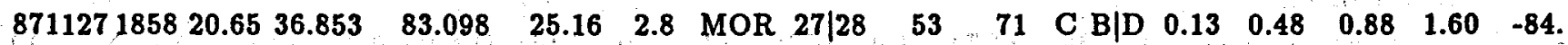
KY, PENNINGTON GAP; felt in Harlan, $\mathrm{KY}$ and Pennington Gap, VA area

$\begin{array}{lllllllllllllllllll}871127 & 1858 & 29.34 & 36.852 & 83.110 & 26.78 & 3.5 & \text { MOR } & 26 \mid 35 & 57 & 71 & \text { C B B D } & 0.14 & 0.29 & 0.66 & 1.87 & -89 .\end{array}$ KY, PENNINGTON GAP; felt in Harlan, $\mathrm{KY}$ and Pennington Gap, VA area;

$\begin{array}{lllllllllllllllllllllll}871128 & 1525 & 1.52 & 35.552 & 84.227 & 11.19 & 0.0 & \text { MOR } & 3 \mid 6 & 13 & 191 & \text { D D } & 0.12 & 1.61 & 8.07 & 5.75 & 30 .\end{array}$ TN, VONORE

$\begin{array}{llllllllllllllllll}871129 & 210 & 51.55 & 36.854 & 83.104 & 9.29 & 1.8 & \text { MOR } & 6 \mid 10 & 53 & 226 & \text { C B|D } & 0.12 & 0.74 & 1.49 & 1.17 & -102 .\end{array}$ KY, PENNINGTON GAP

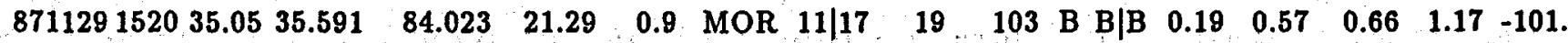
TN, TALLASSEE

$\begin{array}{llllllllllllllllllll}871130 & 72 & 44.14 & 36.096 & 83.802 & 20.60 & 0.7 & \text { MOR } & 7 \mid 10 & 12 & 194 & \text { C B|D } & 0.19 & 1.07 & 1.30 & 1.76 & -85\end{array}$ TN, JOHN SEVIER

$\begin{array}{lllllllllllllllll}871201 & 814 & 59.75 & 35.397 & 84.290 & 13.61 & 0.4 & \text { MOR } & 10 \mid 13 & 17 & 184 & \mathrm{C} \text { A|D } & 0.14 & 0.45 & 0.83 & 0.71 & 40 .\end{array}$ TN, MT VERNON

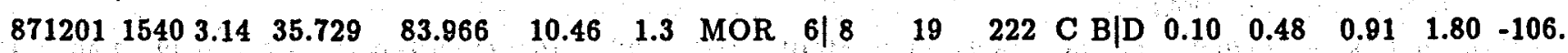
TN, BLOCKHOUSE

$\begin{array}{lllllllllllllllllllllllll}871203 & 422 & 36.13 & 35.830 & 84.683 & 24.24 & -1.1 & \text { MOR } & 5 & 8 & 24 & 263 & \text { D } & \text { C|D } & 0.15 & 1.01 & 1.39 & 2.98 & -97 .\end{array}$ TN, ROCKWOOD

$\begin{array}{llllllllllllllllllllllll}871206 & 1453 & 5.77 & 34.761 & 85.271 & 8.66 & 2.9 & \text { MOR } & 31 \mid 55 & 24 & 58 & \text { C B/C } & 0.22 & 0.32 & 0.39 & 0.60 & -110 .\end{array}$ GA, KENSINGTON; felt in Lafayette,GA area

$\begin{array}{llllllllllllllllllllllll}871207 & 44 & 48.31 & 36.014 & 84.433 & 22.47 & 1.9 & \text { MOR } & 19 \mid 36 & 16 & 75 & \text { A A } & 0.14 & 0.30 & 0.32 & 0.57 & -106 .\end{array}$ TN, PETROS

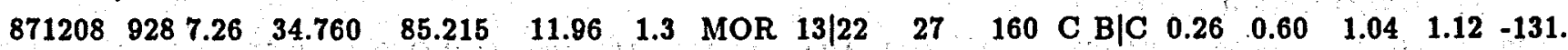
GA, NICKAJACK GAP

$\begin{array}{llllllllllllllllllllll}871211 & 415 & 10.93 & 35.234 & 84.248 & 8.32 & -0.1 & \text { MOR } & 3 \mid 5 & 21 & 280 & \text { D D } & \text { D } & 0.24 & 2.12 & 17.41 & 49.30 & 5 .\end{array}$ NC, UNAKA

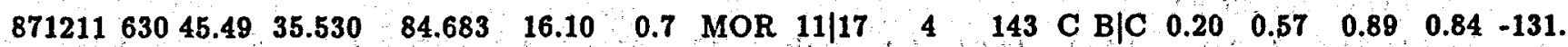
TN, TRANQUILLITY

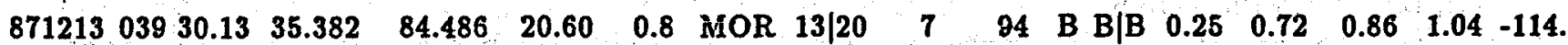
TN, ENGLEWOOD

$\begin{array}{llllllllllllllllllllllllll}871214 & 713 & 20.13 & 35.762 & 84.208 & 19.52 & 1.6 & \text { MOR } & 21 \mid 35 & 11 & 49 & \text { B } & \text { B|A } & 0.17 & 0.33 & 0.38 & 0.55 & -132 .\end{array}$ TN, CONCORD

$\begin{array}{llllllllllllllllllllll}871215 & 1020 & 21.08 & 35.775 & 84.381 & 19.02 & 0.4 & \text { MOR } & 7 \mid 12 & 16 & 157 & \text { B A } & \text { AlC } & 0.08 & 0.37 & 0.47 & 0.53 & 40 .\end{array}$ TN, CAVE CREEK 
DATE ORGIN LAT ${ }^{\circ}$ LONG $^{\circ} W$ DEP MAG MOD ST|PH DMIN GAP Q SQD RMS ERH1 ERH2 ERZ AZM $\begin{array}{lllllllllllllllllllllll}871216 & 1122 & 41.98 & 35.644 & 84.142 & 16.01 & 0.5 & \text { MOR } & 6 \mid 10 & 7 & 155 & \text { C B } & 0.16 & 0.70 & 2.66 & 1.95 & -113 .\end{array}$ TN, MEADOW

$\begin{array}{lllllllllllllllllllllllll}871218 & 8 & 8 & 59.36 & 36.151 & 86.100 & 15.34 & 1.3 & \text { MOR } & 15 \mid 24 & 29 & 67 & \text { B B|B } & 0.18 & 0.48 & 0.72 & 2.51 & -20 .\end{array}$ TN, NEW MIDDLETON

$871218232017.4135 .104 \quad 82.970$ NC, REID

$871223 \quad 6429.41 \quad 35.281 \quad 84.219$ TN, BALD RIVER FALLS

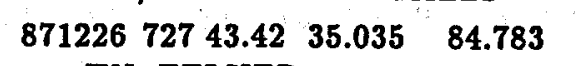
TN, FELKER

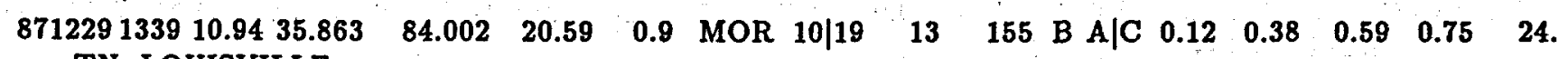
TN, LOUISVILLE

$88010232450.97 \quad 35.007 \quad 84.356$ TN, ISABELLA

$8801021743 \quad 58.3935 .590 \quad 84.268$ TN, MADISONVILLE

$880103224721.4035 .046 \quad 84.506$ TN, CANEY CREEK

$88010455730.22 \quad 35.004 \quad 84.345$ TN, ISABELLA

$880105123214.6735 .010 \quad 84.373$

TN, ISABELLA

$8801051338 \quad 50.7435 .021 \quad 84.393$ TN, DUCKTOWN

$880108232751.5235 .282 \quad 84.183$
TN, BALD RIVER FALLS $6.44-0.3$ MOR $3 \mid 5 \quad 3$

$\begin{array}{llllllllllllllll}11.74 & -1.0 & \text { MOR } & 3 \mid 5 & 10 & 181 & \text { D C C D } & 0.03 & 1.86 & 7.50 & 6.54 & -123\end{array}$

$\begin{array}{llllllllllllll}11.74 & 0.3 & \text { MOR } & 3 \mid 4 & 25 & 208 & \text { D } & \text { C } & \text { D } & 0.15 & 1.95 & 3.50 & 18.10 & 25\end{array}$

$\begin{array}{llllllllllllll}7.77 & 0.6 & \text { MOR } & 3 / 4 & 34 & 360 & \text { D C|D } & 0.12 & 2.27 & 3.23 & 48.47 & -22 .\end{array}$

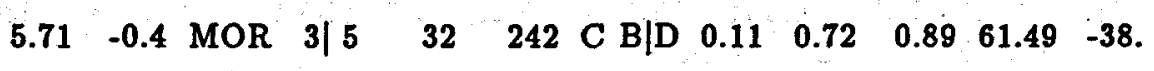

$\begin{array}{lllllllllllllll}10.28 & -0.2 & \text { MOR } & 3 / 4 & 31 & 360 & \text { D C } & 0.00 & 2.25 & 3.76 & 24.13 & -3\end{array}$

$\begin{array}{llllllllllllll}11.40 & 1.2 & \text { MOR } & 12 \mid 16 & 25 & 299 & \mathrm{C} & \mathrm{B} \mid \mathrm{D} & 0.24 & 0.73 & 1.01 & 1.24 & -134\end{array}$

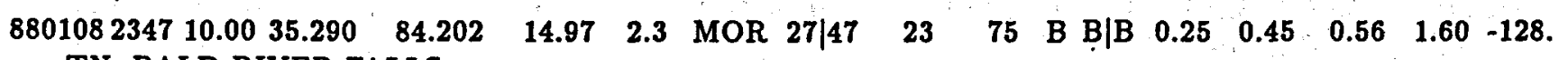
TN, BALD RIVER FALLS

880109 O $328.66 \quad 35.282 \quad 84.186$ TN, BALD RIVER FALLS

$88010901655.41 \quad 35.286 \quad 84.194$ TN, BALD RIVER FALLS

$\begin{array}{llll}880109 & 024 & 36.83 \quad 35.281 & 84.183\end{array}$ TN, BALD RIVER FALLS

$\begin{array}{lllll}880109 & 17 & 40.62 \quad 35.276 & 84.200\end{array}$ TN, BALD RIVER FALLS

$\begin{array}{lllll}880109 & 115 & 5.25 & 35.296 & 84.198\end{array}$ TN, BALD RIVER FALLS

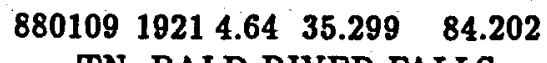
TN, BALD RIVER FALLS

$880109221124.45 \quad 35.285 \quad 84.180$ TN, BALD RIVER FALLS

$\begin{array}{lllll}880110 & 410 & 3.59 & 35.024 & 84.381\end{array}$ TN, DUCKTOWN

$\begin{array}{lllll}880110 & 432 & 3.51 & 35.005 & 84.386\end{array}$ TN, DUCKTOWN

$880110132258.08 \quad 35.285 \quad 84.178$

$\begin{array}{llllllllllll}15.22 & 1.1 & \text { MOR } & 11 \mid 17 & 25 & 101 & \text { C C C B } & 0.23 & 0.75 & 0.82 & 2.69 & 29\end{array}$

$\begin{array}{lllllllllllll}12.64 & 2.9 & \text { MOR } & 28 \mid 51 & 24 & 68 & \text { B } & \text { B|B } & 0.27 & 0.42 & 0.53 & 0.58 & -128 .\end{array}$

$\begin{array}{llllllllllllllll}12.09 & 1.8 & \text { MOR } & 13 \mid 21 & 25 & 102 & \text { C } & \text { B } \mid C & 0.27 & 0.64 & 0.80 & 1.13 & -110\end{array}$

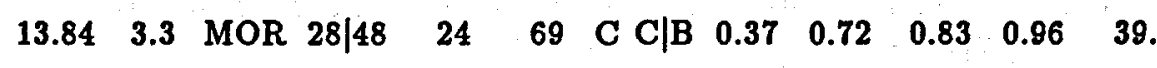

$\begin{array}{lllllllllllll}7.82 & 1.8 & \text { MOR } & 14 \mid 20 & 24 & 99 & \mathrm{C} & \mathrm{B} \mid \mathrm{C} & 0.25 & 0.61 & 0.72 & 1.77 & -102 .\end{array}$

$\begin{array}{lllllllllllll}14.85 & 1.0 & \text { MOR } & 6 \mid 9 & 23 & 299 & \text { D C|D } & 0.17 & 0.82 & 1.87 & 2.97 & -134\end{array}$

$\begin{array}{llllllllllll}13.64 & 1.4 & \text { MOR } & 13 \mid 18 & 25 & 223 & \text { D C|D } & 0.40 & 1.10 & 1.30 & 1.34 & 35 .\end{array}$

$\begin{array}{lllllllllllllll}7.88 & 0.1 & \text { MOR } & 3 & 5 & 32 & 247 & \text { D C C D } & 0.18 & 1.74 & 3.11 & 36.96 & 17 .\end{array}$

$\begin{array}{llllllllllllll}8.69 & -0.8 & \text { MOR } & 3 \mid & 5 & 30 & 249 & \text { D C C D } & 0.02 & 1.96 & 3.22 & 29.43 & 3 .\end{array}$

$\begin{array}{llllllllllllll}6.03 & 0.2 & \text { MOR } & 5 & 7 & 26 & 300 & \text { D C C D } & 0.14 & 1.05 & 1.75 & 77.02 & 32 .\end{array}$ TN, BALD RIVER FALLS 
DATE ORGIN LAT ${ }^{\circ} \mathrm{N}$ LONG ${ }^{\circ} \mathrm{W}$ DEP MAG MOD ST|PH DMIN GAP Q SQD RMS ERH1 ERH2 ERZ AZM

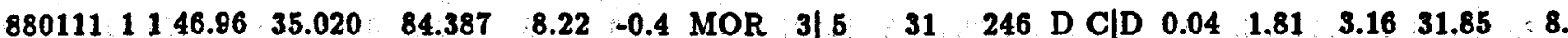
TN, DUCKTOWN

880111122159.0735 .467 TN, MT VERNON

880113235330.6835 .022 TN, DUCKTOWN

$88011323545.87 \quad 35.010$ TN, DUCKTOWN

$880114 \quad 2477.67 \quad 35.005$ TN, DUCKTOWN

880114102657.8335 .021 TN, DUCKTOWN

880114214711.1335 .013 TN, DUCKTOWN

$88011512514.80 \quad 35.009$ TN, ISABELLA

$88011513531.28 \quad 35.011$ TN, ISABELLA

$88011523830.92 \quad 35.009$ TN, DUCKTOWN

$88011619133.13 \quad 35.002$ TN, DUCKTOWN

$880118 \quad 54752.00 \quad 35.160$ TN, BENTON

$88011821535.03 \quad 35.539$ TN, VONORE

880124213611.9935 .284 TN, TELLICO PLAINS

$88020484930.00 \quad 35.007$ TN, DUCKTOWN

$880207 \quad 83929.25 \quad 36.331$ TN, DUTCH VALLEY

880211221311.8735 .468 TN, MT VERNON

8802121447 -38.63 35.732 TN, BLOCKHOUSE

$880213 \quad 5531.40 \quad 35.354$ TN, CALHOUN 88021431325.8834 .997 GA, EPWORTH 802144452.2535 .592
TN, MADISONVILLE 880214114046.9436 .085 TN, NEW MARKET 880216152654.7036 .617 TN, BLOUNTVILLE

$\begin{array}{llllllllllllllll}84.259 & 19.00 & 1.0 & \text { MOR } & 11 \mid 18 & 17 & 125 & \text { C C C B } & 0.33 & 0.89 & 1.70 & 2.21 & -120 .\end{array}$ $\begin{array}{lllllllllllllll}84.383 & 6.04 & -0.8 & \text { MOR } & 3 \mid & 5 & 32 & 247 & \text { D C C D } & 0.12 & 2.02 & 3.08 & 99.00 & 10 .\end{array}$ $\begin{array}{llllllllllllllll}84.391 & 12.27 & -0.5 & \text { MOR } & 3 \mid & 5 & 30 & 247 & D & C \mid D & 0.13 & 2.03 & 3.20 & 15.64 & 6\end{array}$ $\begin{array}{lllllllllllllll}84.379 & 7.65 & 0.5 & \text { MOR } & 3 \mid 6 & 31 & 134 & \text { C } & \text { C } \mid C & 0.20 & 0.60 & 0.86 & 12.58 & -19 .\end{array}$ $\begin{array}{llllllllllllllll}84.398 & 8.80 & -0.3 & \text { MOR } & 3 \mid 5 & 30 & 243 & \text { D } & \text { C } \mid D & 0.11 & 1.83 & 2.57 & 26.98 & -15\end{array}$

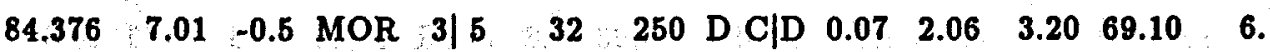
$\begin{array}{llllllllllllllll}84.366 & 10.50 & -0.4 & \text { MOR } & 3 \mid & 5 & 32 & 253 & D & C \mid D & 0.05 & 1.93 & 2.91 & 20.06 & 9\end{array}$ $\begin{array}{lllllllllllllllll}84.363 & 6.77 & -0.4 & \text { MOR } & 3 \mid & 5 & 33 & 253 & \text { D } & \text { C } & \text { D } & 0.14 & 2.48 & 3.15 & 97.68 & -8 .\end{array}$ $\begin{array}{lllllllllllllll}84.394 & 19.25 & -0.8 & \text { MOR } & 3 & 5 & 30 & 246 & \mathrm{D} & \mathrm{C} \mid \mathrm{D} & 0.07 & 2.85 & 4.15 & 15.74 & 4 .\end{array}$ $\begin{array}{lllllllllllllllll}84.378 & 6.00 & 0.1 & \text { MOR } & 3 \mid & 6 & 31 & 135 & \text { C } & \text { C } \mid C & 0.08 & 0.29 & 0.43 & 25.51 & -3\end{array}$ \begin{tabular}{lllll|lllllllllll}
84.694 & 9.20 & 1.4 & MOR & 3 & 5 & 28 & 234 & D & D & D & 0.06 & 1.60 & 10.43 & 5.63 & -74.
\end{tabular} \begin{tabular}{lllll|llllllllllll}
84.140 & 6.25 & 0.2 & MOR & 4 & 6 & 15 & 255 & D & C $\mid D$ & 0.13 & 0.20 & 1.61 & 7.06 & -120
\end{tabular} \begin{tabular}{lllll|llllllllll}
84.278 & 5.10 & -0.2 & MOR & 4 & 5 & 17 & 344 & D & D|D & 0.24 & 2.43 & 10.46 & 6.14 & 23.
\end{tabular} $\begin{array}{lllllllllllllllllll}84.375 & 5.66 & -0.1 & \text { MOR } & 3 & 5 & 31 & 251 & \mathrm{D} & \mathrm{C} \mid \mathrm{D} & 0.07 & 1.71 & 1.87 & 9.20 & -115\end{array}$ $\begin{array}{lllllllllllllll}83.512 & 14.84 & 1.2 & \text { MOR } & 8 & 12 & 30 & 195 & D & C & 0.21 & 0.54 & 1.65 & 6.11 & -106 .\end{array}$ $\begin{array}{lllllllllllllll}84.311 & 20.27 & 2.3 & \text { MOR } & 29 \mid 53 & 13 & 50 & \text { B } & \text { B|A } & 0.19 & 0.27 & 0.40 & 0.64 & 42 .\end{array}$ $\begin{array}{lllllllllllll}83.991 & 16.98 & 2.1 & \text { MOR } 21 \mid 36 & 21 & 56 & \text { B } & B \mid B & 0.21 & 0.39 & 0.50 & 1.05 & -113\end{array}$ $\begin{array}{lllllllllllllll}84.667 & 12.44 & 2.5 & \text { MOR } & 28 \mid 50 & 1 & 33 & \text { B } & \text { B|A } & 0.19 & 0.28 & 0.33 & 0.34 & 38 .\end{array}$ $\begin{array}{llllllllllllllll}84.411 & 13.16 & -0.6 & \text { MOR } & 3 \mid 4 & 28 & 360 & \text { D C C D } & 0.14 & 2.28 & 3.84 & 16.14 & -9 .\end{array}$ $\begin{array}{lllllllllllllll}84.329 & 12.58 & 1.6 & \text { MOR } & 23 / 31 & 10 & 68 & \text { B } & \text { B|A } & 0.16 & 0.27 & 0.40 & 0.37 & -124\end{array}$ $\begin{array}{lllllllllllllllllllll}83.598 & 12.06 & 1,9 & \text { MOR } & 9 / 14 & 25 & 155 & \mathrm{C} & \mathrm{B} / \mathrm{C} & 0.20 & 0.66 & 0.81 & 1.20 & -33\end{array}$ $\begin{array}{llllllllllllllll}82.288 & 10.86 & 3.2 & \text { MOR } & 16 \mid 24 & 24 & 180 & D & C & 0.32 & 0.80 & 2.02 & 1.29 & -96\end{array}$ $\begin{array}{llllllllllllllllll}8802171130 & 24.07 & 36.265 & 83.455 & 1.93 & 0.7 & \text { MOR } & 3 / 5 & 28 & 219 & D & \text { C|D } & 0.07 & 2.45 & 18.19 & 99.00 & -107\end{array}$ TN, AVONDALE 
DATE ORGIN LAT ${ }^{\circ}$ LONG ${ }^{\circ}$ W DEP MAG MOD ST|PH DMIN GAP Q SQD RMS ERH1 ERH2 ERZ AZM $\begin{array}{lllllllllllllllllllll}880218 & 037 & 45.50 & 35.340 & 83.836 & 6.05 & 3.5 & \text { MOR } & 36 / 52 & 36 & 48 & \text { C B B } & 0.28 & 0.42 & 0.55 & 1.50 & 45\end{array}$ NC, ROBBINSVILLE

$\begin{array}{llllllllllllllllllll}880220 & 2031 & 2.39 & 35.862 & 83.604 & 21.27 & 1.9 & \text { MOR } & 19 / 32 & 18 & 63 & \text { B } & \text { B|A } & 0.15 & 0.29 & 0.39 & 0.54 & -110\end{array}$ TN, PIGEON FORGE 88030251829.3435 .223 NC, UNAKA 880302182810.5536 .299 TN, AVONDALE $8803046559.86 \quad 35.000$
TN, DUCKTOWN $8803067628.63 \quad 34.970$ GA, EAST RIDGE

880311163932.6835 .811 TN, MARYVILLE

88033124529.6335 .200 NC, UNAKA

$880402 \quad 010 \quad 10.88 \quad 35.577$ TN, TALLASSEE

880402171835.1235 .536 TN, TALLASSEE

88041454235.5435 .50483 .419
NC, CLINGMANS DOME

$8804231116.64 \quad 35.781 \quad 84.208$ TN, CONCORD

880424 0 $847.61 \cdot 36.112 \quad 83.744$ TN, MASCOT
$8804260180.06 \quad 35.02$
TN, HUNTLAND

$880501 \quad 034 \quad 52.23 \quad 35.145$

TN, BENTON

880502111332.0336 .105 . TN, MASCOT

$880505 \quad 81926.60 \quad 34.933$ GA, RINGGOLD

$880508 \quad 623 \quad 4.29 \quad 35.029$ TN, ISABELLA

$\begin{array}{lll}880508 \quad 756 \quad 36.62 & 35.169 & 84.884\end{array}$ TN, SOUTH CLEVELAND

$\begin{array}{lllll}880508 \quad 852 & 51.08 & 34.991 & 84.388\end{array}$ GA, EPWORTH

$88050810624.93 \quad 35.012 \quad 84.385$ TN, DUCKTOWN

$880515 \cdot 9043.87 \times 34.533$

$\begin{array}{lllllllllllllll}85.521 & 3.84 & 0.9 & \text { MOR } & 3 & 5 & 131 & 356 & \text { D D D } & 0.05 & 25.67 & 51.47 & 89.00 & -29\end{array}$ AL, VALLEY HEAD

880519112157.0635 .811

$\begin{array}{llllllllllllllllll}84.138 & 13.85 & 0.9 & \text { MOR } & 4 & 7 & 17 & 269 & \text { D C } & 0 \text { D } & 0.10 & 0.94 & 2.69 & 0.67 & -110\end{array}$ TN, CONCORD

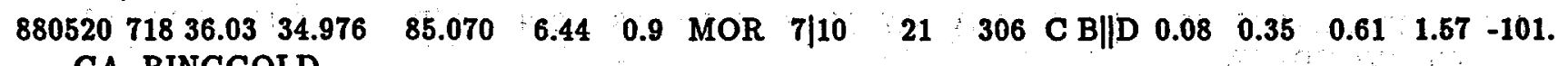
GA, RINGGOLD 
DATE ORGIN LAT ${ }^{\circ}$ LONG ${ }^{\circ}$ W DEP MAG MOD ST|PH DMIN GAP Q SQD RMS ERH1 ERH2 ERZ AZM

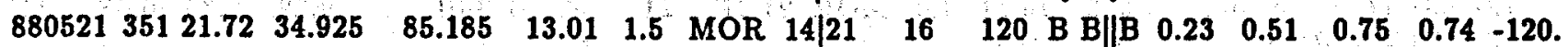
GA, EAST RIDGE

$\begin{array}{llllllllllllllllllllllllll}880522 & 15 & 0 & 31.79 & 36.967 & 83.775 & 9.29 & 2.2 & \text { MOR } & 17 \mid 30 & 80 & 207 & \text { C B } & 0.25 & 0.67 & 1.50 & 1.24 & -98 .\end{array}$ KY, FOUNT

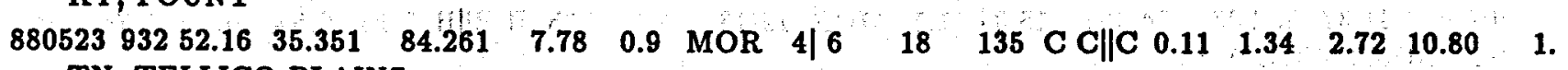
TN, TELLICO PLAINS

880526.95340 .5235 .894
TN, KNOXVILLE

88052715625.8535 .711 TN, BLOCKHOUSE

880603154753.1235 .260 TN, HENSON GAP

$88060672257.60 \quad 36.034$ TN, MASCOT

880610133126.4335 .621 TN, NIOTA

880611124528.3235 .298 TN, MECCA

$88061415459.20 \quad 35.421$ TN, ENGLEWOOD

880614121040.3835 .356 TN, CALHOUN

880614133751.7036 .146 TN, LUTTRELL

880615144616.5534 .630 SC, ANDERSON

880618103628.1435 .569 TN, VONORE

$88062075018.39 \quad 35.835$ TN, CONCORD

$880622 \quad 1648 \quad 2.98 \quad 35.659$ TN, MEADOW

880630125731.5036 .137

TN, TALBOTT

$88070275848.47 \quad 35.019$ TN, DUCKTOWN

$8807031128 \quad 8.30 \quad 35.687$ TN, LOUDON

880704225618.7936 .856 KY, BARBOURVILLE

$880706 \quad 15848.21 \quad 36.315 \quad 83.525$ TN, DUTCH VALLEY

$88071293931.24 \quad 35.011 \quad 84.369$ TN, ISABELLA

880715131528.9235 .662 TN, MEADOW

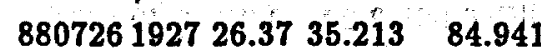
TN, SOUTH CLEVELAND

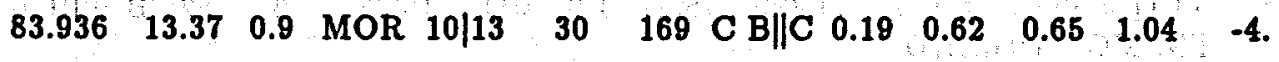

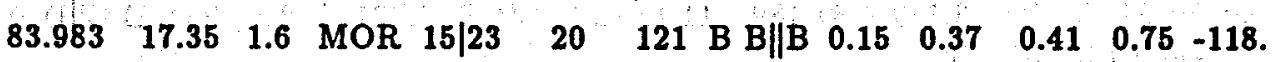

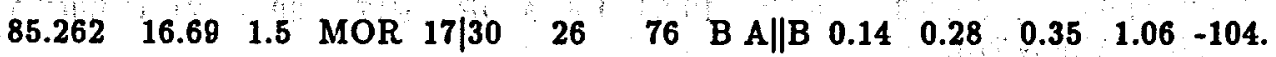

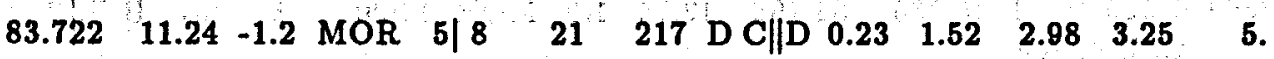

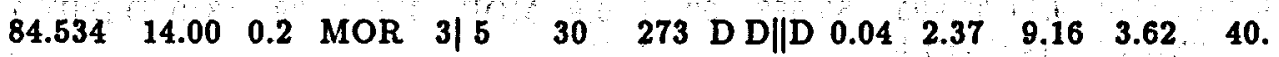

\begin{tabular}{lllll|llllllllllll}
84.390 & 13.48 & 0.3 & MOR & 4 & 6 & 7 & 344 & $\mathrm{D}$ & $\mathrm{D}|| \mathrm{D}$ & 0.16 & 1.45 & 4.66 & 2.07 & 39.
\end{tabular}

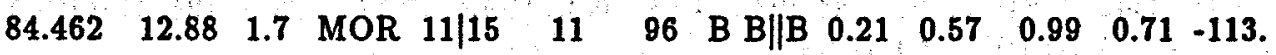

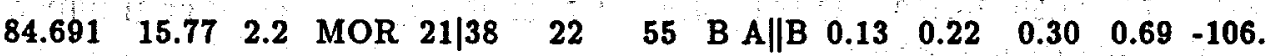

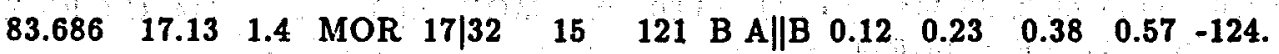

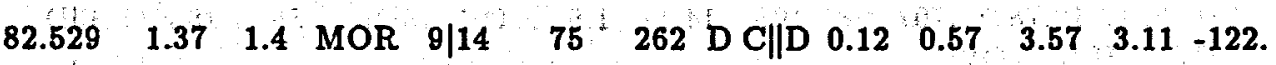

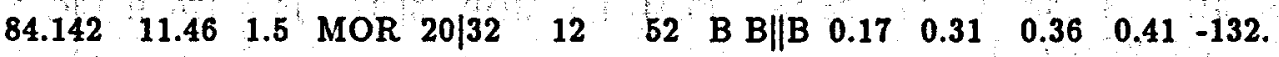

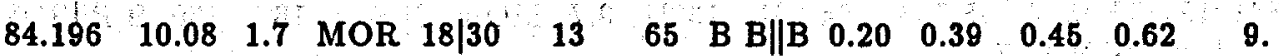

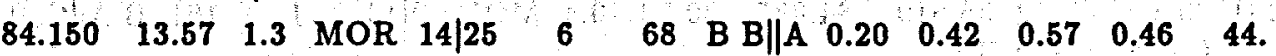

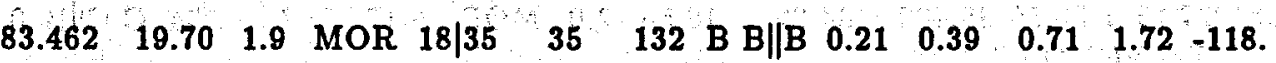

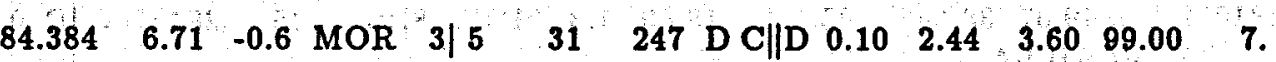

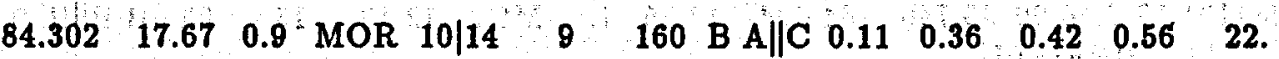

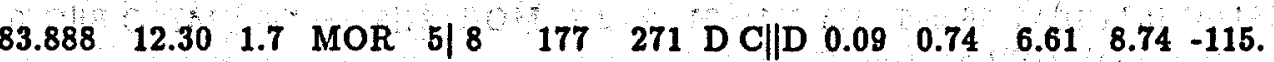

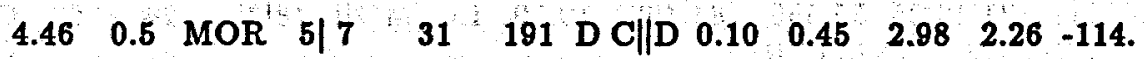

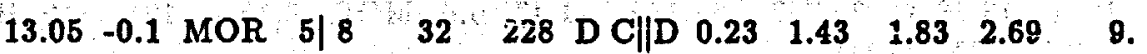

$\begin{array}{lllllllllllllllllllll}13.97 & 0.6 & \text { MOR } & 9 / 16 & 6 & 79 & \text { A Al|A } & 0.13 & 0.41 & 0.46 & 0.38 & -76\end{array}$

$\begin{array}{llllllllllllllllllllll}18.96 & 2.2 & \text { MOR } & 24 \mid 43 & 5 & 69 & \text { B } & \text { B }|| A & 0.17 & 0.29 & 0.35 & 0.54 & -101\end{array}$ 
DATE ORGIN LAT ${ }^{\circ} \mathrm{N}$ LONG ${ }^{\circ} \mathrm{W}$ DEP MAG MOD ST|PH DMIN GAP Q SQD RMS ERH1 ERH2 ERZ AZM

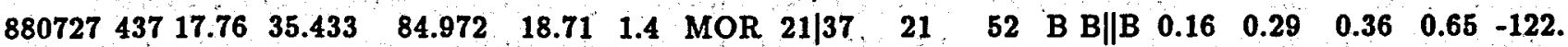

TN, BIG SPRING

$\begin{array}{lllllllllllllllllll}880729 & 233 & 0.85 & 34.819 & 85.203 & 15.00 & 1.1 & \text { MOR } & 10 \mid 17 & 22 & 145 & \text { C B B } & 0.17 & 0.52 & 0.74 & 1.70 & -119 .\end{array}$ GA, NICKAJACK GAP

88080765441.3735 .283
TN, BIRCHWOOD

88081417645.4135 .178

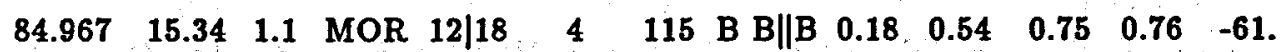

TN, MC FARLAND

$88082034853.76 \quad 36.739$

VA, ELK CREEK

$88082322028.35 \quad 36.383$

TN, TAZEWELL

$88091053519.62 \quad 35.236$ NC, UNAKA

880915224059.7135 .905 TN, BOYDS CREEK

$880915234059.0735 .308 \quad 84.211$
TN, BALD RIVER FALLS

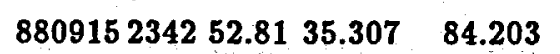

TN, BALD RIVER FALLS

$881001 \quad 749 \quad 43.50 \quad 36.138 \quad 83.894$ TN, BIG RIDGE PARK

$881004115523.72 \quad 35.364 \quad 84.241$ TN, BALD RIVER FALLS

$8810142217 \quad 12.78 \quad 34.707 \quad 85.106$ GA, VILLANOW

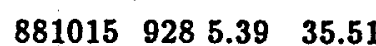
TN, VONORE

$88101985646.47 \quad 35.506$ TN, VONORE

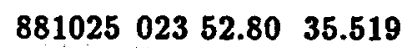
TN, EVENSVILLE

881027153753.5635 .600 TN, VONORE

$88102924549.07 \quad 36.648$ KY, VARILLA

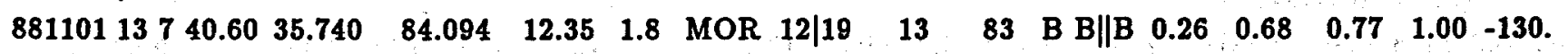
TN, BINFIELD

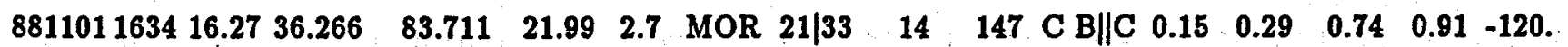
TN, POWDER SPRINGS

$\begin{array}{lllllllllllllllllllllll}881117 & 721 & 46.05 & 35.555 & 84.462 & 10.39 & 1.6 & \text { MOR } & 18 & 35 & 24 & 62 & \text { C } & \text { B } \| C & 0.23 & 0.37 & 0.52 & 0.78 & -124\end{array}$ TN, SWEETWATER

88120191828.4135 .762 TN, CONCORD

$881204300.94 \quad 35.544$ TN, SWEETWATER

88122222446.9836 .110

$\begin{array}{lllllllllllllll}84.159 & 11.47 & 0.6 & \text { MOR } & 8 & 13 & 12 & 112 & \text { B A } & \text { A } & 0.14 & 0.45 & 0.59 & 0.72 & 6\end{array}$

$\begin{array}{lllllllllllllll}84.379 & 14.44 & 0.0 & \text { MOR } & 8 & 12 & 20 & 144 & \text { B A } & \text { AlC } & 0.11 & 0.32 & 0.60 & 0.47 & 40 .\end{array}$ TN, JOHN SEVIER

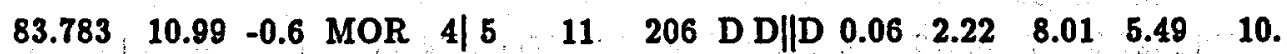

\begin{tabular}{lllll|llllllllll}
84.141 & 10.94 & 1.0 & MOR & 5 & 8 & 18 & 170 & B A & AC & 0.04 & 0.20 & 0.37 & 0.68 & -101
\end{tabular}

$\begin{array}{llllllllllllllll}84.161 & 13.21 & 0.7 & \text { MOR } & 9 \mid 14 & 18 & 130 & \text { B } & \text { B } & \text { B } & 0.29 & 1.02 & 1.55 & 1.74 & 38 .\end{array}$

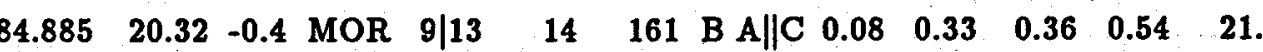

$\begin{array}{llllllllllllllll}84.220 & 16.58 & 2.0 & \text { MOR } & 9 \mid 17 & & 7 & 134 & \text { C C }|| B & 0.33 & 1.07 & 1.42 & 1.44 & -128 .\end{array}$

$\begin{array}{llllllllllllll}83.562 & 18.69 & 1.3 & \text { MOR } & 8 \mid 10 & 45 & 284 & \text { D C } & \text { C D } & 0.09 & 0.57 & 1.46 & 3.35 & -109 .\end{array}$

$\begin{array}{lllllllllllllll}84.499 & 15.47 & 1.8 & \text { MOR } & 18 \mid 28 & 16 & 52 & \text { B } & \text { B } \| \text { B } & 0.21 & 0.50 & 0.65 & 1.08 & 44 .\end{array}$

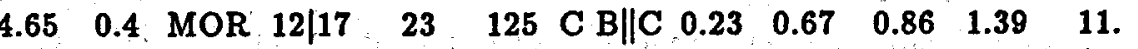

$\begin{array}{lllllllllllll}4.74 & 1.7 & \text { MOR } & 9 \mid 16 & 8 & 133 & \text { B } & \text { B }|| B & 0.15 & 0.41 & 1.06 & 0.88 & -12 .\end{array}$

\begin{tabular}{llll|lllllllllll}
5.80 & 0.1 & MOR & 5 & 8 & 20 & 114 & C C & CC & 0.16 & 0.47 & 1.03 & 36.51 & -5.
\end{tabular}

$\begin{array}{lllllllllllll}14.11 & 1.5 & \text { MOR } & 15 \mid 25 & 35 & 188 & \mathrm{C} & \mathrm{B} \| \mathrm{D} & 0.22 & 0.58 & 1.02 & 0.93 & -104\end{array}$ 
DATE ORGIN LAT $^{\circ} \mathrm{N}$ LONG ${ }^{\circ} \mathrm{W}$ DEP MAG MOD ST|PH DMIN GAPQ SQD RMS ERH 1 ERH2 ERZ AZM $\begin{array}{llllllllllllllllllll}890101 & 1027 & 53.92 & 35.837 & 84.039 & 14.40 & -0.9 & \text { MOR } & 3 \mid & 6 & 25 & 270 & \text { D C } & 0.07 & 0.07 & 0.85 & 2.66 & 0.93 & -98 .\end{array}$ TN, LOUISVILLE

$\begin{array}{llllllllllllllllllll}890101 & 175 & 56.83 & 35.645 & 84.109 & 10.89 & -0.5 & \text { MOR } & 3 \mid & 5 & 9 & 297 & \text { D C }|| D & 0.06 & 1.60 & 10.49 & 8.93 & -106 .\end{array}$ TN, BINFIELD

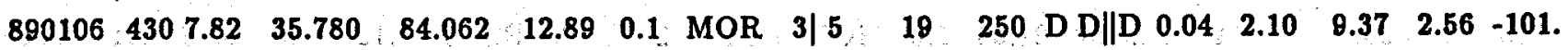
TN, LOUISVILLE $89011842535.77 \quad 36.005$ TN, WINDROCK 890119113825.7135 .658
TN, GATLINBURG $89012175443.32 \quad 36.378$. TN, BLUFF CITY

$890123102811.9335 .206,84.779$ TN, EAST CLEVELAND

$89021544619.65 \quad 35.486 \quad 85.032 \quad 18$ TN, GRAYSVILLE

$\begin{array}{lllll}890226 & 034 & 6.10 & 36.329 & 82.132\end{array}$ TN, ELIZABETHTON $\begin{array}{lllllll}890227 & 5 & 5 & 5.57 & 35.774 & 83.957 & 16\end{array}$ TN, MARYVILLE

$8903049283.22 \quad 35.409 \cdot 84.509$ TN, ATHENS

$890306195721.5035 .806 \quad 83.965$ TN, MARYVILLE $890307 \quad 10225.68 \quad 35.480 \quad 84.132$
TN, RAFTER

\begin{tabular}{l}
$8903101052 \quad 22.66 \quad 35.168 \quad 84.600$ \\
\hline
\end{tabular} TN, OSWALD DOME \begin{tabular}{l}
$890313 \quad 12448.69 \quad 35.571$ \\
\hline
\end{tabular} TN, SWEETWATER

$890316 \quad 043 \quad 58.27 \quad 35.326$ TN, CALHOUN

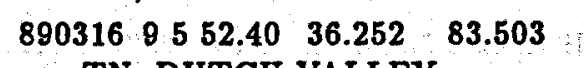
84.45217 $\begin{array}{llllllllllllllllll}26.50 & 1.4 & \text { MOR } & 4 & 5 & 38 & 360 & \text { D D } & \text { |D } & 0.02 & 2.68 & 5.01 & 7.00 & -86\end{array}$ TN, DUTCH VALLEY 890401221523.5434 .987 GA, EAST RIDGE 890402193245.9435 .466 TN, ENGLEWOOD $89040310137.20 \quad 36.082$ TN, NEW MARKET 890413111857.4836 .069 TN, JOHN SEVIER 89041616232.7135 .592 TN, VONORE $890420152547.83 \quad 35.276$ TN, CHARLESTON 890429193432.7536 .115 NC, HUNTDALE

\subsection{0}

84.484

83.617

83.7

84.134

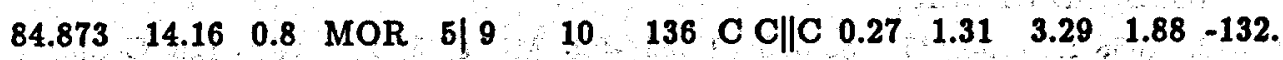

$\begin{array}{lllllllllllllllll}82.351 & 5.70 & 1.7 & \text { MOR } & 13 \mid 20 & 42 & 89 & \text { C C }|| C & 0.41 & 0.88 & 0.95 & 6.08 & 20\end{array}$ 
DATE ORGIN LAT $^{\circ} \mathrm{N}$ LONG ${ }^{\circ} W$ DEP MAG MOD ST|PH DMIN GAP Q SQD RMS ERH1 ERH2 ERZ AZM

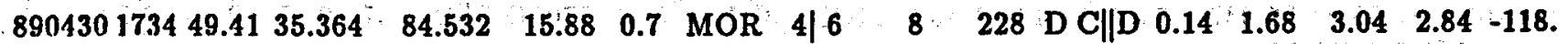
TN, ETOWAH

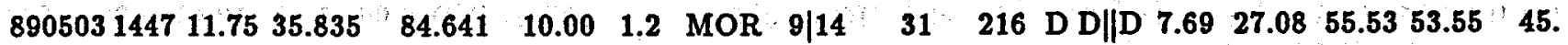
TN, ROCKWOOD

$\begin{array}{lllllllllllllllllllllllll}890504 & 633 & 29.80 & 35.702 & 84.506 & 21.05 & 1.0 & \text { MOR } & 8 & 14 & 27 & 106 & \text { B B B } & 0.12 & 0.43 & 0.63 & 1.19 & -135 .\end{array}$ TN, PATTIE GAP

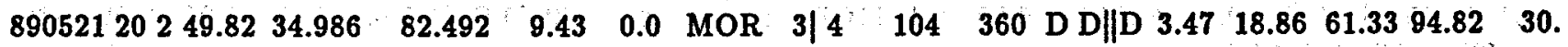
SC, GREENVILLE

$\begin{array}{lllllllllllllllllllllll}890524 & 312 & 55.21 & 35.524 & 84.096 & 13.55 & -0.4 & \text { MOR } & 4 \mid 6 & 19 & 269 & \text { D C } & 0.18 & 1.82 & 5.00 & 6.86 & -125 .\end{array}$ TN, TALLASSEE

$\begin{array}{lllllllllllllllll}890525 & 1327 & 54.26 & 35.478 & 84.256 & 15.41 & 0.6 & \text { MOR } & 7 \mid 10 & 21 & 216 & \text { C B B } & 0.10 & 0.38 & 0.85 & 1.09 & 35 .\end{array}$ TN, MT VERNON

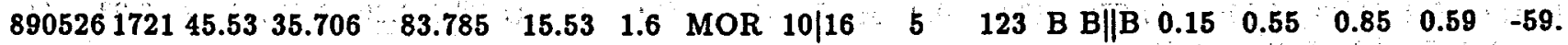
TN, KINZEL SPRINGS

$\begin{array}{lllllllllllllllllllll}890527 & 49 & 7.71 & 36.340 & 82.306 & 1.47 & 2.0 & \text { MOR } & 17 / 25 & 20 & 132 & \text { C C }|| C & 0.43 & 0.84 & 1.62 & 2.85 & -83\end{array}$ TN, JOHNSON CITY

$\begin{array}{llllllllll}890531 & 644 & 24.58 & 35.120 & 84.900 & 14.72 & 1.0 & \text { MOR } & 12 / 18 & 16\end{array}$ TN, MCDONALD

$\begin{array}{llllllllllllllllllllll}890603 & 1519 & 39.78 & 35.948 & 84.054 & 13.50 & -0.2 & \text { MOR } & 4 / 8 & 16 & 135 & \text { C B } & \text { BC } & 0.10 & 0.45 & 0.74 & 1.22 & -99 .\end{array}$ TN, BEARDEN

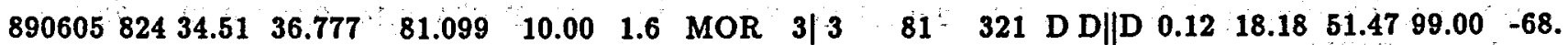
VA, CRIPLE CREEK

$890605114336.0036 .046 \quad 81.158$ NC, MORAVIAN FALLS

$\begin{array}{llllllllll}890607 & 637 & 52.27 & 35.415 & 84.581 & 9.00 & -0.1 & \text { MOR } & 3 / 5 & 15\end{array}$ TN, ATHENS

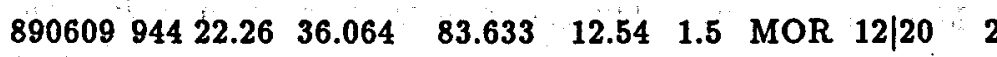
TN, MASCOT

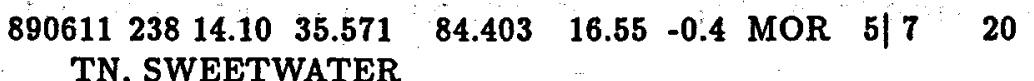

$\begin{array}{lllllllllllllllll}890613 & 232 & 29.27 & 36.684 & 83.652 & 22.83 & 2.0 & \text { MOR } & 16 \mid 28 & 9 & 208 & \text { C B B } & 0.20 & 0.55 & 1.17 & 0.82 & -90 .\end{array}$ KY, MIDDLESBORO NORTH

$\begin{array}{lllllllllllllllllllll}890613 & 721 & 5.80 & 35.972 & 83.841 & 9.81 & -1.2 & \text { MOR } & 6 / 9 & 6 & 170 & \text { B A } & \text { AC } & 0.06 & 0.27 & 0.57 & 0.38 & 7 .\end{array}$ TN, SHOOKS GAP

$\begin{array}{llllllllll}890615 & 6 & 9 & 2.45 & 36.360 & 83.750 & 18.41 & 0.6 & \text { MOR } & 9 \mid 16 \quad 20\end{array}$ TN, MAYNARDVILLE

$\begin{array}{lllllllllllllllllll}890618 & 3 & 0 & 20.63 & 35.945 & 82.490 & 4.26 & 1.9 & \text { MOR } & 16 \mid 24 & 29 & 110 & \text { C B BC } & 0.27 & 0.55 & 1.14 & 1.44 & -68 .\end{array}$ NC, BALD CREEK

$\begin{array}{lllllllllllllllllllllll}890620 & 843 & 37.80 & 35.256 & 82.455 & 4.68 & 2.0 & \text { MOR } & 16 \mid 23 & 19 & 165 & \text { C C } \| \mathrm{C} & 0.36 & 0.82 & 2.09 & 2.10 & -107 .\end{array}$ NC, HENDERSONVILLE

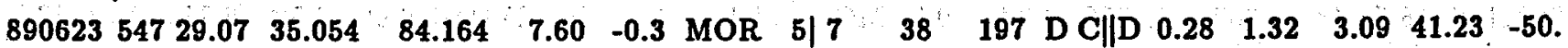
NC, PERSIMMON CREEK

$\begin{array}{llllllllllllllllllllllll}890701 & 13 & 0 & 58.29 & 35.935 & 82.499 & 12.83 & 0.8 & \text { MOR } & 4 & 7 & 29 & 166 & \text { C C } & \text { |C } & 0.26 & 1.60 & 2.81 & 2.38 & -89\end{array}$ NC, BALD CREEK

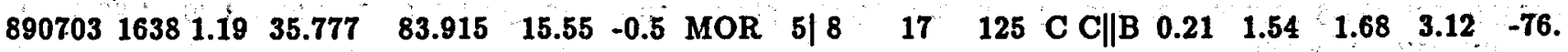
TN, MARYVILLE

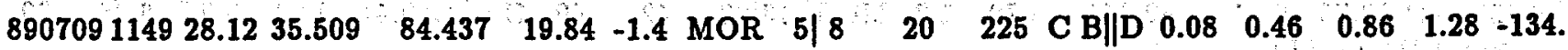
TN, SWEETWATER 
DATE ORGIN LAT $^{\circ} \mathrm{N}$ LONG ${ }^{\circ} W$ DEP MAG MOD ST/PH DMIN GAP Q SQD RMS ERH1 ERH2 ERZ AZM 890709115157.7335 .527
TN, SWEETWATER $89070911575.90 \quad 35.520$

TN, SWEETWATER

890709153032.1034 .789 SC, OLD PICKENS

890711145328.5435 .53

TN, SWEETWATER

$89071763944.27 \quad 35.520$ TN, MADISONVILLE

$\begin{array}{rr}890718 \quad 334 & 26.78 \quad 35.089 \\ \text { TN, EAST CHATTANOOGA }\end{array}$

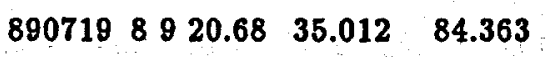

TN, ISABELLA

$89071913583.26 \quad 36.036$

TN, NEW MARKET

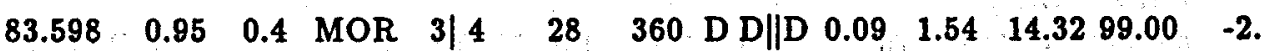

$8907255545.69 \quad 35.597$

TN, DECATUR

890730203859.6536 .041

TN, FOUNTAIN CITY

$89073105726.65 \quad 36.453$.

TN, LA FOLLETTE

$890805 \quad 526 \quad 37.94 \quad 35.008$

TN, ISABELLA

89080894211.5735 .965

TN, BEARDEN

\begin{tabular}{l}
$890809 \quad 926 \quad 0.49 \quad 35.327$ \\
\hline
\end{tabular}

TN, MECCA

89081215716.3134 .747
AL, SCOTTSBORO

890815124632.4835 .269

TN, CHARLESTON

890820225425.3635 .913

TN, LOVELL

$890825 \quad 53836.15 \quad 35.472$.

TN, RICEVILLE

890827235154.9336 .090

TN, PETROS

890830104215.0635 .654

TN, MELVINE

89090395733.7335 .533

TN, SWEETWATER

$\begin{array}{lllllllllllllll}84.871 & 22.49 & 0.6 & \text { MOR } & 12 \mid 20 & 29 & 74 & \text { B A } & \text { A B } & 0.12 & 0.33 & 0.39 & 1.01 & -4 .\end{array}$

$\begin{array}{lllllllllllllll}83.942 & 25.07 & -0.2 & \text { MOR } & 7 \mid 12 & 20 & 133 & \text { B } & \text { A } \| \text { B } & 0.10 & 0.43 & 0.58 & 0.88 & 7\end{array}$

$\begin{array}{llllllllllllll}84.070 & 9.25 & 0.9 & \text { MOR } & 10 \mid 12 & 34 & 341 & \text { C A A |D } & 0.09 & 0.33 & 0.65 & 0.68 & -67 .\end{array}$

\begin{tabular}{lllll|llllllllllll}
84.373 & 5.91 & 0.2 & MOR & $3 \mid$ & 6 & 32 & 133 & C C $\| \mathrm{C}$ & 0.16 & 0.42 & 0.52 & 37.02 & -37
\end{tabular}

$\begin{array}{lllllllllllllll}84.068 & 15.67 & 0.4 & \text { MOR } & 8 \mid 12 & 18 & 258 & \text { C A } & \text { AD } & 0.07 & 0.32 & 0.40 & 0.76 & 25 .\end{array}$

$\begin{array}{lllllllllllllllll}84.376 & 9.39 & 1.2 & \text { MOR } & 10 \mid 16 & 7 & 113 & \text { B } & \text { B } & \text { ||B } & 0.27 & 0.84 & 1.06 & 1.41 & -125\end{array}$

\begin{tabular}{lllll|llllllllll}
86.076 & 0.50 & 1.7 & MOR & 5 & 7 & 160 & 213 & D C & 1 D & 0.23 & 0.50 & 2.67 & 2.66 & -102.
\end{tabular}

$\begin{array}{lllllllllllllll}84.872 & 14.66 & 1.1 & \text { MOR } & 6 / 11 & 10 & 130 & \mathrm{C} & \mathrm{C} \| \mathrm{B} & 0.16 & 0.65 & 1.84 & 1.06 & 35 .\end{array}$

$\begin{array}{llllllllllllllll}84.163 & 12.80 & 1.7 & \text { MOR } & 14 \mid 24 & 13 & 69 & \text { A A } & \text { A A } & 0.13 & 0.33 & 0.37 & 0.33 & -52\end{array}$

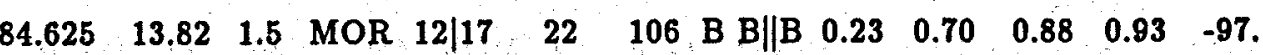

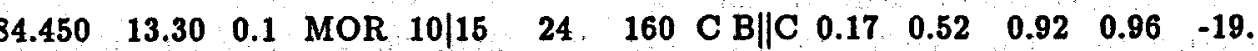

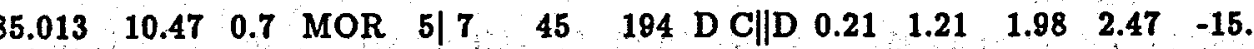

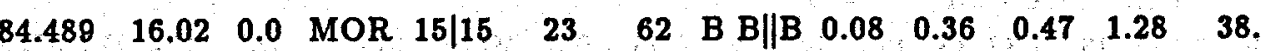

$8909075186.29 \quad 35.499$

$\begin{array}{llllllllllllll}84.431 & 20.47 & 3.1 & \text { MOR } & 19 \mid 31 & 19 & 69 & \text { A A A A } & 0.12 & 0.24 & 0.35 & 0.57 & 43\end{array}$

TN, ENGLEWOOD

890916162632.5635 .524

$\begin{array}{llllllllllllllllll}84.021 & 13.96 & -0.7 & \text { MOR } & 4 \mid 7 & 23 & 185 & \text { C B } & \text { B } & 0.05 & 0.29 & 1.00 & 1.39 & -101 .\end{array}$

TN, TALLASSEE

89091716811.1035 .796

TN, WALDEN CREEK 
DATE ORGIN LAT ${ }^{\circ} \mathrm{N}^{\circ} \mathrm{NG}^{\circ} \mathrm{W}$ DEP MAG MOD ST|PH DMIN GAP Q SQD RMS ERH1 ERH2 ERZ AZM

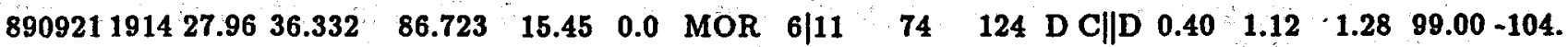

TN, GOODLETTSVILLE

\subsection{TN, MECCA}

890925212939.4534 .978 SC, TAMASSEE

$89100210313.35 \quad 35.521$

NC, CANTON

891003233818.8035 .583

TN, NIOTA

891004181617.6135 .534

TN, VONORE

8910059430.4935 .376 TN, ATHENS

$891008 \quad 12549.05 \quad 36.376$ NC, WHITEHEAD

891025183742.0335 .328 NC, WESSER

$891027 \quad 73947.75 \quad 35.746$ TN, BLOCKHOUSE

$89110441420.64 \quad 35.639$ TN, MEADOW

891112121810.2136 .781 KY, BALKAN

$89120205941.74 \quad 35.714$ TN, BINFIELD

891202133145.6435 .988 TN, SHOOKS GAP

89120218227.6636 .610 TN, EAGAN

89121584745.0135 .900 TN, SHOOKS GAP

891217184032.8135 .055 NC, REID

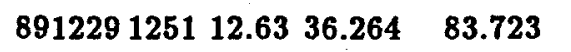
TN, POWDER SPRINGS

$900102201724.58 \quad 35.093 \quad 82.969$ NC, REID

900119173652.7034 .957 GA, COHUTTA

900128113823.3735 .217 TN, FARNER

900202111435.8435 .656
TN, LOUDON

90020521053.8335 .925 TN, NEWPORT

$900208 \quad 55331.48 \quad 35.546$ TN, TALLASSEE

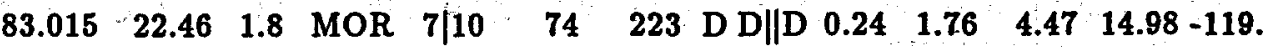

\begin{tabular}{lllll|llllllllllll}
82.867 & 12.09 & 0.8 & MOR & 4 & 5 & 21 & 155 & D C & D & 0.09 & 1.79 & 5.03 & 5.07 & -127.
\end{tabular}

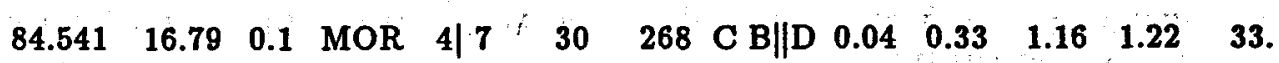

$\begin{array}{llllllllllllllll}84.226 & 16.46 & 1.3 & \text { MOR } & 6 \mid 11 & 15 & 143 & \mathrm{C} & \mathrm{B} \| \mathrm{C} & 0.11 & 0.44 & 0.86 & 1.11 & -127 .\end{array}$

$\begin{array}{lllllllllllllllll}84.623 & 16.01 & 0.7 & \text { MOR } & 4 & 7 & 16 & 282 & \text { D D } & \text { || } & 0.35 & 3.67 & 6.14 & 4.84 & -39 .\end{array}$

$\begin{array}{lllllllllllllll}81.206 & 5.84 & 1.0 & \text { MOR } & 4 & 7 & 32 & 166 & \text { C C } \| C & 0.19 & 0.61 & 1.64 & 71.29 & 4 .\end{array}$

\begin{tabular}{lllll|llllllllll}
83.617 & 4.50 & 1.6 & MOR & 5 & 9 & 7 & 169 & C C & C C & 0.15 & 0.80 & 3.48 & 2.19 & -2.
\end{tabular}

$\begin{array}{lllllllllllllll}83.977 & 12.70 & 0.7 & \text { MOR } & 4 & 6 & 21 & 230 & \text { D } & \text { D }|| D & 0.10 & 0.83 & 4.14 & 1.78 & -87 .\end{array}$

$\begin{array}{llllllllllllllll}84.157 & 13.26 & -0.3 & \text { MOR } & 4 & 7 & 6 & 185 & \text { D D } & \text { D } & 0.10 & 0.91 & 2.97 & 1.86 & -118 .\end{array}$

$\begin{array}{lllllllllllllll}83.520 & 19.83 & 1.8 & \text { MOR } & 10 \mid 15 & 24 & 264 & \text { C } & \text { B } & \text { DD } & 0.10 & 0.58 & 1.25 & 0.94 & -73 .\end{array}$

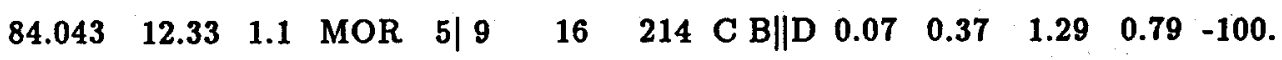

$\begin{array}{llllllllllllll}83.844 & 12.73 & 2.9 & \text { MOR } & 19 / 27 & 23 & 45 & \text { B A } & \text { A B } & 0.15 & 0.34 & 0.46 & 0.51 & 16 .\end{array}$

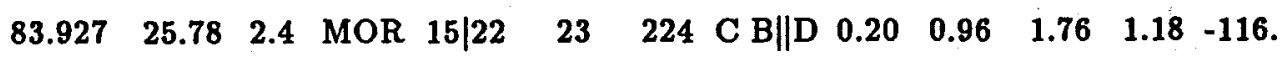

$\begin{array}{lllllllllllllll}83.869 & 26.61 & 1.3 & \text { MOR } & 3 & 5 & 28 & 179 & \text { D D } & \text { |D } & 0.06 & 2.70 & 11.96 & 12.85 & -99 .\end{array}$

\begin{tabular}{lllll|lllllllll}
82.949 & 12.73 & 1.3 & MOR & 4 & 6 & 34 & 186 & D D $\mid$ D & 0.26 & 3.91 & 11.82 & 11.92 & -96.
\end{tabular}

$\begin{array}{lllllllllllll}12.90 & 1.1 & \text { MOR } & 6 / 11 & 50 & 221 & \mathrm{D} & \mathrm{C} \| \mathrm{D} & 0.07 & 0.70 & 11.84 & 19.17 & -74\end{array}$

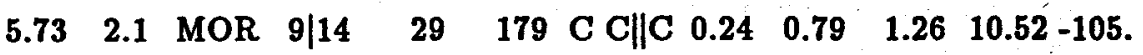

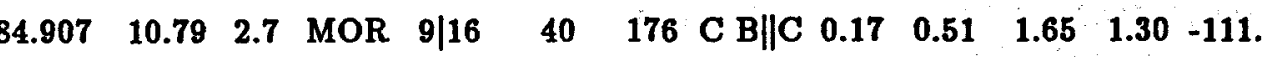

$\begin{array}{lllllllllllllll}84.313 & 9.11 & 0.5 & \text { MOR } & 3 \mid 4 & 18 & 184 & \text { D D } & \text { D } & 0.10 & 6.70 & 10.20 & 18.55 & -49\end{array}$

$\begin{array}{llllllllllllll}84.264 & 13.83 & 2.2 & \text { MOR } & 8 \mid 14 & 5 & 148 & \text { B A } & \text { A } & 0.12 & 0.44 & 0.65 & 0.37 & -108\end{array}$

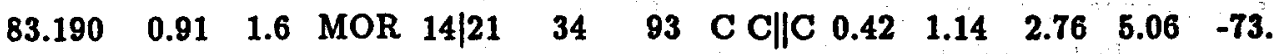

$\begin{array}{llllllllllllllll}84.010 & 12.15 & -1.3 & \text { MOR } & 4 \mid 7 & 23 & 179 & \text { C C } & \mathbf{C} & 0.26 & 1.49 & 4.02 & 10.95 & -80 .\end{array}$ 
DATE ORGIN LAT ${ }^{\circ}$ LONG ${ }^{\circ} W$ DEP MAG MOD ST|PH DMIN GAP Q SQD RMS ERH1 ERH2 ERZ AZM $\begin{array}{lllllllllllllllllllllll}900210 & 2342 & 7.89 & 35.269 & 84.068 & 8.51 & 2.5 & \text { MOR } & 15 \mid 28 & 36 & 79 & \text { C } & \text { B } \| \text { C } & 0.26 & 0.50 & 0.57 & 1.73 & -48 .\end{array}$ TN, BIG JUNCTION 900214104338.4735 .565 NC, SILERS BALD

900217163032.1135 .713 TN, MEADOW

$900222 \quad 654.2 .80 \quad 36.349$ NC, TODD

900223171044.8335 .513 TN, SWEETWATER

900223171253.6035 .509 TN, SWEETWATER $900301 \quad 4414.88 \quad 36.092$ TN, JOHN SEVIER

$900303,858.28 .94 \quad 35.98$ TN, SHADY GROVE 900303222759.8235 .306 NC, HORSE SHOE

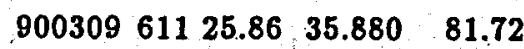
NC, COLLETTSVILLE

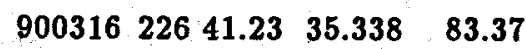
NC, ALARKA

\begin{tabular}{l}
$90031834134.95 \quad 35.671$ \\
\hline
\end{tabular} TN, LOUDON

$900320161055.76 \quad 36.009 \quad 83.443$ TN, JEFFERSON CITY

$90032194439.39 \quad 34.866$ GA, TUNNEL HILL

$900324 \quad 65147.66 \quad 35.148$ TN, BENTON "

$9003251347 \quad 16.0635 .192$ NC, UNAKA

9003263719.8135 .551 TN, SWEETWATER $90033155630.40 \quad 35.304$ NC, HORSE SHOE

900331162744.4035 .933 TN, HARRIMAN

900419203648.4035 .944 TN, BEARDEN

900510233832.8835 .243 TN, FARNER

90051113157.3935 .022 NC, CASHIERS

B005141133 36.7435 .443 TN, ATHENS

$900518 \quad 927 \quad 27.10 \quad 34.982$ SC, SALEM

$\begin{array}{llllllllllllllll}85.068 & 10.59 & 2.0 & \text { MOR } & 6 \mid 10 & 28 & 205 & \text { C B } & \text { ||D } & 0.11 & 0.46 & 1.16 & 1.39 & -83\end{array}$ $\begin{array}{llllllllllllllll}84.646 & 17.62 & 2.0 & \text { MOR } & 6 \mid 12 & 26 & 148 & \text { C } & B \| C & 0.17 & 0.60 & 1.07 & 1.72 & 42 .\end{array}$ $\begin{array}{lllllllllllllll}84.143 & 11.20 & 1.2 & \text { MOR } & 5 \mid 10 & 32 & 95 & \text { C C } & \text { |C } & 0.21 & 0.85 & 1.08 & 6.74 & -84\end{array}$ \begin{tabular}{lllll|lllllllllllll}
84.404 & 14.79 & 1.1 & MOR & 4 & 5 & 25 & 360 & D C C & & 0.10 & 2.17 & 6.66 & 18.33 & -72.
\end{tabular} $\begin{array}{llllllllllllllll}82.520 & 5.88 & 0.8 & \text { MOR } & 6 \mid 10 & 25 & 149 & \mathrm{C} & \mathrm{C} \| \mathrm{C} & 0.18 & 0.53 & 1.21 & 5.06 & -87\end{array}$

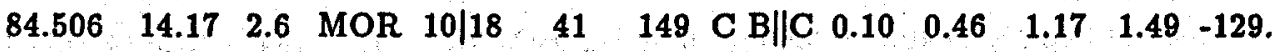

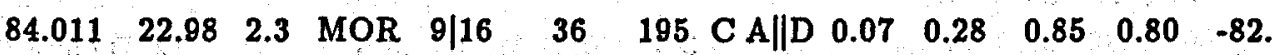
$\begin{array}{llllllllllllllll}84.359 & 11.92 & -0.4 & \text { MOR } & 4 & 7 & 13 & 198 & \text { D C } & 0.13 & 0.75 & 36.64 & 25.68 & 25 .\end{array}$ $\begin{array}{llllllllllllllll}83.019 & 7.79 & 1.1 & \text { MOR } & 12 \mid 17 & 37 & 189 & \text { C } & \text { B } & \text { |D } & 0.21 & 0.64 & 1.07 & 1.72 & -109 .\end{array}$

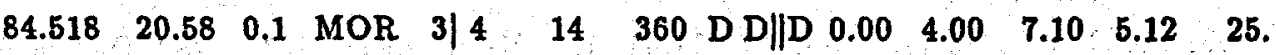

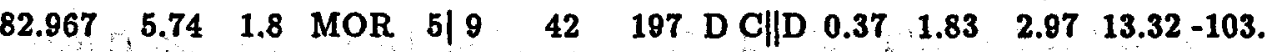


DATE ORGIN LAT $^{\circ} \mathrm{N}$ LONG ${ }^{\circ}$ W DEP MAG MOD ST|PH DMIN GAP Q SQD RMS ERH1 ERH2 ERZ AZM

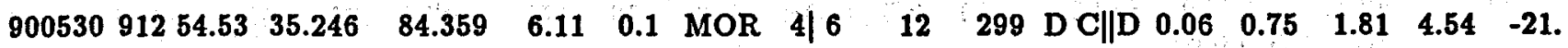

TN, FARNER

900605225046.1335 .552

TN, TALLASSEE

900606114651.5334 .527

SC, LA FRANCE

90061118724.7235 .533

NC, SILERS BALD

90061311158.3134 .861

GA, KENSINGTON

$900616 \quad 2300.14 \quad 35.424$

TN, BRAYTON

900618 \& $157.50 \quad 35.005$

TN, ISABELLA

$90062132132.28 \quad 34.948$

GA, CULBERSON

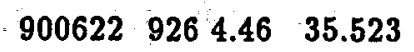

TN, VONORE

90062411116.0535 .808

TN, LOUISVILLE

$900708 \quad 14587.54 \quad 36.503$

TN, COLEMAN GAP

900711184110.8035 .795

TN, MARYVILLE

$90071383113.32 \quad 35.014$

TN, ISABELLA

900714234829.2935 .683

TN, LOUDON

$900721133250.06 \quad 36.427 \quad 82.078$

TN, CARTER

90072811939.2434 .933
GA, RINGGOLD

$900729141942.4135 .222 \div 84.821$ TN, EAST CLEVELAND

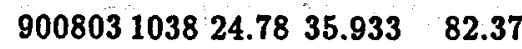
NC, BALD CREEK

$900804111648.15 \quad 36.402 \quad 83.606$ TN, TAZEWELL

$900808123055.8035 .865 \quad 81.748$ NC, MORGANTON NORTH

$900810 \quad 555 \quad 37.75 \quad 35.023 \quad 84.357$ TN, ISABELLA

$900810 \quad 55915.46 \quad 35.031 \quad 84.385$ TN, DUCKTOWN

$9008161020 \quad 40.2935 .413$ TN, BRAYTON

90081721116.6536 .891 KY, BLEDSOE $\begin{array}{llllllllllllllllll}84.059 & 6.46 & -1.1 & \text { MOR } & 4 / 6 & 19 & 170 & \mathrm{C} & \mathrm{C} \| \mathrm{C} & 0.04 & 0.47 & 1.16 & 7.50 & -103 .\end{array}$

$\begin{array}{lllllllllllllll}82.797 & 0.42 & 1.7 & \text { MOR } & 8 \mid 10 & 94 & 251 & \text { D C } & \text { D } & 0.17 & 0.66 & 3.50 & 3.83 & -108 .\end{array}$

$\begin{array}{lllllllllllllllll}83.534 & 15.15 & 1.1 & \text { MOR } & 5 & 8 & 26 & 256 & \text { D C } & \text { D } & 0.12 & 0.79 & 1.30 & 2.75 & 26 .\end{array}$

\begin{tabular}{lllll|llllllllllll}
85.332 & 10.56 & 1.6 & MOR & 5 & 8 & 13 & 264 & D C & || & 0.20 & 2.35 & 4.02 & 2.27 & -45
\end{tabular}

$\begin{array}{lllllllllllllll}85.209 & 13.91 & 1.1 & \text { MOR } & 6 \mid 11 & 51 & 193 & \text { C B } & \text { ||D } & 0.09 & 0.39 & 0.66 & 1.69 & 21 .\end{array}$

$\begin{array}{lllllllllllllll}84.360 & 5.79 & 0.9 & \text { MOR } & 5 & 8 & 32 & 230 & \text { D C } & \text { D } & 0.20 & 0.55 & 0.62 & 49.87 & 39 .\end{array}$

$\begin{array}{llllllllllllll}84.195 & 5.87 & 1.0 & \text { MOR } & 6 \mid 10 & 36 & 155 & \mathrm{C} & \mathrm{C} \| \mathrm{C} & 0.24 & 0.90 & 1.57 & 99.00 & -80\end{array}$

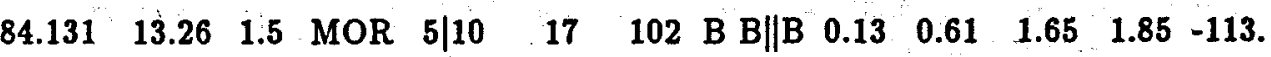

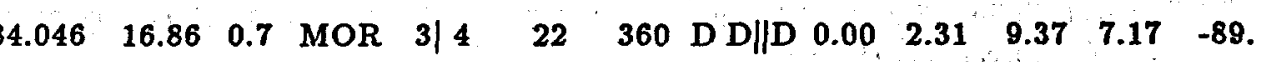

$\begin{array}{llllllllllllllll}83.421 & 10.83 & 1.5 & \text { MOR } & 5 & 7 & 84 & 282 & \text { D C } & 0 \text { D } & 0.10 & 1.19 & 5.62 & 9.71 & -90 .\end{array}$

$\begin{array}{lllllllllllllllll}15.60 & 2.8 & \text { MOR } & 14 \mid 23 & 24 & 104 & \text { B A } \| \text { B } & 0.12 & 0.35 & 0.44 & 0.90 & -107\end{array}$

$\begin{array}{lllllllllllllll}6.42 & 1.7 & \text { MOR } & 5 & 8 & 32 & 132 & \mathbf{C} & \mathrm{C} \| \mathrm{C} & 0.18 & 1.16 & 1.46 & 57.94 & -37 .\end{array}$

$\begin{array}{llllllllllllll}18.04 & 1.1 & \text { MOR } & 5 & 9 & 14 & 163 & \mathrm{C} & \mathrm{B} \| \mathrm{C} & 0.10 & 0.59 & 0.73 & 0.96 & -81\end{array}$

$\begin{array}{lllllllllllll}9.37 & 2.1 & \text { MOR } & 7 \mid 12 & 43 & 205 & \mathrm{C} & \mathrm{B}|| \mathrm{D} & 0.15 & 1.14 & 1.34 & 1.42 & -119 .\end{array}$

$\begin{array}{llllllllllllll}8.85 & 2.9 & \text { MOR } & 13 \mid 22 & 31 & 168 & \mathrm{C} & \mathrm{B} \| \mathrm{C} & 0.15 & 0.37 & 0.80 & 0.87 & -81\end{array}$

$\begin{array}{lllllllllllll}12.48 & 2.5 & \text { MOR } & 10 \mid 17 & 35 & 110 & \text { B A } & \text { AC } & 0.14 & 0.54 & 0.60 & 0.67 & -82 .\end{array}$

$\begin{array}{lllllllllllllll}2.48 & 1.6 & \text { MOR } & 4 & 7 & 23 & 161 & \mathrm{C} & \mathrm{C} \| \mathrm{C} & 0.19 & 1.02 & 4.75 & 8.81 & -62 .\end{array}$

$\begin{array}{llllllllllllll}5.38 & 2.0 & \text { MOR } & 8 \mid 13 & 84 & 273 & \mathrm{C} & \mathrm{B} \mid \mathrm{D} & 0.07 & 0.34 & 1.15 & 1.56 & -104\end{array}$

$\begin{array}{lllllllllllllll}3.58 & 0.8 & \text { MOR } & 3 \mid 5 & 28 & 182 & \text { D C ||D } & 0.11 & 1.44 & 22.67 & 29.25 & -10 .\end{array}$

$\begin{array}{lllllllllllll}7.57 & 0.7 & \text { MOR } & 3 \mid 5 & 34 & 253 & \mathrm{D} C|| D & 0.09 & 2.17 & 3.16 & 57.91 & 20 .\end{array}$

$\begin{array}{lllllllllllllll}12.74 & 1.0 & \text { MOR } & 3 \mid 5 & 32 & 245 & \text { D C } & 5 & 0.15 & 2.50 & 3.34 & 18.58 & 20\end{array}$

$\begin{array}{lllllllllllllll}85.146 & 11.64 & 1.2 & \text { MOR } & 4 & 7 & 51 & 282 & \text { D C } & \text { D } & 0.09 & 0.78 & 2.14 & 1.40 & -30 .\end{array}$

$\begin{array}{lllllllllllllll}83.372 & 3.87 & 3.5 & \text { MOR } & 14 \mid 21 & 119 & 225 & \text { C B } & \text { BD } & 0.14 & 0.56 & 2.13 & 2.30 & -106\end{array}$ 
DATE ORGIN LAT $^{\circ} \mathrm{N}$ LONG ${ }^{\circ}$ W DEP MAG MOD ST|PH DMIN GAP Q SQD RMS ERH1 ERH2 ERZ AZM $\begin{array}{llllllllllllllllll}900819 & 2110 & 33.07 & 35.481 & 84.045 & 11.85 & 1.8 & \text { MOR } & 6 \mid 11 & 25 & 87 & \text { C B } & \text { BC } & 0.12 & 0.45 & 0.78 & 1.38 & -82 .\end{array}$ TN, WHITEOAK FLATS

\begin{tabular}{|c|c|c|c|c|c|c|c|c|c|c|c|c|}
\hline $\begin{array}{c}0082401349.2635 .888 \\
\text { TN, BEARDEN }\end{array}$ & $84: 046$ & 18.45 & 0.4 & MOR & $4 \mid 6$ & 29 & 285 & $\mathrm{D} C \| \mathrm{D} \quad 0.08$ & 0.85 & 2.85 & 3.41 & -88 \\
\hline $\begin{array}{c}800824104618.3435 .086 \\
\text { TN, DUCKTOWN }\end{array}$ & 84.494 & 7.43 & 1.1 & MOR & $4 \mid 7$ & 27 & 154 & C C $\| C \quad 0.08$ & 0.47 & 1.38 & 9.46 & 26. \\
\hline $\begin{array}{c}00828,45510.68 \quad 35.115 \\
\text { TN, PARKSVILLE }\end{array}$ & 84.635 & 15.26 & 1.1 & MOR: & 4) 6 & 28 & 208 & $\mathrm{D} C \| \mathrm{D} \quad 0.09$ & 0.69 & 2.95 & 4.45 & 18. \\
\hline $\begin{array}{l}00830173542.4636 .150 \\
\text { TN, GOBEY }\end{array}$ & 84.557 & 12.43 & 1.5 & MOR & $4 \mid 7$ & 48 & 283 & C B $\| \mathrm{D} \quad 0.06$ & 0.53 & 1.46 & 0.88 & -85. \\
\hline $\begin{array}{l}\text { DO901 } 5412.6135 .530 \\
\text { TN, VONORE }\end{array}$ & 84.146 & 12.44 & 2.3 & MOR & $9 \mid 14$ & 16 & 88 & $\mathrm{~B} A \| \mathrm{B} \quad 0.08$ & 0.27 & 0.42 & 0.58 & -114 \\
\hline $\begin{array}{c}0091014557.0735 .629 \\
\text { TN, PATTIE GAP }\end{array}$ & 84.596 & 16.89 & 0.7 & MOR & $5 \mid 8$ & 35 & 149 & $C B \| C=0.06$ & 0.37 & 0.53 & 2.42 & -52 \\
\hline $\begin{array}{l}0091273841.4036 .055 \\
\text { TN, MASCOT }\end{array}$ & 83.699 & 9.09 & 1.9 & MOR & 718 & 44 & 185 & D D $\| \mathrm{D} \mathbf{0 . 2 6}$ & 1.52 & 4.93 & 5.08 & -132 \\
\hline $\begin{array}{c}00915153347.8234 .830 \\
\text { SC, OLD PICKENS }\end{array}$ & 82.971 & 2.45 & 2.2 & MOR & $14 \mid 19$ & 58 & 217 & $\mathrm{D} C \| \mathrm{D} \mathbf{0 . 4 2}$ & 1.45 & 3.07 & 6.56 & -105 \\
\hline $\begin{array}{c}0091641843.44 \quad 34.837 \\
\text { SC, OLD PICKENS }\end{array}$ & 82:899 & 7.58 & 2.5 & MOR & $5 \mid 8$ & 58 & 241 & D D ||D 0.34 & 2.37 & 5.91 & 16.52 & -131 \\
\hline $\begin{array}{c}0091620541.8934 .833 \\
\text { SC, OLD PICKENS }\end{array}$ & 82.979 & 6.03 & 1.9 & MOR & 517 & 58 & 220 & D D $\| \mathrm{D} \mathbf{0 . 3 6}$ & 2.68 & 4.80 & 22.34 & -124 \\
\hline $\begin{array}{c}9009174412.46 \quad 34.809 \\
\text { SC, OLD PICKENS }\end{array}$ & 82.954 & 6.15 & 2.5 & MOR & $6 \mid 9$ & 61 & 224 & D C $\| D \quad 0.25$ & 1.53 & 2.54 & 9.55 & -124 \\
\hline $\begin{array}{c}20091745344.2434 .803 \\
\text { SC, OLD PICKENS }\end{array}$ & 82.966 & 9.54 & 1.6 & MOR & $3 \mid 5$ & 61 & 278 & D D $\| \mathrm{D} 0.29$ & 3.98 & 14.43 & 99.00 & -116 \\
\hline $\begin{array}{c}0091752143.4234 .824 \\
\text { SC, WALHALLA }\end{array}$ & 83.000 & 5.91 & 1.7 & MOR & $3 \mid 6$ & 59 & 273 & D D $\| \mathrm{D} 1.36$ & 12.58 & 26.92 & 99.00 & -131 \\
\hline $\begin{array}{c}0091764038.56 \quad 34.801 \\
\text { SC, OLD PICKENS }\end{array}$ & 82.985 & 8.43 & 1.7 & MOR & 316 & 62 & 276 & $\mathrm{D} D \| \mathrm{D} \quad 0.53$ & 3.30 & 13.84 & 99.0 & 122. \\
\hline $\begin{array}{r}00918225550.2135 .340 \\
\text { NC, SANTEELAH CR }\end{array}$ & $\begin{array}{l}83.827 \\
\text { EEK }\end{array}$ & 6.56 & 1.1 & MOR & $5 \mid 7$ & 32 & 100 & $\mathrm{C} \mathrm{C} \| \mathrm{C}=.22$ & 1.77 & 3.84 & 99.00 & -23 \\
\hline $\begin{array}{c}00919,42010.10 \quad 34.815 \\
\text { SC, OLD PICKENS }\end{array}$ & 82.068 & $15: 30$ & 2.1 & MOR & $9 \mid 12$ & 60 & 220 & $\mathrm{DC} \| \mathrm{D} \quad 0.43$ & 1.08 & 6.03 & 99.00 & -112 \\
\hline $\begin{array}{r}0091951928.7134 .810 \\
\text { SC, OLD PICKENS }\end{array}$ & 82.963 & 5,80 & 2.1 & MOR & $12 \mid 17$ & 61 & 218 & D D $\| \mathrm{D} \quad 0.62$ & 1.48 & 3.09 & 8.00 & -114. \\
\hline $\begin{array}{r}9009195325.65 \quad 34.846 \\
\text { SC, OLD PICKENS }\end{array}$ & 82.997 & 14.86 & 0.0 & MOR & $5 \mid 7$ & 57 & 217 & D D $\| \mathrm{D} \mathbf{0 . 3 2}$ & 2.71 & 5.84 & 99.00 & -121 \\
\hline $\begin{array}{c}90091953656.6134 .838 \\
\text { SC, WALHALLA }\end{array}$ & 83.002 & 5.17 & 3.0 & MOR & $6 \mid 10$ & 58 & 218 & D C $\| \mathrm{D} 0.46$ & 1.64 & 6.15 & 9.79 & -129. \\
\hline $\begin{array}{c}90091954755.79 \quad 34.736 \\
\text { SC, SENECA }\end{array}$ & 82.834 & 3.95 & 2.0 & MOR & 5 & 69 & 234 & D D $\| D$ 1.0? & 2.23 & 8.68 & 15.59 & -127 \\
\hline $\begin{array}{c}90091955018.93 \quad 34.863 \\
\text { SC, OLD PICKENS }\end{array}$ & 82.898 & 15.65 & 2.1 & MOR & $5 \mid 9$ & 55 & 214 & D C $\| \mathrm{D} \quad 0.41$ & 1.45 & 5.70 & 89.00 & -113 \\
\hline $\begin{array}{c}90091965543.13 \quad 34.826 \\
\text { SC, OLD PICKENS }\end{array}$ & 82.973 & 7.58 & 2.2 & MOR & $11 \mid 17$ & 59 & 217 & D D $\| \mathrm{D} 0.62$ & 1,54 & 3.73 & 6.55 & -117. \\
\hline $\begin{array}{c}009198144.85 \quad 34.868 \\
\text { SC, WALHALLA }\end{array}$ & 83.016 & 16.19 & 2.7 & $\mathbf{R}$ & $4 \mid 7$ & 54 & 233 & 0.40 & 1.77 & 5.88 & 62.5 & 7. \\
\hline
\end{tabular}


DATE ORGIN LAT ${ }^{\circ} \mathrm{N}$ LONG ${ }^{\circ}$ W DEP MAG MOD ST|PH DMIN GAP Q SQD RMS ERH1 ERH2 ERZ AZM $00091982234.55 \quad 34.845$ SC, OLD PICKENS

$90091983012.84 \quad 34.860$ SC, WALHALLA

$90091983456.68 \quad 34.841$ SC, WALHALLA 900919215644.7634 .538 GA, DOUGHERTY 90092274716.1434 .861 SC, OLD PICKENS

$900922938 \cdot 48.28 \quad 34.885$ SC, SALEM

$90092295031.76 \quad 34.846$ SC, WALHALLA

90092210217.3434 .797 SC, OLD PICKENS

$90092334630.55 \quad 34.781$ SC, OLD PICKENS

$90092321618.0236 .420 \quad 82.504$
TN, SULLIVAN GARDENS 900924121323.8634 .766
SC, OLD PICKENS 90092815636.1735 .313 NC, HEWITT

$90100713220.23 \quad 36.079$ NC, NEWLAND

90100724332.5734 .798 SC, OLD PICKENS

$90101041520.93 \quad 35.006$ NC, CASHIERS 901011103847.2735 .849 TN, WALDEN CREEK

$0010232466.51 \quad 34.837$
SC, OLD PICKENS

$90110422227.46 \quad 35.390$ TN, MT VERNON

901107195537.9535 .103 NC, REID

$90110810210.93 \quad 36.954$ KY, LOUELLEN

901114153746.0534 .827 SC, OLD PICKENS

901118192925.7635 .613 TN, MADISONVILLE

$001125 \quad 55431.40 \quad 35.405$ NC, TUSKEEGEE

90120922115.7035 .545

$\begin{array}{lllllllllllll}82.978 & 16.29 & 1.5 & \text { MOR } & 4 \mid 6 & 66 & 229 & \text { D C } & 0.13 & 1.31 & 8.41 & 99.00 & -118\end{array}$

$\begin{array}{llllllllllllllll}83.639 & 6.16 & 1.9 & \text { MOR } & 8 \mid 10 & 7 & 73 & \text { C } & C \| B & 0.23 & 0.99 & 2.18 & 2.91 & -5 .\end{array}$

\begin{tabular}{lllllllll|llllll}
81.922 & 8.57 & 1.7 & MOR & $4 \mid$ & 6 & 11 & 249 & D D & 1 & 0.14 & 1.57 & 6.35 & 2.37 & 45
\end{tabular}

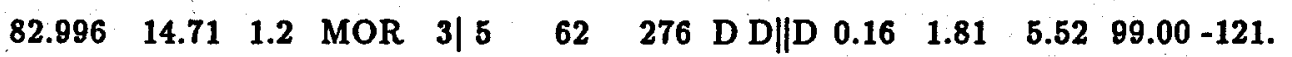

$\begin{array}{lllllllllllllll}83.008 & 6.03 & 0.9 & \text { MOPR } & 4 & 7 & 39 & 259 & \text { D D } & \text { D } & 0.29 & 2.26 & 6.00 & 18.91 & -128 .\end{array}$

$\begin{array}{llllllllllllllll}83.680 & 21.20 & 0.3 & \text { MOR } & 4 & 7 & 23 & 284 & \text { C } & \text { B } & \text { D } & 0.06 & 0.75 & 1.51 & 0.84 & -66 .\end{array}$

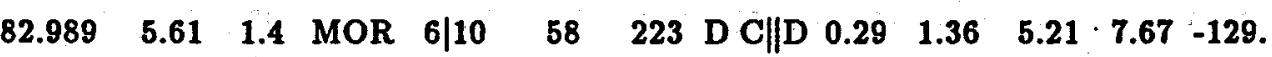

\begin{tabular}{lllll|llllllllll}
84.372 & 4.28 & 1.2 & MOR & 5 & 7 & 10 & 159 & $\mathbf{C}$ & $\mathrm{C} \| \mathrm{C}$ & 0.08 & 1.27 & 3.31 & 4.69 & -14.
\end{tabular}

$\begin{array}{llllllllllllllll}82.977 & 5.40 & 2.4 & \text { MOR } & 9 \mid 11 & 28 & 177 & \mathrm{C} & \mathrm{C} \| \mathrm{C} & 0.24 & 1.25 & 2.07 & 6.80 & -111\end{array}$

$\begin{array}{llllllllllllll}83.053 & 2.46 & 2.7 & \text { MOR } & 15 \mid 17 & 65 & 225 & \text { D C } & \text { D } & 0.19 & 1.34 & 3.59 & 5.18 & -105\end{array}$

$\begin{array}{llllllllllllll}82.988 & 5.69 & 1.9 & \text { MOR } & 9 \mid 12 & 59 & 219 & \text { D C } & \text { C } & 0.42 & 1.34 & 3.36 & 6.83 & -119\end{array}$

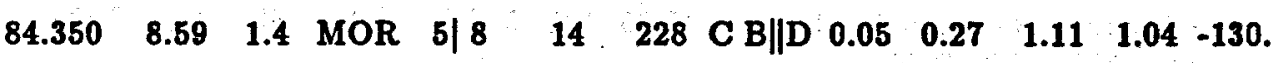

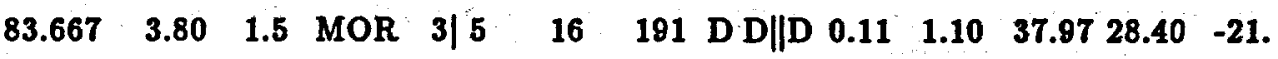

$\begin{array}{lllllllllllllll}84.430 & 11.15 & 1.3 & \text { MOR } & 4 \mid 6 & 24 & 231 & \text { C A } & \text { A D } & 0.03 & 0.26 & 1.11 & 0.79 & 33 .\end{array}$ TN, SWEETWATER 
DATE ORGIN LAT $^{\circ} \mathrm{N}^{2}$ LONG ${ }^{\circ} \mathrm{W}$ DEP MAG MOD ST|PH DMIN GAP Q SQD RMS ERH1 ERH2 ERZ AZM

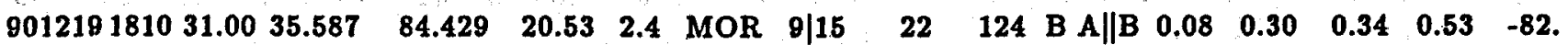
TN, SWEETWATER

\section{$9101145630.26 \quad 36.078 \quad 83$
TN, JEFFERSON CITY}

$91011561528.73 \quad 36.054 \quad 83.423$ TN, JEFFERSON CITY

\section{$91011521345.94 \quad 36.074 \quad 83.804$ TN, JOHN SEVIER}

\section{$910116182214.78 \quad 36.075 \quad 83.423$} TN, JEFFERSON CITY

\section{$910121 \quad 140 \quad 57.34 \quad 36.077 \quad 83.794$ TN, JOHN SEVIER}

\section{$91012614217.29 \quad 36.714 \quad 84.403$} KY, WHITLEY CITY

\section{$910127203541.68 \quad 36.362 \quad 82.529$} TN, LEESBURG

$\theta 10219 \quad 11515.37 \quad 35.498$ TN, MT VERNON

\section{$9102191401.36 \quad 35.613$ TN, VONORE}

\section{$91030734458.00 \quad 36.419$} TN, AUSMUS

$91030793140.49 \quad 35.482$ TN, RAFTER

$910415 \quad 555 \quad 46.73 \quad 35.591$ NC, SILERS BALD

910428193743.8935 .730 TN, BINFIELD

$9105053216.79 \quad 36.336$ TN, WHITE HOLLOW

$910510135042.98 \quad 35.757$ TN, ROCKWOOD 910510194036.5834 .865
GA, NICKAJACK GAP

$91051914429.10 \quad 35.312$ TN, CHARLESTON

$91052010524.08 \quad 34.742$ GA, VILLANOW

$910526 \quad 54927.26 \quad 35.488$ TN, RICEVILLE

$91052823449.02 \quad 35.310$ NC, HEWITT

$\begin{array}{lll}910529 & 247 \quad 18.86 \quad 35.415\end{array}$ TN, MT VERNON

91061361452.8434 .965 GA, BEAVERDALE

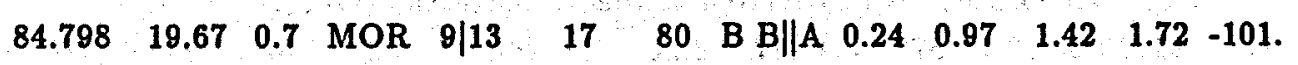

$\begin{array}{llllllllllllllllll}85.112 & 6.84 & 0.9 & \text { MOR } & 7 \mid 11 & 34 & 177 & \text { C C } \| C & 0.19 & 0.63 & 1.47 & 14.46 & -118\end{array}$

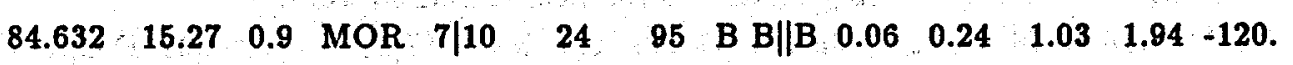

$\begin{array}{lllllllllllllll}83.628 & 2.63 & 2.2 & \text { MOR } & 15 \mid 19 & 41 & 114 & \text { C C } & \| C & 0.40 & 1.19 & 1.77 & 3.35 & 28\end{array}$

$\begin{array}{lllllllllllllllll}84.292 & 21.59 & 2.9 & \text { MOR } 19 \mid 31 & 18 & 66 & \text { B } & \text { B } & 0.19 & 0.19 & 0.36 & 0.65 & 0.80 & -127\end{array}$

$\begin{array}{lllllllllllllllll}84.808 & 14.69 & 1.4 & \text { MOR } & 6 \mid 10 & 17 & 263 & \text { D C }|| D & 0.15 & 1.46 & 3.79 & 2.24 & -27\end{array}$ 
DATE ORGIN LAT ${ }^{\circ} \mathrm{N}^{2} \mathrm{LNG}^{\circ} \mathrm{W}$ DEP MAG MOD ST|PH DMIN GAP Q SQD RMS ERH1 ERH2 ERZ AZM $\begin{array}{lllllllllllllllll}910613 & 2246 & 2.76 & 35.569 & 84.734 & 13.62 & 1.0 & \text { MOR } & 11 \mid 18 & 36 & 80 & \text { B A } & 0.12 & 0.32 & 0.35 & 0.54 & -54\end{array}$ TN, TRANQUILLITY

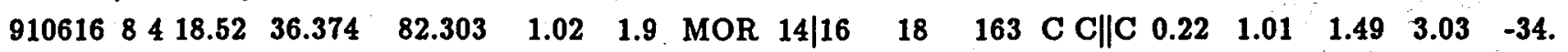
TN, JOHNSON CITY

$\begin{array}{llllllllllllllllllllll}910620 & 720 & 48.77 & 35.756 & 84.202 & 23.16 & -0.1 & \text { MOR } & 8 & 13 & 10 & 97 & \text { C C } & 0.31 & 1.03 & 1.83 & 2.29 & 38 .\end{array}$ TN, CONCORD

$\begin{array}{lllllllllllllllllll}910623 & 1854 & 5.64 & 36.015 & 83.816 & 11.88 & 0.9 & \text { MOR } & 3 \mid 6 & 40 & 312 & \text { D } & \text { D }|| D & 0.21 & 3.55 & 6.40 & 5.85 & -78 .\end{array}$ TN, JOHN SEVIER

\begin{tabular}{lllllll|lllllllllll}
910711 & 026 & 15.70 & 36.052 & 83.816 & 8.72 & 0.9 & MOR & 4 & 5 & 44 & 360 & D D ||D & 0.06 & 3.49 & 7.12 & 4.89 & -81.
\end{tabular} TN, JOHN SEVIER

$91071473156.02 \quad 35.562$ TN, SWEETWATER

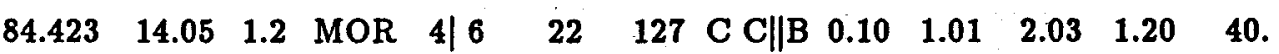

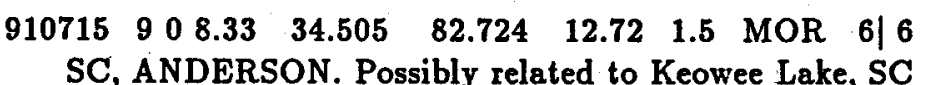

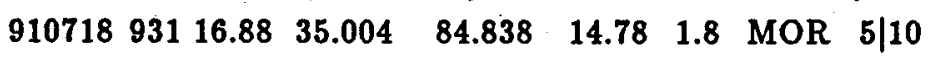
TN, FELKER

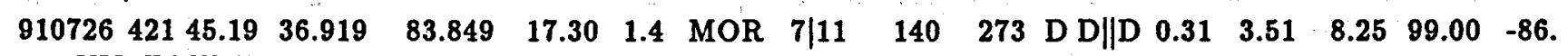
KY, FOUNT

$\begin{array}{llllllllllllllllll}910729 & 1533 & 21.59 & 35.133 & 84.820 & 13.53 & 1.7 & \text { MOR } & 7 \mid 13 & 33 & 247 & \text { C B } & \text { B } & 0.16 & 0.64 & 1.31 & 0.83 & 12 .\end{array}$ TN, EAST CLEVELAND

$\begin{array}{lllllllllllllllll}910731 & 744 & 14.11 & 35.014 & 84.360 & 11.44 & 0.4 & \text { MOR } & 5 / 8 & 33 & 132 & \text { C C }|| C & 0.19 & 1.11 & 2.33 & 2.12 & -37 .\end{array}$ TN, ISABELLA

$\begin{array}{llllllllllllllllll}910817 & 1759 & 9.82 & 34.924 & 85.451 & 19.92 & 2.7 & \text { MOR } & 9 \mid 13 & 11 & 318 & \text { C B } & \text { B } & 0.12 & 1.04 & 1.56 & 1.12 & -35 .\end{array}$ GA, HOOKER

$\begin{array}{llllllllllllllllllllll}910818 & 1532 & 16.84 & 35.473 & 84.072 & 14.89 & 0.4 & \text { MOR } & 4 & 7 & 25 & 192 & \text { C B |D } & 0.09 & 0.28 & 1.02 & 2.51 & -111 .\end{array}$ TN, WHITEOAK FLATS

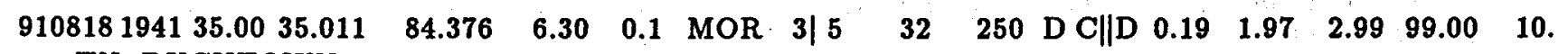
TN, DUCKTOWN

$\begin{array}{llllllllllllllll}9109082214 & 19.92 & 36.154 & 83.793 & 5.93 & 1.8 & \text { MOR } & 7 \mid 11 & 51 & 253 & \text { D C }|| D & 0.21 & 0.62 & 2.23 & 2.97 & 13 .\end{array}$ TN, GRAVESTON

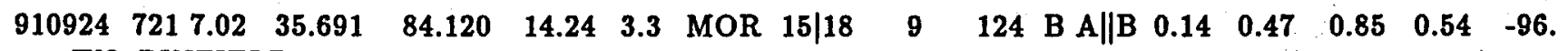
TN, BINFIELD

$\begin{array}{lllllllllllllll}911007 & 12 & 4 & 6.26 & 35.856 & 82.026 & 32.79 & 2.6 & \text { MOR } & 4 \mid 7 & 35 & 202 & D & D & 0\end{array}$ NC, LITTLE SWITZERLAND

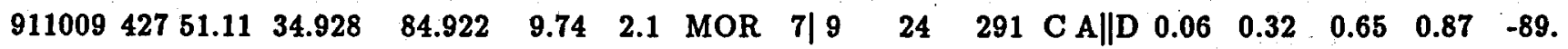
GA, COHUTTA. Possibly related to road construction(GIT).

$\begin{array}{llllllllllllllllllll}911014 & 1110 & 37.62 & 35.578 & 84.187 & 8.20 & 1.9 & \text { MOR } & 5 / \theta & 10 & 126 & \text { B } & \text { B } \mid \text { B } & 0.12 & 0.52 & 1.38 & 2.31 & -115 .\end{array}$ TN, VONORE

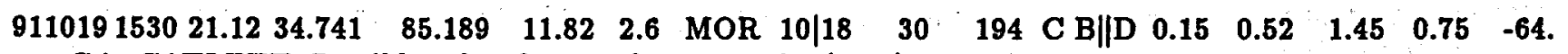
GA, CATLETT. Possibly related to road construction(GIT).

$\begin{array}{lllllllll}911021 & 110 & 48.78 & 34.735 & 85.151 & 14.43 & 2.0 & \text { MOR } & 7 \mid 11\end{array}$ GA, CATLETT

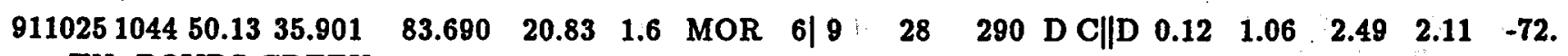
TN, BOYDS CREEK

$\begin{array}{llllllllllllllllll}911027 & 141 & 5.53 & 35.007 & 84.381 & 12.14 & 0.6 & \text { MOR } & 4 / 7 & 31 & 243 & \text { C B } & \text { B|D } & 0.15 & 1.01 & 1.67 & 2.38 & -44 .\end{array}$ TN, DUCKTOWN

$\begin{array}{llllllllllllllllll}011028 & 1046 \cdot 20.69 & 35.611 & 84.721 & 13.06 & 1.6 & \text { MOR } & 7 \mid 12 & 33 & 180 & \text { B A AC } & 0.08 & 0.30 & 0.48 & 0.53 & -57 .\end{array}$ TN, TRANQUILLITY 
DATE ORGIN LAT $^{\circ} \mathrm{N}$ LONG ${ }^{\circ} \mathrm{W}$ DEP MAG MOD ST|PH DMIN GAP Q SQD RMS ERH1 ERH2 ERZ AZM

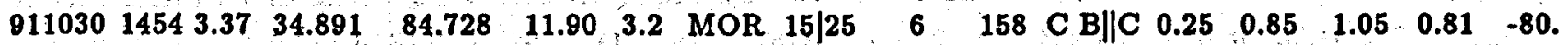
GA, TENNGA

$\begin{array}{llllllllllllllllllllllllll}911103 & 653 & 52.71 & 35.437 & 84.207 & 0.64 & 1.2 & \text { MOR } & 4 \mid 7 & 25 & 118 & \mathrm{C} & \mathbf{C} \| \mathrm{C} & 0.08 & 0.44 & 1.21 & 3.86 & 35 .\end{array}$ TN, RAFTER

$\begin{array}{llllllllllllllllllllll}911103 & 7 & 5 & 59.93 & 35.445 & 84.227 & 6.57 & 1.0 & \text { MOR } & 3 \mid & 6 & 25 & 178 & \mathrm{C} & \mathrm{C} \| \mathrm{C} & 0.05 & 0.45 & 1.92 & 17.22 & 38 .\end{array}$ TN, RAFTER

$\begin{array}{lllllllllllllllllll}911110 & 954 & 36.78 & 34.557 & 85.673 & 3.91 & 1.8 & \text { MOR } & 6 \mid & 7 & 55 & 315 & \text { C B } & 0.03 & 0.69 & 1.80 & 2.35 & -18\end{array}$ AL, DUGOUT VALLEY

$\begin{array}{llllllllllllllllllll}011111 & 23 & 6 & 4.49 & 35.557 & 84.470 & 13.08 & 1.3 & \text { MOR } & 6 \mid & 7 & 26 & 130 & \text { C C } \mid \text { B } & 0.20 & 1.61 & 2.37 & 2.84 & -38 .\end{array}$ TN, SWEETWATER

$91111585213.20 \quad 35.341$ TN, BIG JUNCTION

\begin{tabular}{lllll|llllllllll}
84.104 & 5.85 & 1.7 & MOR & 6 & 9 & 32 & 78 & C C & |C & 0.12 & 0.63 & 0.84 & 57.87 & -124.
\end{tabular}

$\begin{array}{lllllllll}9111172111 & 31.71 & 34.987 & 82.926 & 8.23 & 2.9 & \text { MOR } & 9 \mid 9 & 41\end{array}$ SC, SALEM. Possibly related to Jocassee Lake, SC.

$\begin{array}{llllllllll}911121 & 512 & 2.00 & 35.468 & 84.343 & 9.12 & 2.2 & \text { MOR } & 10 \mid 13 & 10\end{array}$ TN, MT VERNON

$\begin{array}{lllllllllll}911122 & 533 & 41.60 & 35.476 & 84.286 & 10.17 & 2.1 & \text { MOR } & 6 / 6 & 22\end{array}$ TN, MT VERNON

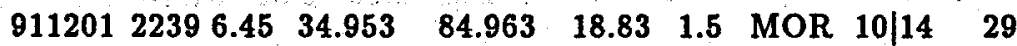
GA, COHUTTA. Possibly related to road construction(GIT).

$\begin{array}{lllllllllll}9112031515 & 20.7935 .580 & 84.111 & 8.31 & 1.2 & \text { MOR } & 5 \mid 8 & 13\end{array}$ TN, TALLASSEE

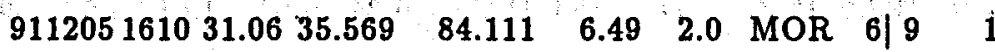
TN, TALLASSEE

91120643932.4535 .48 NC, WAYNESVILLE

$\begin{array}{lllllllll}911214 & 215 & 50.57 & 35.748 & 84.240 & 15.15 & 0.6 & \text { MOR } & 4 \mid 6 \quad 10\end{array}$ TN, MEADOW

$\begin{array}{llllllllllll}911222 & 256 & 10.57 & 35.378 & 84.795 & 9.86 & 1.9 & \text { MOR } & 5 / 6\end{array}$ TN, GOODFIELD

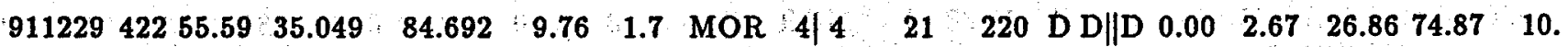
TN, PARKSVILLE

$92010345954.67 \quad 36.779 \quad 83.040 \quad 9.71 \quad 2.4 \quad$ MOR $13 \mid 15$ VA, PENNINGTON GAP

$9201042122.21 \quad 36.277 \quad 83.521 \quad 5.74 \quad 2.0$ MOR $16 / 22 \quad 33$ TN, DUTCH VALLEY

$\begin{array}{lllllllll}920214 & 1036 & 3.50 & 35.278 & 84.621 & 16.62 & 1.5 & \text { MOR } & 8 \mid 11\end{array}$ TN, ETOWAH

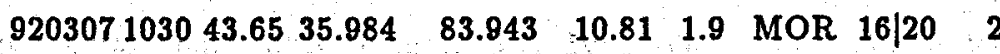
TN, KNOXVILLE

920312184158.09
NC, HEWITT

$\begin{array}{llllllll}9203132249 & 41.09 & 35.528 & 84.397 & 14.72 & 2.2 & \text { MOR } & 21 / 36 \quad 23\end{array}$ TN, SWEETWATER

$\begin{array}{lllllllllll}920317 & 847 & 25.63 & 35.387 & 84.992 & 3.07 & -0.5 & \text { MOR } & 8 & 13 & 28\end{array}$ TN, BIG SPRING

$\begin{array}{lllllllllll}920318 & 145 & 40.53 & 35.871 & 83.663 & 18.49 & 0.8 & \text { MOR } & 8 / 13 & 26\end{array}$ TN, WALDEN CREEK

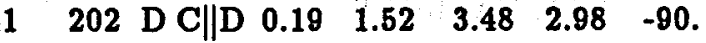

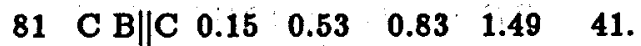

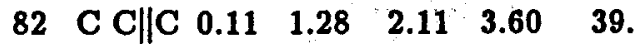

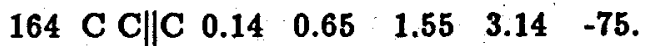
$\begin{array}{lllllllllll}117 & C & C & \| B & 0.09 & 0.55 & 1.33 & 2.75 & -107 .\end{array}$

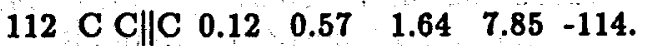
$\begin{array}{lllllllll}88 & \text { В }\end{array}$ $\begin{array}{llllllll}260 & D & D\end{array} \mid \mathrm{D} \quad 0.08 \quad 0.96 \quad 4.21 \quad 1.80 \quad-117$. $\begin{array}{lllllllll}250 & D & C & \| D & 0.10 & 1.70 & 2.74 & 3.38 & 36 .\end{array}$

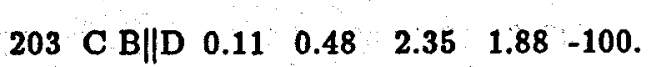
$\begin{array}{llllllll}89 & \mathrm{C} \cdot \mathrm{B} \| \mathrm{C} & 0.15 & 0.40 & 0.49 & 1.52 & 2 .\end{array}$ $\begin{array}{llllllllll}143 & \mathrm{C} & \mathrm{B} \| \mathrm{C} & 0.09 & 0.40 & 0.67 & 1.37 & -109\end{array}$ $\begin{array}{lllllllll}100 & C & B & \| C & 0.22 & 0.50 & 0.87 & 1.00 & -132\end{array}$

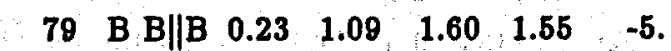
$\begin{array}{llllllllllll}50 & \mathrm{C} & \mathrm{C} \| \mathrm{B} & 0.31 & 0.56 & 0.67 & 1.86 & -110\end{array}$

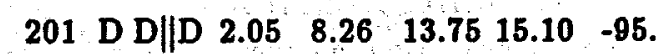

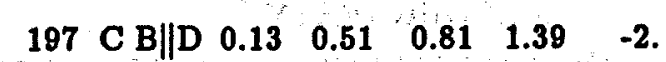


DATE ORGIN LAT $^{\circ} \mathrm{N}$ LONG ${ }^{\circ} \mathrm{W}$ DEP MAG MOD ST|PH DMIN GAP Q SQD RMS ERH1 ERH2 ERZ AZM

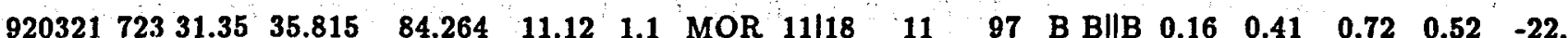
TN, LENOIR CITY

$920323 \quad 84736.35 \quad 35.932 \quad 84.021$ TN, BEARDEN

$92033092030.24 \quad 35.516 \quad 85.082$ TN, MORGAN SPRINGS

$920402111710.3535 .366 \quad 84.660$ TN, CALHOUN

$92042520159.1034 .904 \quad 84.686$

GA, TENNĢA

$\begin{array}{lllll}920426 & 417 & 1.79 & 34.719 & 85.132\end{array}$ GA, CATLETT

$\begin{array}{lllll}920505 \quad 129 & 56.15 \quad 35.247 \quad 84.527\end{array}$ TN, OSWALD DOME

$\begin{array}{llllll}920531 & 436 & 2.14 & 36.186 & 82.884\end{array}$ TN, MOSHEIM

$920607955 \quad 54.88 \quad 35.12$ NC, WAYAH BALD

$920614112739.7336 .135,83.949$ TN, BIG RIDGE PARK

$92062852145.76 \quad 36.671 \quad 83.636$
KY, MIDDLESBORO NORTH

$920628142910.28 \quad 34.742 \quad 85.269$ GA, ESTELLE

$\begin{array}{llllllllll}920702 & 347 & 42.71 & 34.870 & 86.349 & 7.68 & 1.9 & \text { MOR } & 15 \mid 22 & 30\end{array}$ AL, HOLLYTREE

920704162318.9136 .160

TN, LUTTRELL

$\begin{array}{lllllllllllllllllllllll}920706 & 10 & 9 & 41.79 & 35.505 & 84.244 & 13.88 & 0.4 & \text { MOR } & 4 & 5 & 28 & 360 & \text { D } & D \| D & 0.10 & 1.88 & 10.54 & 4.85 & -122 .\end{array}$ TN, VONORE

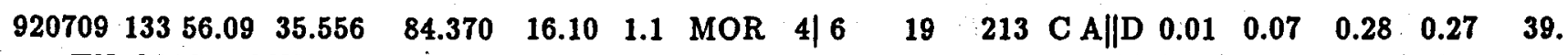
TN, MADISONVILLE

$\begin{array}{llllllllll}920716 & 058 & 3.51 & 34.876 & 84.915 & 24.22 & 1.3 & \text { MOR } & 5 & 7\end{array}$ GA, COHUTTA

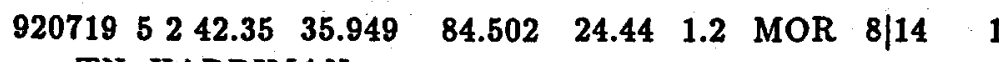
TN, HARRIMAN

$\begin{array}{llllllllll}920801 & 919 & 45.45 & 35.372 & 85.212 & 12.57 & 0.3 & \text { MOR } & 3 / 5 & 58\end{array}$ TN, SODDY

$\begin{array}{llllllllllllllllll}920803 & 1134 & 29.07 & 34.807 & 85.048 & 29.14 & 1.4 & \text { MOR } & 4 \mid 7 & 35 & 319 & \text { D C } & \text { C|D } & 0.15 & 2.96 & 6.54 & 5.34 & 16 .\end{array}$ GA, TUNNEL HILL

$\begin{array}{llllllllllllllllll}920805 & 342 & 50.75 & 35.685 & 84.396 & 16.77 & 0.2 & \text { MOR } & 3 \mid 5 & 17 & 270 & \text { D D } & 5 & 0.05 & 2.19 & 6.97 & 3.59 & -130 .\end{array}$ TN, PHILADELPHIA

$\begin{array}{llllllllllllllllllll}920808 & 351 & 41.80 & 34.576 & 85.431 & 8.54 & 1.9 & \text { MOR } & 6 \mid 10 & 45 & 304 & \text { C B } \mid \text { D } & 0.10 & 0.73 & 1.24 & 1.09 & -51 .\end{array}$ GA, DOUGHERTY

$\begin{array}{llllllllllllllllllllll}920812 & 416 & 53.54 & 35.292 & 84.301 & 22.57 & 0.9 & \text { MOR } & 10 \mid 16 & 15 & 86 & \text { A A } & \text { A A } & 0.10 & 0.32 & 0.41 & 0.66 & -133 .\end{array}$ TN, TELLICO PLAINS 
DATE ORGIN LAT ${ }^{\circ} \mathrm{N}$ LONG ${ }^{\circ} \mathrm{W}$ DEP MAG MOD ST|PH DMIN GAP $Q$ SQD RMS ERH1 ERH2 ERZ AZM

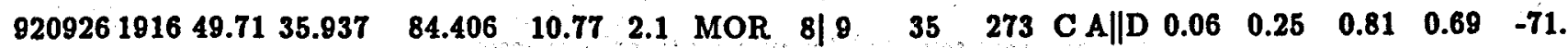
TN, ELVERTON

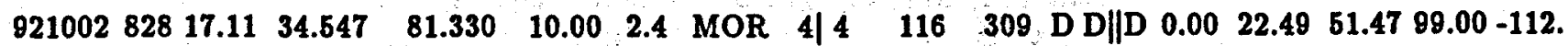
SC, CARLISLE SE

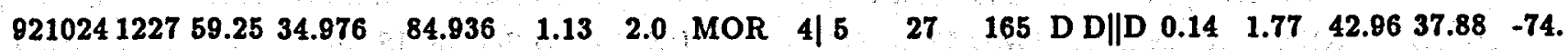
GA, COHUTTA

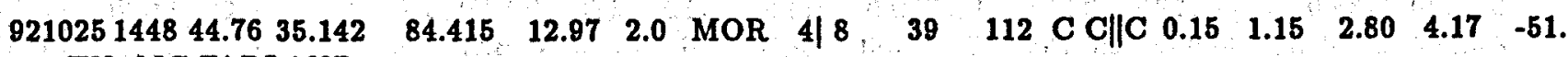
TN, MC FARLAND

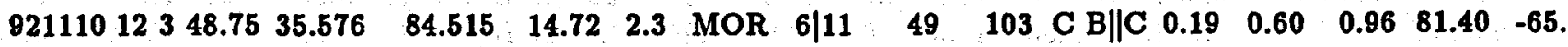
TN, NIOTA

$\begin{array}{lllllllllllllllllll}921110 & 1716 & 46.76 & 35.657 & 84.151 & 11.49 & 2.9 & \text { MOR } & 10 \mid 14 & 34 & 96 & \text { C B } & \text { CC } & 0.19 & 0.83 & 1.11 & 1.71 & -115 .\end{array}$ TN, MEADOW 


\section{(B) REGIONAL EARTHQUAKES (OUTSIDE SARSN)}

Hypocenters reported for informational purposes only consult VPI, GIT, USC/USGS for more complete data

All events from September 1981 - December 1985 were located using HYPO71.

DATE ORGIN LAT ${ }^{\circ}$ LONG $^{\circ}$ W DEP MAG ST|PH DMIN GAP Q SQD RMS ERH1 ERH2 ERZ AZM

\begin{tabular}{|c|c|c|c|c|c|c|c|c|c|c|c|c|c|c|c|}
\hline & 0.100 & .1289 & & 1.0 & & 618 & 12 & 31 & D & D & 0.20 & & & & \\
\hline & 6.51 & & & 7.1 & 0.0 & $15 \mid 20$ & 6 & 127 & B & $\mathrm{B} \mid \mathrm{B}$ & 0.30 & 1.10 & .00 & & \\
\hline & 3295.68 & & & 10.1 & 0.0 & $7 \mid 11$ & 1 & 95 & B & $\mathbf{A} \mid \mathbf{B}$ & 0.11 & & & & \\
\hline & 4521.43 & 2.914 & & 11.0 & 0.0 & $6 \mid 9$ & 124 & 11 & 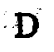 & $\mathrm{D} \mid \mathrm{D}$ & 0.32 & & & & \\
\hline & 9.67 & 2.827 & 89 & 15.8 & .0 & $7 \mid 14$ & 59 & 24 & & D|D & 3.65 & .20 & 00 & & \\
\hline & 5.04 & 2.689 & 6.646 & 25.2 & 0.0 & $6 \mid 12$ & 74 & 279 & & D|D & 1.04 & & 0 & & \\
\hline 01 & 9.76 & 33.6 & & 1.0 & 0.0 & & 68 & 78 & & & 0.14 & & & & \\
\hline 0413 & 9.30 & 34.2 & 60 & 12.0 & 3.1 & 6| 8 & 3 & 57 & & & 0.22 & 7.10 & & & \\
\hline 0512 & 3.88 & 34.2 & 7.528 & 7.9 & 2.4 & $8 \mid 13$ & 95 & 11 & & $\mathbf{D}$ & 0.25 & 2.40 & .00 & 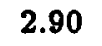 & \\
\hline 0 & 8.98 & 9 & 26 & 20.0 & 0.0 & $4 \mid 8$ & 73 & 80 & & D & 0.25 & 1.70 & .00 & 90 & \\
\hline & 4.01 & 37.882 & 75 & 11.1 & 0.0 & $8 \mid 15$ & 34 & 243 & & $\mathbf{B} \mid \mathbf{D}$ & 0.19 & & & & \\
\hline 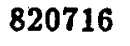 & 0.66 & 34.201 & 1.429 & 6.7 & .9 & $5 \mid 10$ & 188 & 298 & & $\mathbf{B} \mid \mathbf{D}$ & 0.19 & 2. & .00 & & \\
\hline 208 & .33 & 34.306 & 10 & 44.7 & 0.0 & $4 \mid 8$ & 127 & 277 & & D & 3.13 & 0 & 0 & & \\
\hline & 30 & 32.5 & 16 & 0.6 & 3. & $14 \mid 19$ & 149 & 222 & & & 0.31 & & & & \\
\hline 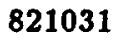 & .00 & 32.6 & 2 & 0.3 & .1 & $14 \mid 17$ & 255 & 59 & & $\mid D$ & 1.89 & 60 & .00 & & \\
\hline 8 & 1.34 & 32.644 & 54 & 2.4 & 0.0 & & 175 & 319 & & & 3.40 & & .00 & & \\
\hline 1 & 79 & 32.8 & 2 & 11.0 & 0.0 & $13 \mid 23$ & 152 & 275 & 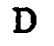 & & & & 0 & & \\
\hline & 39 & 4 & 1 & 0.6 & 0.0 & 81 & 153 & 46 & 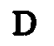 & D & 32 & & 0 & & \\
\hline 17 & .54 & $A$ & 3 & 15.8 & 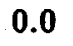 & & 180 & 253 & 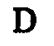 & & 2.53 & & 00 & & \\
\hline s & 7.29 & 37.3 & 7 & 22.0 & 2.4 & 315 & 105 & 42 & 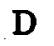 & & 0.11 & 4.70 & 0.00 & & \\
\hline & .52 & 3 & 5 & 17.0 & 2 & $6 / 9$ & 0 & 170 & 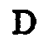 & & 12.56 & & & & \\
\hline & & & & & & & 30 & 166 & & & & & & & \\
\hline 10 & 11 & 37 & 3.044 & 17.9 & 5 & 8114 & 73 & 202 & $\mathbf{n}$ & C|D & 0.27 & 2 & U & 0 & \\
\hline & .25 & 4 & 10 & 15.0 & .3 & $4 \mid 8$ & 238 & 289 & D & D & 0.12 & 5. & U. & 6.30 & \\
\hline & 3 & & & 1.0 & 0 & $7 \mid 10$ & 150 & 281 & 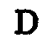 & D & 0.13 & 0 & & & \\
\hline 6 & & & & 11.0 & & & & 9 & C & & & & 0 & & \\
\hline 20 & 7.00 & 38.0 & 6 & 0.7 & & & 16 & 40 & $\mathrm{D}$ & $\mathrm{D} \mid \mathrm{D}$ & 0.71 & 50 & 0.00 & 17.70 & \\
\hline 7 & 7.19 & 38.307 & 10 & 20.0 & .4 & 9 & 80 & 242 & $\mathbf{D}$ & $\mathrm{D} \mid \mathrm{D}$ & 1.11 & 10 & 0 & 4.40 & \\
\hline & & & & 13.1 & $a_{2}$ & & 81 & 302 & D & |D & 0.31 & & 0 & & \\
\hline & & & & 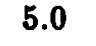 & & & 0 & 31 & D & & & & 0 & & \\
\hline & 4.73 & 32. & & 40.5 & 2.5 & & 41 & 171 & $\mathbf{D}$ & 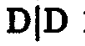 & $\mathbf{0}$ & 56 & 0.00 & 43.90 & \\
\hline & 1.31 & 37.625 & & 0.3 & 10 & t & 163 & 283 & D & C|D & 0.29 & 0 & 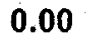 & 7.80 & \\
\hline & & & & 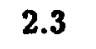 & 2 & & y & 324 & D & & 0.33 & & 0 & & \\
\hline & & & & 6.1 & & & & 344 & $\mathbf{n}$ & & & & & & \\
\hline 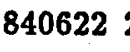 & .69 & 37 & & 5.0 & & $4 \mid 6$ & 106 & 276 & D & $\mathbf{D}$ & 4.92 & & 0.00 & & \\
\hline nt & 7.89 & 37.2 & 8 & 7.3 & 2.0 & $6 \mid 8$ & 17 & 136 & C & C & 0.12 & 0.80 & 0 & 0 & \\
\hline & 20 & & & 12.7 & 1.9 & & 25 & 166 & B & & 0.14 & & & & \\
\hline & & & & 2 & 1.4 & & 12 & 3 & 0 & & 1 & & & & \\
\hline & 20 & & & 0.2 & & & 179 & 257 & D & D & 0.29 & & & & \\
\hline 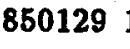 & 58.24 & 33.980 & & 2.3 & & $15 \mid 26$ & 25 & 276 & $\mathbf{c}$ & D & 0.24 & & & 1.50 & \\
\hline & 19.52 & 33.688 & & 5.0 & & & 73 & 271 & D & & 0.08 & & 0.00 & 5.50 & 0. \\
\hline & 38.55 & 37.2 & 80.515 & 4.6 & 2.9 & & 11 & 107 & B & & 0.13 & 0.70 & 0.00 & 0.90 & 0. \\
\hline & & 33.766 & & -0 & & & 129 & 223 & D & D & 0.44 & & & & \\
\hline & & 34.434 & 82.754 & 8.2 & 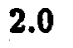 & $12 \mid 18$ & 32 & 154 & 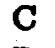 & & 0.36 & 2.00 & 0.00 & 4.50 & . \\
\hline & & & & 0.5 & 4 & & 26 & 180 & $\mathbf{D}$ & & 0.11 & 1.27 & 2.33 & 0.90 & \\
\hline I. & 22929.65 & .098 & 3.230 & 2.4 & 5 & $16 \mid 7$ & 204 & 15 & $\boldsymbol{D}$ & D & 0.08 & 2.23 & 4.81 & 1.85 & -00 \\
\hline
\end{tabular}


DATE ORGIN LAT $^{\circ} \mathrm{N}^{2}$ LONG $^{\circ}$ W DEP MAG ST|PH DMIN GAP Q SQD RMS ERH1 ERH2 ERZ AZM

\begin{tabular}{|c|c|c|c|c|c|c|c|c|c|c|c|c|c|}
\hline 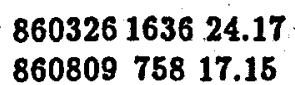 & $\begin{array}{l}37.252 \\
36.793\end{array}$ & $\begin{array}{l}80.516 \\
80.740\end{array}$ & $\begin{array}{l}7.0 \\
12.3\end{array}$ & $\begin{array}{l}3.0 \\
2.4\end{array}$ & $\begin{array}{l}41 \mid 26 \\
12 \mid 20\end{array}$ & $\begin{array}{c}9 \\
27\end{array}$ & $\begin{array}{l}490 \\
800\end{array}$ & $\begin{array}{l}\text { B A } \mid C \\
\text { C A } \mid D\end{array}$ & $\begin{array}{l}0.10 \\
0.13\end{array}$ & $\begin{array}{l}0.30 \\
0.39\end{array}$ & $\begin{array}{l}0.36 \\
0.63\end{array}$ & $\begin{array}{l}0.20 \\
0.50\end{array}$ & $\begin{array}{r}-99.0 \\
27.0\end{array}$ \\
\hline 86082974438.20 & 34.290 & 85.484 & 18.5 & 1.4 & $7 / 9$ & 10 & 40 & $\mathrm{D}, \mathrm{D} \mid \mathrm{D}$ & 0.19 & 1.09 & 4.70 & 2.40 & 119.0 \\
\hline $860829 \quad 749 \quad 3.49$ & 34.389 & 85.538 & 10.3 & 1.5 & $15 \mid 22$ & 22 & 500 & B A $\mid \mathrm{C}$ & 0.15 & 0.42 & 0.54 & 0.40 & -106.0 \\
\hline 61003102149.45 & 35.805 & 80.456 & 0.2 & 2.5 & $12 \mid 17$ & 69 & 600 & C|D & 0.25 & 1.13 & 3.14 & 3.50 & 8.0 \\
\hline 101722 & 35.546 & 86.041 & 7.8 & 2.3 & $11 \mid 18$ & 2 & 440 & $3 \mid \mathrm{C}$ & 0.20 & 0.69 & 1.12 & 0.50 & -129.0 \\
\hline 200 & 33.193 & 83.229 & 11.7 & 2.4 & $9 \mid 8$ & 107 & 560 & $\mathrm{D} \mid \mathrm{D}$ & 0.15 & 1.46 & 5.90 & 3.80 & -128.0 \\
\hline $861220 \div 8132.89$ & 38.036 & 80.619 & 14.8 & 1.9 & $6 \mid 9$ & 53 & 260 & D & 0.22 & 0.82 & 5.91 & 22.20 & 43.0 \\
\hline
\end{tabular}

DATE ORGIN LAT ${ }^{\circ} \mathrm{NLONG}^{\circ} \mathrm{W}$ DEP MAG MOD ST|PH DMIN GAP Q SQD RMS ERH1 ERH2 ERZ AZM

$8701064539.56 \quad 33.158$

870113111138.8238 .340

$87012710100.65 \quad 35.822$

$87012873012.15 \quad 33.181$

$87012984018.75 \quad 33.254$

870201161235.6933 .226

870202121312.1933 .162

870214122318.1732 .709

$87021944320.74 \quad 33.219$

$87022452533.91 \quad 33.228$

$8703107526.66 \quad 34.551$

$87031613926.38 \quad 34.556$

870510194741.8937 .793

870629104921.4234 .844

$\begin{array}{lllll}870708 & 145 & 0.61 & 34.832\end{array}$

$870709: 35143.00 \quad 33.249$

871018161634.4734 .191

$871121 \quad 1321.24 \quad 36.773$

$871128 \quad 85039.53 \quad 33.968$

$87121235328.10 \quad 34.154$

$871212 \quad 438 \quad 57.89 \quad 34.159$

87121210325.7434 .163

$871224224644.22 \quad 34.164$

88010713224.6833 .967

$880126 \quad 146 \quad 25.28 \quad 34.195$

88012722542.8534 .189
83.169

83.415

80.466

83.152

83.181

83.270

83.166

87.269

83.159

83.213

80.946

80.961

83.393

87.658

87.612

83.307

87.066

80.751

82.533

82.714

82.668

82.673

82.723

82.641

82.738

82.750
19.061 .7 MOR $6 \mid 10$

25.832 .3 MOR $15 \mid 23$

5.00R 2.1 MOR 14|24

11.601 .7 MOR $6 / 10$

5.00R 2.5 MOR 10|17

2.131 .5 MOR 9|15

1.57 1.7 MOR 7/8

7.292 .8 MOR 16|23

16.952 .1 MOR $5 / 7$

0.60 1.8 MOR 5 | 9

8.81 1.8 MOR 12|23

9.99 3.2 MOR 16|26

$0.68 \quad 3.0$ MOR $17 \mid 25$

12.72 2.2 MOR $13 \mid 19$

$\begin{array}{llll}8.56 & 2.1 & \text { MOR } & 12 \mid 19\end{array}$

8.152 .0 MOR $7 / 9$

2.512 .1 MOR 16/26

12.122 .5 MOR $14 \mid 26$

13.74 1.5 MOR 12/21

$\begin{array}{lll}9.88 & 3.3 \text { MOR 20|28 }\end{array}$

$\begin{array}{lllll}9.06 & 1.4 & \text { MOR } & 7 / 9\end{array}$

5.021 .5 MOR 12|19

$\begin{array}{llll}6.70 & 3.0 & \text { MOR } 23 / 36\end{array}$

14.71 1.6 MOR 8|14

1.892 .6 MOR 16|29

6.06 1.9 MOR 10|19

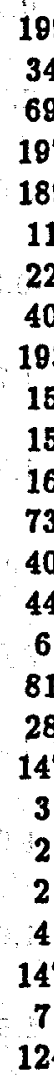

$304 \mathrm{C} B \| \mathrm{D} 0.08 \quad 1.05$

$\begin{array}{lll}191 & D\end{array}$

260 D D |D $0.52 \cdots 1.51$

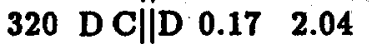

302 D D ||D $0.48 \quad 3.80$

254 D C ||D $0.41 \quad 1.80$

360 D D |D 0.432 .62

$\begin{array}{llll}225 & D & C\end{array} \mid D \quad 0.30 \quad 0.67$

$325 \mathrm{D} C|| \mathrm{D} \quad 0.12 \quad 1.88$

$\begin{array}{llll}277 & D\end{array}$ C $\mid$ D $0.20 \quad 2.28$

$\begin{array}{llll}127 & \text { C C ||B } & 0.20 & 0.53\end{array}$

$\begin{array}{lllll}126 & \mathrm{C} \mathrm{C} \| \mathrm{B} & 0.23 & 0.61\end{array}$

100 C B |D $0.21 \quad 0.63$

196 C B

$\begin{array}{lll}116 & C \\ B \| C & 0.20 & 0.72\end{array}$

311 C B |D $0.05 \quad 0.75$

243 C B ||D $0.21 \quad 0.61$

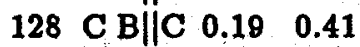

273 D D |D 0.602 .52

$\begin{array}{lllll}101 & \text { B B ||B } 0.16 \quad 0.38\end{array}$

$\begin{array}{llllll}281 & \mathrm{D} C & \mathrm{C} \| \mathrm{D} & 0.25 & 1.42\end{array}$

$\begin{array}{lllll}138 & \mathrm{C} \mathrm{C} & \mid \mathrm{C} & 0.30 & 0.88\end{array}$

$\begin{array}{llll}107 & \text { B B ||B } 0.21 & 0.58\end{array}$

$\begin{array}{lllll}287 & D & C\end{array} \mid D \quad 0.32 \quad 1.56$

$\begin{array}{lllll}100 & \mathrm{C} & \mathrm{C} \| \mathrm{B} & 0.38 & 1.19\end{array}$

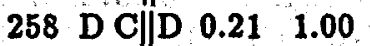

$\begin{array}{lll}1.29 & 0.56 & -26 .\end{array}$

$\begin{array}{llll}2.47 & 1.28 & -98 & \end{array}$

$4.53 \quad 3.80 \quad 26$.

$\begin{array}{llll}4.60 & 3.34 & -42 .\end{array}$

\begin{tabular}{lll}
6.35 & 6.33 & -103 \\
\hline
\end{tabular}

$\begin{array}{llll}3.46 & 1.72 & -117\end{array}$

$11.414 .63-116$.

$2.73 \quad 1.40-126$.

$3.512 .69-14$.

$\begin{array}{llll}7.36 & 5.40 & -66\end{array}$

$2.313 .22-126$.

$\begin{array}{lll}1.98 & 2.51 & -128\end{array}$

$\begin{array}{llll}0.76 & 2.02 & -57\end{array}$

$\begin{array}{llll}2.69 & 1.88 & -72\end{array}$

$\begin{array}{llll}0.94 & 1.38 & -97\end{array}$

$\begin{array}{llll}0.96 & 0.48 & -72 .\end{array}$

$\begin{array}{lll}1.21 & 1.45 & -79\end{array}$

$\begin{array}{lll}0.96 & 0.73 \quad 30 .\end{array}$

$7.49 \quad 10.12-107$

$\begin{array}{llll}0.74 & 0.51 & -54\end{array}$

$\begin{array}{llll}3.30 & 1.28 & -28\end{array}$

$\begin{array}{llll}1.85 & 0.72 & -15\end{array}$

$0.850 .65,-66$.

3.09 99.00 -106.

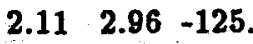

$4.01 \quad 6.02-111$.

DATE ORGIN LAT ${ }^{\circ}$ LONG $^{\circ} W$ DEP MAG MOD ST|PH DMIN GAP Q SQD RMS ERH1 ERH2 ERZ AZM

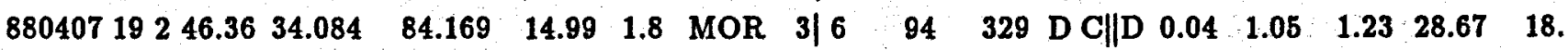
GA, DULUTH

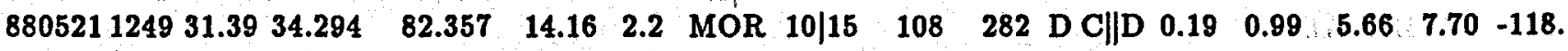
SC, SHOALS JUNCTION

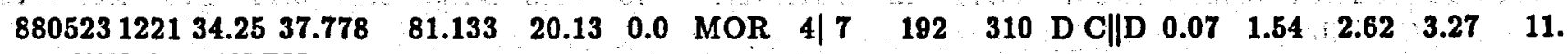
WV, BECKLEY

$8806169044.26 \quad 34.493$ GA, LYERLY

$88091816161.24 \quad 37.277$ KY, MILLPORT

881007222938.5438 .14 KY, OWINGSVILLE

88101391725.9434 .356

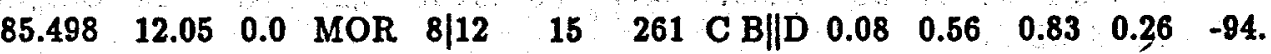

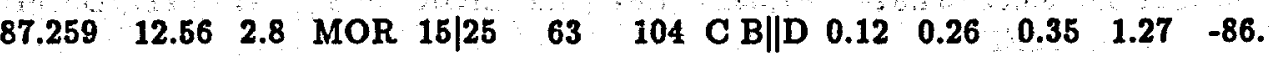

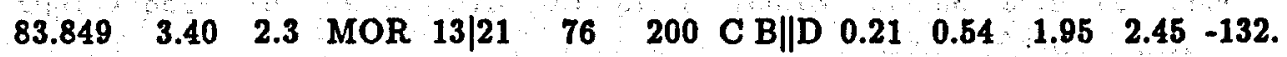

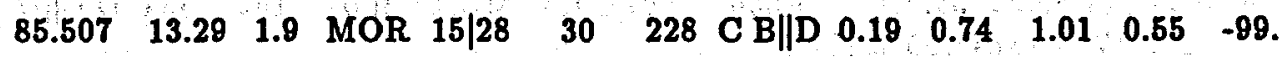

AL, GAYLESVILLE 


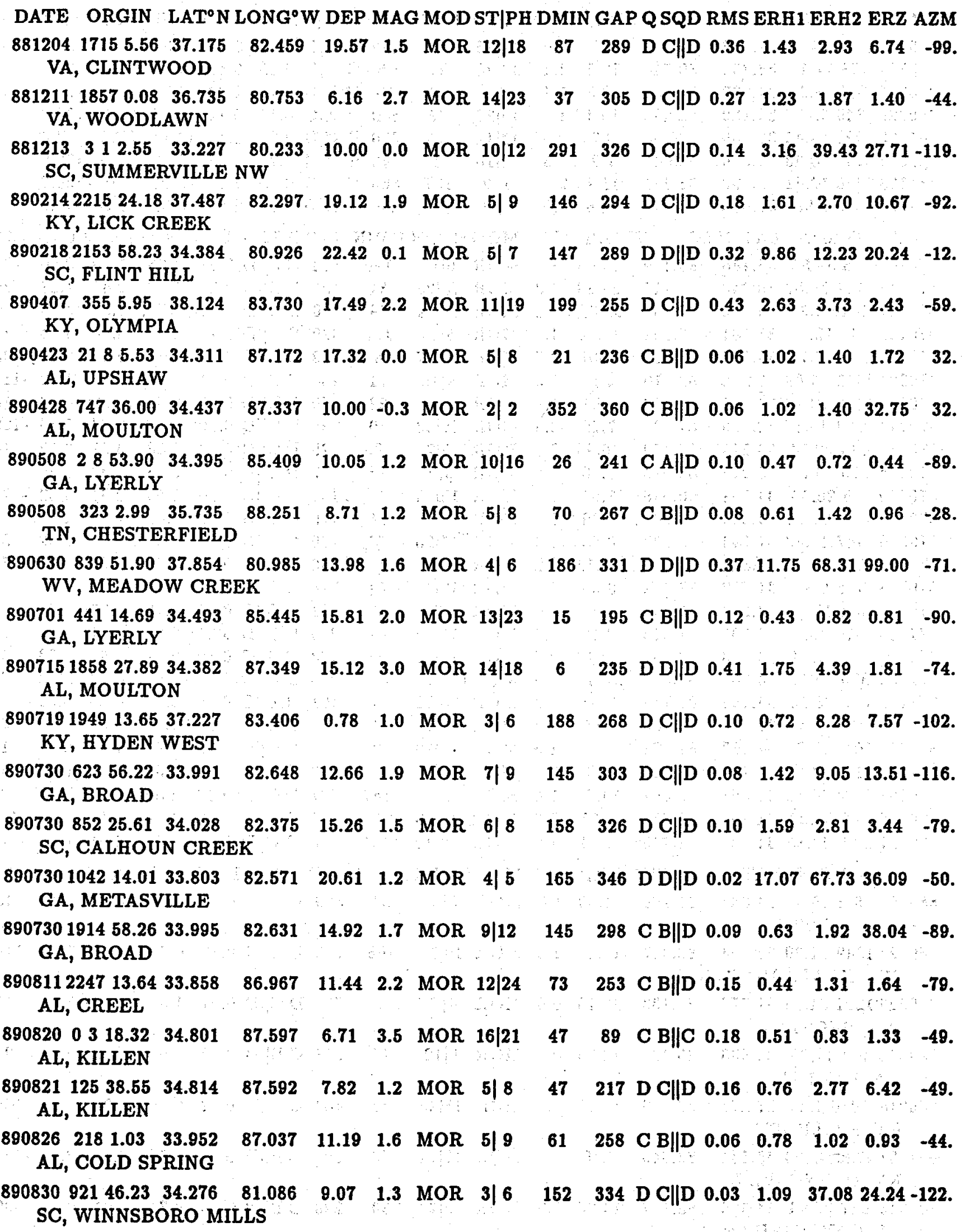


DATE ORGIN LAT $^{\circ} \mathrm{N}$ LONG ${ }^{\circ} \mathrm{W}$ DEP MAG MOD ST|PH DMIN GAP Q SQD RMS ERH 1 ERH2 ERZ AZM $\begin{array}{llllllllllllllllllllll}890831 & 543 & 12.21 & 35.747 & 80.488 & 4.21 & 1.1 & \text { MOR } & 5 / 8 & 69 & 283 & \text { C B BD } & 0.09 & 1.16 & 1.63 & 2.10 & 7 .\end{array}$ NC, SALISBURY

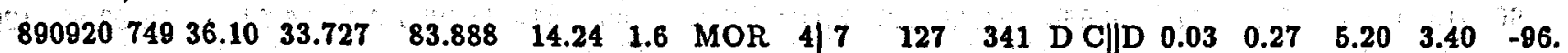
GA, MILSTEAD

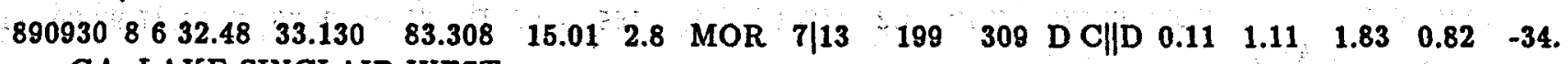
GA, LAKE SINCLAIR WEST

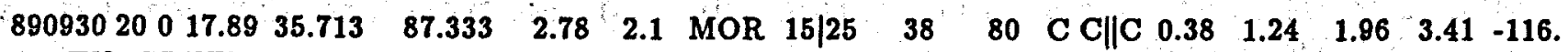
TN, GREENFIELD BEND

$89100811026.68 \quad 33.225 \quad 83.157$ GA, LAKE SINCLAIR EAST

$891010 \quad 112 \quad 27.78 \quad 33.224 \quad 83.218$ GA, LAKE SINCLAIR EAST

$89101174541.03 \quad 33.355 \quad 83.104$ GA, SHOULDERBONE

$90010342513.26 \quad 37.210 \quad 81.726$ VA, AMONATE

$90010312946.37 \quad 36.072 \quad 83.644$ TN, MASCOT

$900124182024.15 \quad 38.070 \quad 86.409$ KY, ALTON

$90012714549.31 \quad 38.108 \quad 86.430$ KY, ALTON

$900128 \quad 755 \quad 9.12 \quad 33.809$ GA, LINCOLNTON

$90012992325.28 \quad 34.339$ SC, POMARIA

900129 1941 44.49 38.332 IN, ENGLISH

$90020774139.92 \times 33.044$ SC, SUMMERVILLE $900227 \quad 31438.50 \quad 33.282$ GA, ROCKVILLE $90030921158.05 \times 38.049$ KY, ALTON

90032191137.6438 .146 KY, LOUISVILLE

90032617151.6334 .922 AL, CENTER HILL 000328101240.0034 .591 AL, OLD BETHEL 90040532936.4736 .714 VA, WOODLAWN

900408195223.0537 .281 KY, PARADISE

900410191838.4637 .801 KY, HAZEL GREEN

$\begin{array}{lllllllllllll}20.54 & 2.2 & \text { MOR } & 6 \mid 10 & 193 & 303 & D & D\end{array} \mid \begin{array}{lllllll}0.19 & 2.38 & 3.21 & 1.28 & -26 \text {. }\end{array}$

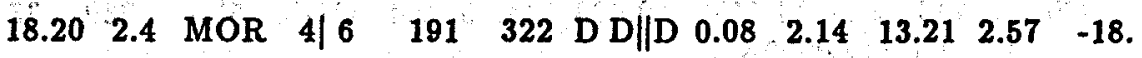
$\begin{array}{llllllllllllllll}15.98 & 2.0 & \text { MOR } & 6 \mid & 8 & 181 & 319 & \text { D C } & 0.15 & 0.23 & 3.14 & 3.47 & -51\end{array}$

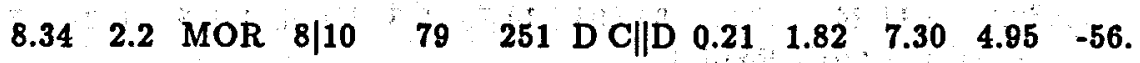

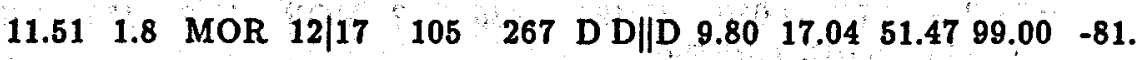

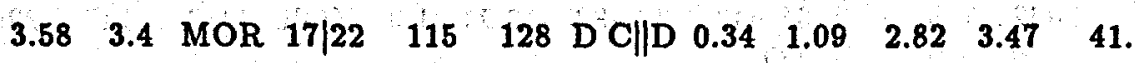
$\begin{array}{lllllllllllll}1.13 & 3.8 & \text { MOR } & 15 \mid 17 & 284 & 222 & \mathrm{D} & \mathrm{D}|| \mathrm{D} & 0.31 & 1.53 & 6.37 & 5.86 & -85\end{array}$ $\begin{array}{llllllllllllllllll}82.394 & 16.11 & -0.1 & \text { MOR } & 6 / 8 & 176 & 301 & \text { D C } & \text { D } & 0.12 & 1.59 & 2.07 & 2.59 & 41\end{array}$ $\begin{array}{lllllllllllllllll}81.481 & 14.94 & 1.3 \text { MOR } & 3 / 4 & 125 & 360 & \text { D D } & 0.05 & 12.32 & 17.0799 .00 & 7\end{array}$

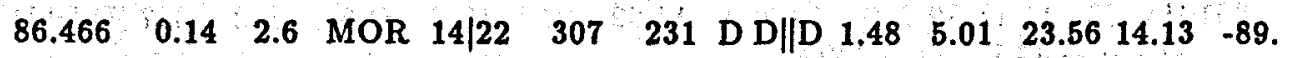
$80.227 \quad 17.942 .9$ MOR $4 / 6 \quad 309 \quad 338$ D D ||D $0.07 \quad 6.01 \quad 90.5159 .16-119$

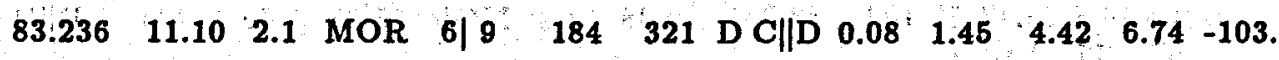

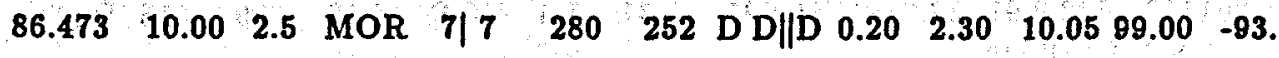
$\begin{array}{llllllll}85.818 \quad 5.76 & 2.4 & \text { MOR } & 3 / 5 & 256 & 328 & D & D\end{array} \mid$ D $0.04 \quad 29.05 \quad 51.4799 .00=123$.

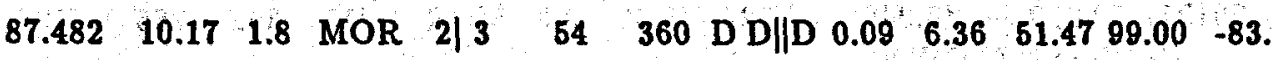

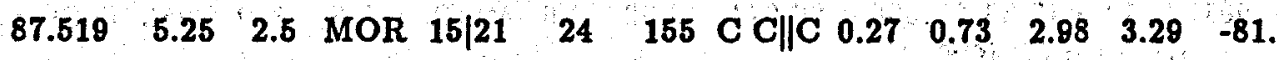

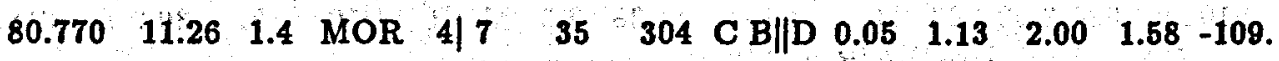
$\begin{array}{lllllllllllllllllllll}86.886 & 0.76 & 2.4 & \text { MOR } & 8 & 12 & 214 & 251 & D & C & \end{array}$

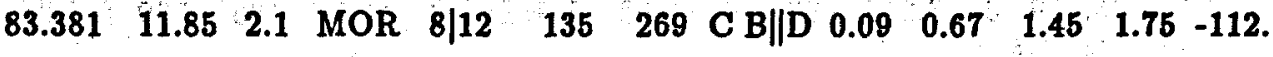


DATE ORGIN LAT ${ }^{\circ} \mathrm{N}$ LONG ${ }^{\circ} \mathrm{W}$ DEP MAG MOD ST|PH DMIN GAP Q SQD RMS ERH1 ERH2 ERZ AZM $\begin{array}{llllllllllllllllll}900504 & 2140 & 37.39 & 34.472 & 85.486 & 5.67 & 2.3 & \text { MOR } & 9 / 17 & 57 & 218 & \text { C B } & 0.11 & 0.58 & 1.67 & 2.07 & -49\end{array}$ GA, LYERLY

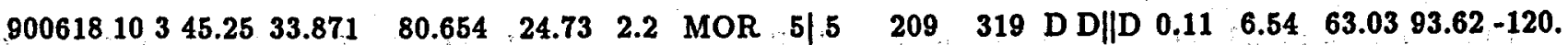
SC, WATEREE

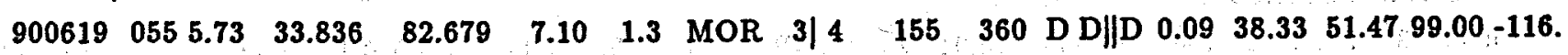
GA, TIGNALL

$\begin{array}{llllllllllllllllll}900623 & 2044 & 2.76 & 33.707 & 87.989 & 21.19 & 3.1 & \text { MOR } & 12 \mid 16 & 141 & 245 & \text { D C |D } & 0.25 & 1.60 & 2.33 & 1.35 & -54 .\end{array}$ AL, REGIONAL BLUFF

$\begin{array}{llllllllllllllllllll}900727 & 556.27 .47 & 34.402 & 85.489 & 6.50 & 2.2 & \text { MOR } & 6 / 10 & 65 & 215 & \text { C B } & \text { BD } & 0.11 & 1.12 & 1.43 & 1.35 & -49 .\end{array}$ GA, LYERLY

800818224652.3233 .643 SC, SUMMERTON

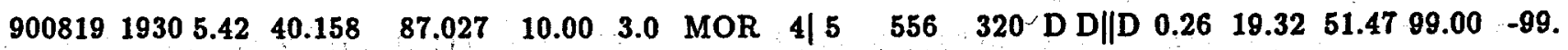
IL, WINGATE

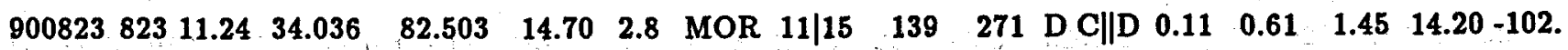
SC, CALHOUN FALLS

$\begin{array}{lllllllllllllllllllllll}900823 & 919 & 28.71 & 34.030 & 82.494 & 0.85 & 2.2 & \text { MOR } & 11 \mid 13 & 139 & 280 & \text { D C } & 0.12 & 0.89 & 5.74 & 5.23 & -110 .\end{array}$ SC, CALHOUN CREEK

$90082472545.16 \quad 33.948 \quad 82.482$ SC, WILLINGTON

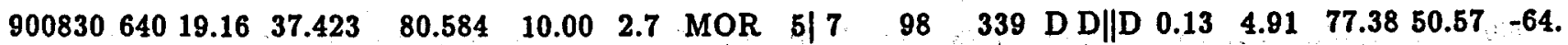
VA, INTERIOR

$90090243540.70 \quad 33.715 \quad 87.948$ AL, VERNON

$\begin{array}{llllllllllllllllllll}900926 & 1318 & 51.66 & 37.142 & 89.606 & 0.25 & 0.0 & \text { MOR } & 43 \mid 50 & 29 & 68 & \text { D D } & \text { |C } & 0.72 & 1.67 & 2.27 & 2.88 & 19 .\end{array}$ MO, SCOTT CITY

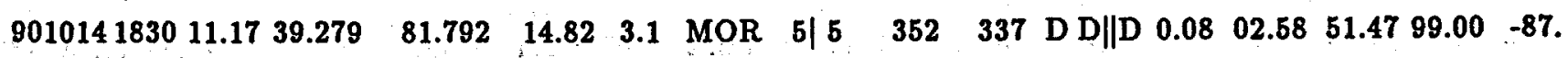
OH, LITTLE HOCKING

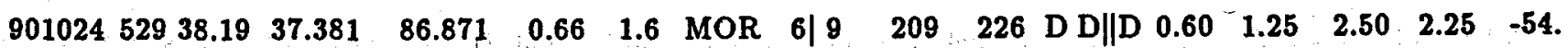
KY, HORTON

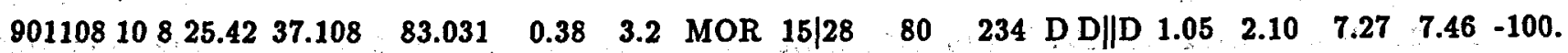
KY, TILFORD

$\begin{array}{llllllllllllllllll}901113 & 1522 & 9.91 & 32.934 & 80.021 & 9.31 & 3.5 & \text { MOR } & 14 \mid 16 & 329 & 316 & \text { D } & \text { D||D } & 0.27 & 5.49 & 47.75 & 33.58-123 .\end{array}$ SC, LADSON

$\begin{array}{lllllllllllllllllllll}901129 & 921 & 16.22 & 38.797 & 81.921 & 9.14 & 2.1 & \text { MOR } & 5 \mid 8 & 246 & 330 & \text { D } & \text { D||D } & 0.66 & 7.72 & 51.47 & 89.00 & -87 .\end{array}$ WV, MOUNT ALTO

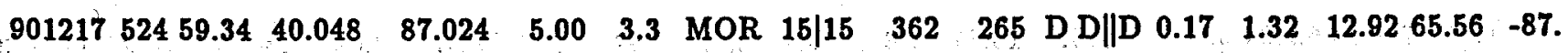
IN, WAYNETOWN

$\begin{array}{llllllllllllllllllllll}901220 & 14 & 4 & 17.36 & 39.596 & 86.671 & 0.53 & 3.6 & \text { MOR } & 15 \mid 15 & 340 & 255 & \text { D C } & \text { C } & 0.29 & 2.03 & 11.19 & 7.66 & -81 .\end{array}$ IN, EMINENCE

$\begin{array}{llllllllllllllllll}910108 & 624 & 8.68 & 33.220 & 83.194 & 14.38 & 2.5 & \text { MOR } & 11 \mid 19 & 192 & 302 & \text { D C } & \text { (|D } & 0.33 & 2.38 & 3.46 & 2.33 & -48 .\end{array}$ GA, LAKE SINCLAIR EAST

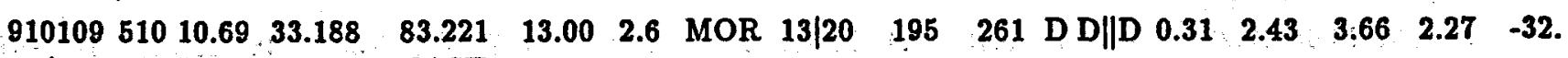
GA, LAKE SINCLAIR EAST

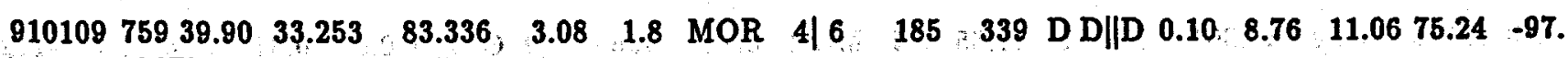
GA, MEDA 
DATE ORGIN LAT $^{\circ} \mathrm{N}$ LONG ${ }^{\circ} W$ DEP MAG MOD ST|PH DMIN GAP Q SQD RMS ERH1 ERH2 ERZ AZM

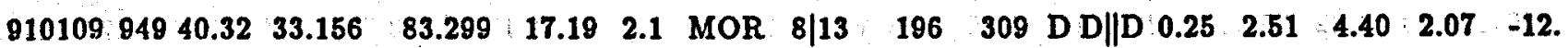
GA, LAKE SINCLAIR WEST

$910115848 \quad 22.48 \quad 33.204 \quad 83.205$

GA, LAKE SINCLAIR EAST

$910116152639.4033 .171 \quad 83.264$

GA, LAKE SINCLAIR WEST

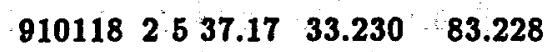

GA, LAKE SINCLAIR EAST

010121 T $323.72 \div 34.490 \quad 86.397$

AL, GUNTERSVILLE DAM

$91012632121.75 \quad 41.586 \quad 81.772$

OH, CLEVELAND NORTH

$91012722034.91 \quad 33.231 \quad 83.247$

GA, LAKE SINCLAIR EAST

$91020743 \quad 14.33 \quad 33.195 \quad 83.183$

GA, LAKE SINCLAIR EAST

$9102079127.7133 .192 \quad 83.141$

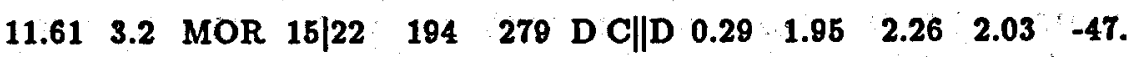

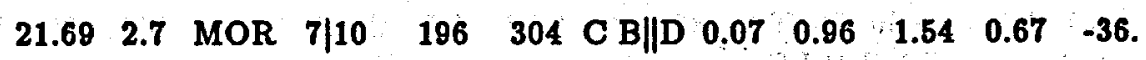

$\begin{array}{llllllllllllll}18.94 & 2.6 & \text { MOR } & 9 \mid 14 & 190 & 295 & \mathrm{D} & \mathrm{C}|| \mathrm{D} & 0.22 & 2.25 & 2.56 & 1.20 & -34\end{array}$

$\begin{array}{lllllllllllllllllllll}12.24 & 2.0 & \text { MOR } & 11 \mid 20 & 47 & 206 & \mathrm{C} & \mathrm{B} \| \mathrm{D} & 0.19 & 0.49 & 1.32 & 0.97 & -112 .\end{array}$

$\begin{array}{llllllllllll}10.00 & 3.4 & \text { MOR } & 11 \mid 12 & 549 & 336 & \text { D D } & 0.27 & 14.57 & 51.47 & 99.00 & -80\end{array}$

$\begin{array}{lllllllllllllllllll}20.35 & 3.1 & \text { MOR } & 8 / 12 & 190 & 295 & \mathrm{D} & \mathrm{C} \| \mathrm{D} & 0.18 & 2.12 & 3.23 & 1.23 & -37 \text {. }\end{array}$

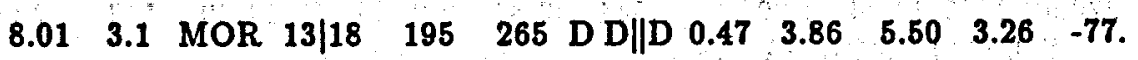

$\begin{array}{llllllllllllllll}19.65 & 2.0 & \text { MOR } & 7 \mid 12 & 197 & 304 & \text { D C } & 0.09 & 0.97 & 1.73 & 0.67 & -12 .\end{array}$

GA, LAKE SINCLAIR EAST

$910207 \quad 13150.67 \quad 33.197 \quad 83.181$

GA, LAKE SINCLAIR EAST

$\begin{array}{llllllllllllllllll}13.67 & 2.0 & \text { MOR } & 7 / 9 & 195 & 303 & \text { D C } & \text { |D } & 0.11 & 1.64 & 3.56 & 2.74 & -34\end{array}$

$910217173513.85 \quad 33.140 \quad 85.771$

AL, GIBSONVILLE

$\begin{array}{llll}910315 \quad 653 \quad 55.97 & 38.784 & 77.442\end{array}$

VA, MANASSAS

910402 $104134.5934 .439 \quad 84.082 \quad 14$

GA, DAWSONVILLE

$9104221119.27 \quad 37.996 \quad 80.266$

WV, ANTHONY

$010502103518.5734 .307 \quad 81.203$
SC RION

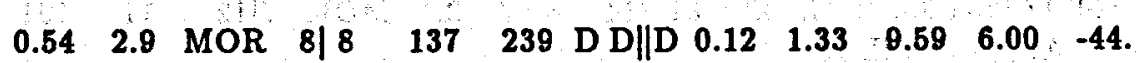

SC, RION

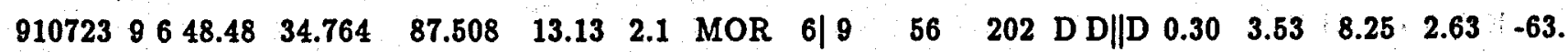

AL,KILLEN

$\begin{array}{lllllllllll}910809 & 135 & 29.18 & 33.981 & 82.507 & 14.99 & 0.7 & \text { MOR } & 6 \mid 8 & 145\end{array}$

GA, CHENNAULT. Possibly related to Clarks Hill Lake, SC

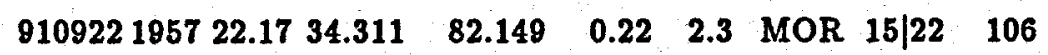

SC, COKESBURY. Possibly related to Greenwood Lake, SC

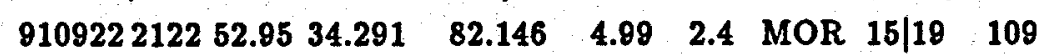

SC, COKESBURY. Possibly related to Greenwood Lake, SC

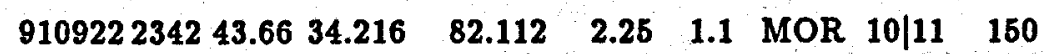

SC, NINETY SIX. Possibly related to Greenwood Lake, SC

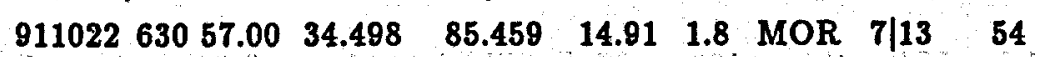

GA, LYERLY

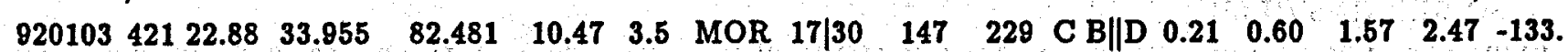

SC, WILLINGTON

$\begin{array}{llllllllllllllllllll}9201071148 & 29.32 & 33.412 & 82.783 & 2.23 & 2.7 & \text { MOR } & 9 \mid 10 & 188 & 290 & \text { D D } \mid \text { D } & 0.20 & 2.82 & 4.93 & 5.05 & -85 \text {. }\end{array}$ GA, SPARTA NE 
DATE ORGIN LAT $^{\circ} \mathrm{N}^{2} \mathrm{ONG}^{\circ} \mathrm{W}$ DEP MAG MOD ST|PH DMIN GAP Q SQD RMS ERH1 ERH2 ERZ AZM

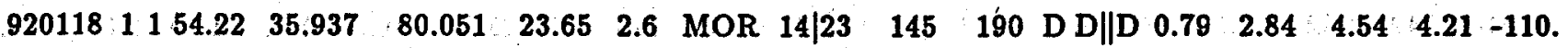
NC, HIGH POINT WEST

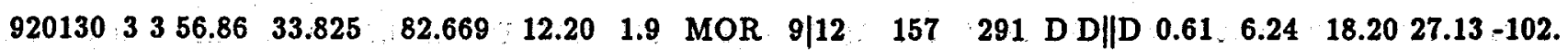
GA, TIGNALL

$\begin{array}{llllllllllllllllllllll}920130 & 312 & 55.06 & 33.766 & 82.593 & 8.77 & 1.5 & \text { MOR } & 5 & 6 & 166 & 307 & \text { D C } & \text { |D } & 0.17 & 2.18 & 21.08 & 37.23 & -102 .\end{array}$ GA, METASVILLE

$\begin{array}{lllllllllllllllllll}920130 & 657 & 6.98 & 34.057 & 82.557 & 14.99 & 1.8 & \text { MOR } & 9 \mid 11 & 147 & 269 & \text { D C C } & 0.16 & 1.39 & 3.84 & 11.45 & -91 .\end{array}$ SC, CALHOUN FALLS

$\begin{array}{lllllllllllllllllll}920201 & 44 & 54.69 & 33.742 & 82.365 & 4.14 & 1.7 & \text { MOR } & 6 \mid & 8 & 183 & 331 & \text { D C } & \text { C D } & 0.09 & 2.31 & 5.18 & 5.14 & -132 .\end{array}$

GA, LEAH

$\begin{array}{lllllllllllllllllllllll}920201 & 5 & 64.02 & 33.788 & 82.500 & 0.22 & 1.9 & \text { MOR } & 8 & 12 & 171 & 296 & \text { D D } & 0.34 & 4.00 & 24.07 & 25.74 & -118 .\end{array}$

GA, METASVILLE

92020184333.0233 .735 .

GA, WOODLAWN

920201141730.8634 .006 SC, CALHOUN FALLS

$920209 \quad 136 \quad 22.70 \quad 33.725$

GA, LEAH

$92022962918.15 \quad 35.563$

TN, COLUMBIA

920317173135.2234 .432

AL, OAKVILLE

$9203225723.12 \quad 35.049$

TN, ST JOSEPH

$920405 \quad 55432.59 \quad 34.635$

AL, TOWN CREEK

920405115736.9634 .627

AL, TOWN CREEK

$92042675452.61 \quad 32.725$

AL, MELTON

92051164229.8436 .840

KY, MILBURN

$\begin{array}{lllll}920519 & 845 & 6.18 & 36.771\end{array}$

VA, HILLSVILLE

920616192848.7538 .061 IN, ALTON

920802103621.0034 .245 GA, BIRMINGHAM

$920821 \quad 16322.82 \quad 33.656$ SC, ELLOREE

$9209294522.99 \quad 37.286$ WV, BRADSHAW

92102952454.4134 .364 SC, JENKINSVILLE

$92112422651.25 \quad 37.439$ WV, LERONA

$\begin{array}{lllllllllllllllll}82.447 & 10.43 & 2.5 & \text { MOR } & 18 & 26 & 171 & 278 & \text { D C } & \text { D } & 0.42 & 2.37 & 3.40 & 3.93 & -127 .\end{array}$

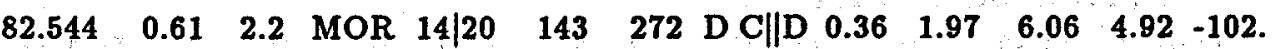

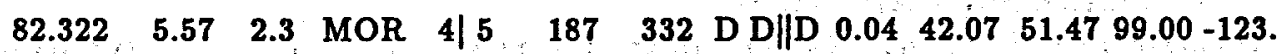

$\begin{array}{llllllllllllllll}87.091 & 14.49 & 2.3 & \text { MOR } & 15 \mid 27 & 64 & 86 & \text { D C } & 0.38 & 0.38 & 0.74 & 1.13 & 3.76 & -37 .\end{array}$

$\begin{array}{lllllllllllllllll}87.214 & 8.97 & 2.1 & \text { MOR } & 7 \mid 12 & 11 & 191 & \text { D D } & 0.32 & 0.16 & 3.26 & 2.04 & -103\end{array}$

$\begin{array}{lllllllllllllllllll}87.597 & 0.01 & 2.0 & \text { MOR } & 4 / 7 & 43 & 158 & \text { D D } & \text { IIC } & 8.72 & 16.05 & 39.53 & 80.53 & -9\end{array}$

$\begin{array}{lllllllllllllll}87.421 & 15.29 & 2.1 & \text { MOR } & 7 \mid 12 & 23 & 186 & \text { D C }|| D & 0.21 & 1.21 & 2.72 & 8.48 & -65 \text {. }\end{array}$

$\begin{array}{lllllllllllllll}87.458 & 19.03 & 2.0 & \text { MOR } & 9 \mid 14 & 24 & 153 & \text { C C } & \text { C } & 0.24 & 1.20 & 1.69 & 3.24 & -89\end{array}$

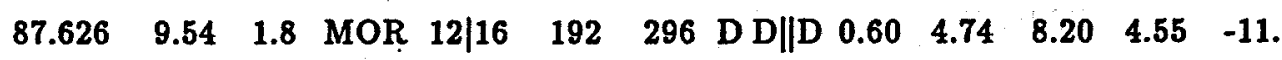

$\begin{array}{llllllllllllll}88.996 & 5.34 & 2.3 & \text { MOR } & 14 \mid 17 & 85 & 202 & \text { D C } & \text { C D } & 0.34 & 1.46 & 2.73 & 3.56 & -109 \text {. }\end{array}$

$\begin{array}{llllllllllllll}80.735 & 11.26 & 2.4 & \text { MOR } & 15 \mid 23 & 40 & 176 & \text { C C } & \text { C C } & 0.37 & 0.89 & 1.99 & 2.45 & 41 .\end{array}$

$\begin{array}{llllllllllllllll}87.464 & 5.47 & 2.8 & \text { MOR } & 9 \mid 16 & 22 & 235 & D & C & 0 & 0.29 & 1.05 & 2.66 & 4.03 & 6\end{array}$

\begin{tabular}{lllll|lllllllllll}
84.322 & 0.70 & 1.7 & MOR & 5 & 6 & 76 & 360 & D D & 0.19 & 2.96 & 20.51 & 22.56 & -64
\end{tabular}

$\begin{array}{llllllllllllll}80.508 & 7.50 & 3.8 & \text { MOR } & 13 \mid 13 & 240 & 305 & \text { D D } & \text { D } & 0.12 & 1.90 & 17.66 & 7.86 & -121\end{array}$

$\begin{array}{lllllllllllllllllll}81.810 & 8.51 & 3.2 & \text { MOR } & 7 \mid 8 & 90 & 254 & D & C & \| D & 0.12 & 1.16 & 4.85 & 6.83 & -84\end{array}$

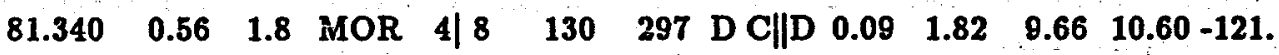

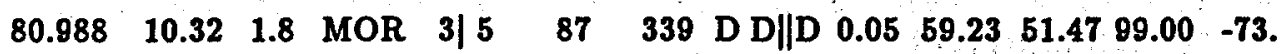


Southern Appalachian Regional Seismic Network

Shu-Chioung Chi Chiu, Arch Johnston, Jer-Ming Chiu

August 1994

4. FIN OR GRANT NUMBER

D1553

6. TYPE OF REPORT

\section{Technical}

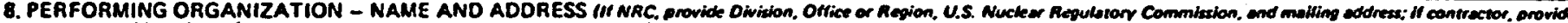
ame and ariling eddress:

Memphis State University

Center for Earthquake Research and Information

Memphis, Tennessee 38152

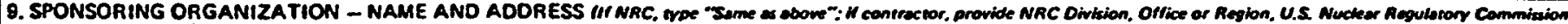
and meiting eddrese)

Division of Engineering

Office of Nuclear Regulatory Research

U.S. Nuclear Regulatory Commission

Washington, D.C. 20555-0001

10. SUPPLEMENTARY NOTES

\section{ABSTRACT 1200 monds or kasl}

Memphis State University has monitored the seismicity of the southern

Appalachian area since late 1979 by means of the Southern Appalachian Regional

Seismic Network (SARSN), which has provided good spatial coverage for

earthquake location. Activity is more heavily concentrated in the Valley and

Ridge province (VR) of eastern Tennessee than in the Blue Ridge (BR) or

Piedmont (P). The majority of these events 1 ie between the New York-Alabama and the Clingman/0coee lineaments, magnetic anomalies of deep-seated basement structures. Thus, SARSN has been able to define the first order characteristics of the Southern Appalachian seismic zone. The focal depths of the earthquakes are concentrated between 8 and $16 \mathrm{~km}$, principally beneath the Appalachian overthrust. In cross-section the average seismicity is shallower beneath the BR and P provinces than the VR and North American craton. Results of focal mechanism studies for events that occurred between October 1986 and December 1991, indicate that the basement of the VR province is under horizontal, NE-SW compressive stress. Right-lateral strike-slip faulting on nearly $\mathrm{N}-\mathrm{S}$ faults is preferred as it agrees with the trend of the regional magnetic anomaly pattern.

Seismicity

Eastern Tennessee Seismic Zone

Southern Appalachian Seismic Zone

Eastern U.S. Seismicity

Seismic Monitoring

Southern Appalachian Regional Seismic Network unl imited Ba. secualtr CuAssification Tratsoos unclassified rma hepond unclassified 15. NUMBER OF PAGES 


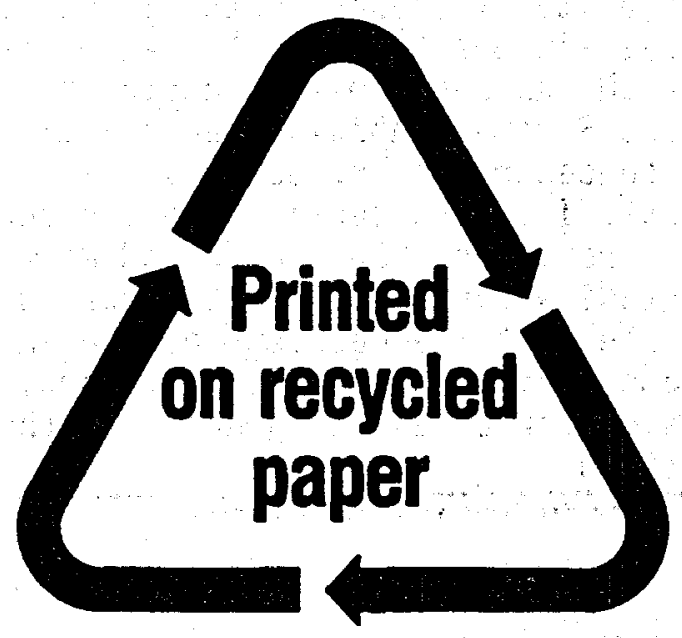

Federal Recycling Program 